Aus der Klinik für Neurochirurgie

(Prof. Dr. med. V. Rohde)

der Medizinischen Fakultät der Universität Göttingen

\title{
Operationsbedürftige abdominelle Shunt- Komplikationen beim kindlichen Hydrozephalus und der Stellenwert einer interdisziplinären chirurgischen Versorgung
}

\author{
INAUGURAL-DISSERTATION \\ zur Erlangung des Doktorgrades \\ der Medizinischen Fakultät der \\ Georg-August-Universität zu Göttingen
}

vorgelegt von

Georg Hoene

aus

Rostock

Göttingen 2020 
Dekan:

\section{Betreuungsausschuss}

Betreuer:

Ko-Betreuer:

Mitbetreuung:

\section{Prüfungskommission}

Referent:

Ko-Referent:

Drittreferent:

Datum der mündlichen Prüfung:
Prof. Dr. W. Brück

Prof. Dr. med. H. C. Ludwig

PD Dr. med. M. Grade

Dr. med. F. Kahl und Dr. med. H. Bock
Prof. Dr. med. H. C. Ludwig

PD Dr. med. M. Grade

Prof. Dr. T. Meyer

7. Mai 2020 
Hiermit erkläre ich, die Dissertation mit dem Titel: "Operationsbedürftige abdominelle Shunt-Komplikationen beim kindlichen Hydrozephalus und der Stellenwert einer interdisziplinären chirurgischen Versorgung" eigenständig angefertigt und keine anderen als die von mir angegebenen Quellen und Hilfsmittel verwendet zu haben.

Göttingen, den 


\section{Inhaltsverzeichnis}

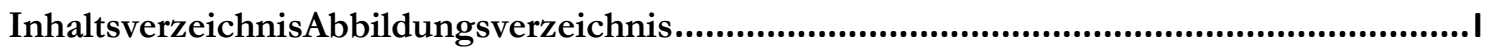

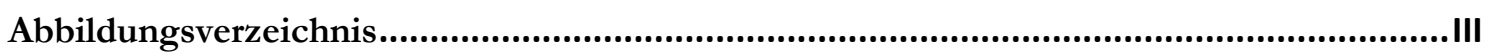

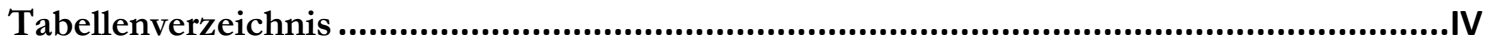

Abkürzungsverzeichnis.......................................................................................................

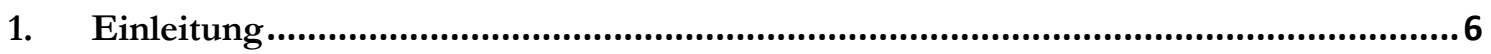

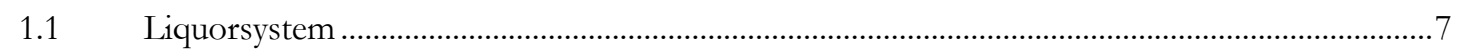

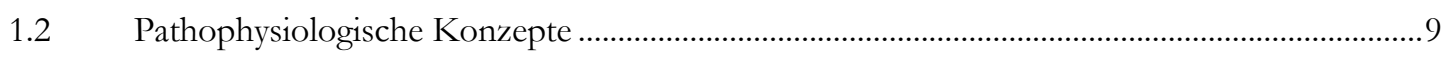

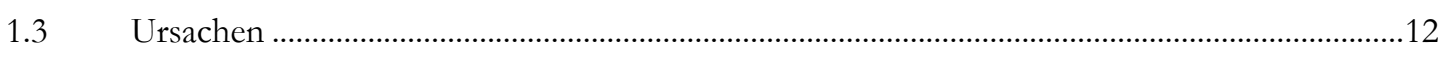

1.4 Posthämorrhagischer Hydrozephalus und intraventrikuläre Hirnblutung ................................14

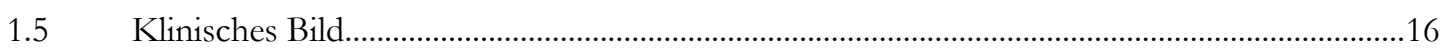

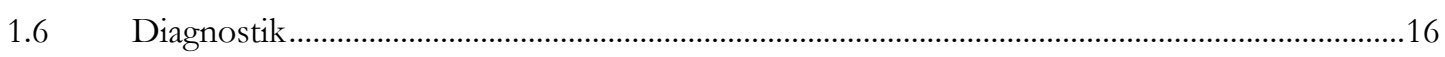

1.7 Therapieoptionen des kindlichen Hydrozephalus .......................................................................

1.7.1 Pharmakologische Therapieoptionen .......................................................................................17

1.7.2 Neuroendoskopie: ETV (Endoscopic Third Ventriculostomy).................................................18

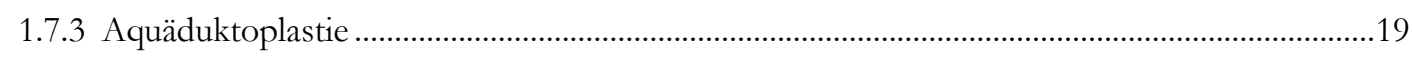

1.7.4 Plexus-Choroideus-Kauterisation .....................................................................................20

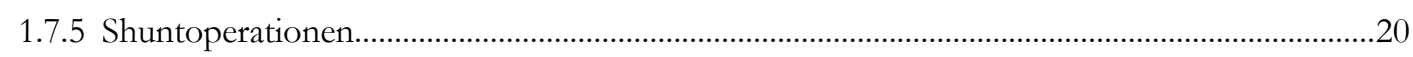

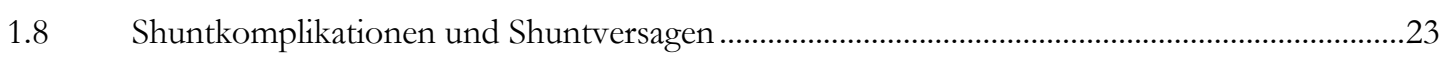

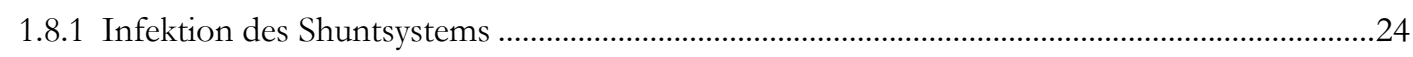

1.8.2 Mechanische und physikalische Probleme................................................................................26

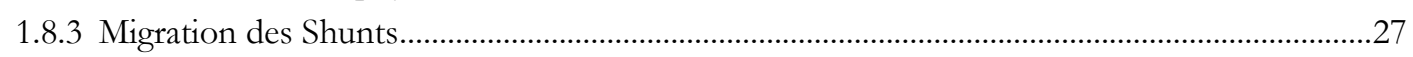

1.8.4 Überdrainage und Slit-Ventrikel-Syndrom ..........................................................................2

1.8.5 Nachträgliche Kompartimentierung des Liquorraums ............................................................29

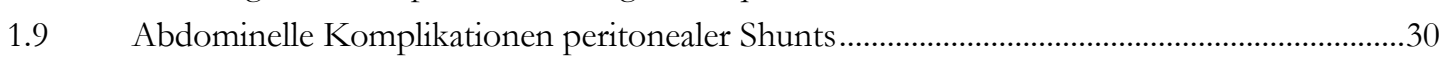

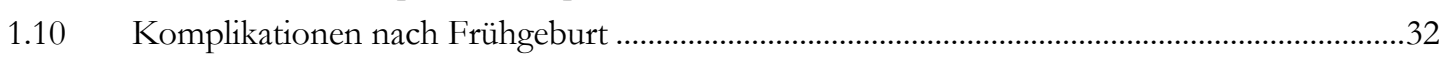

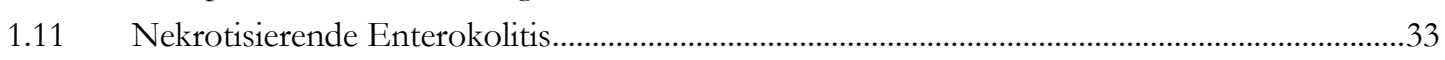

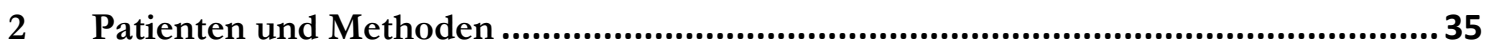

$2.1 \quad$ Versorgung von Kindern mit Hydrozephalus an der UMG.....................................................35

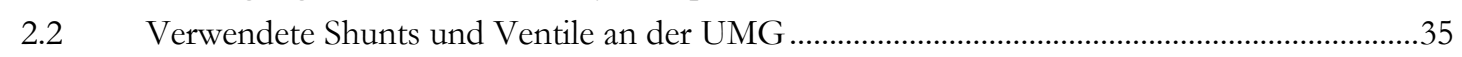

$2.3 \quad$ VPS-Neuanlage - Standardprozedere an der UMG ………………………………………….........

2.4 Datenerfassung und Selektion des Patientenkollektivs..............................................................38

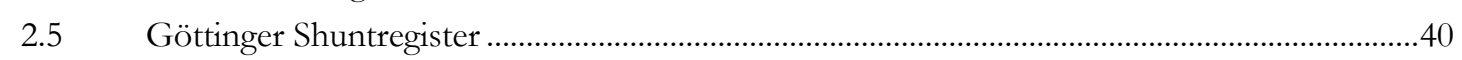

2.6 Erfasste Parameter der Datenauswertung …………………………………………...................4

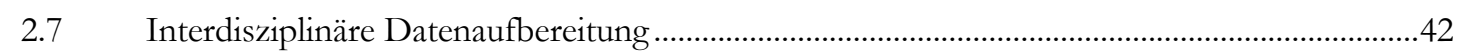

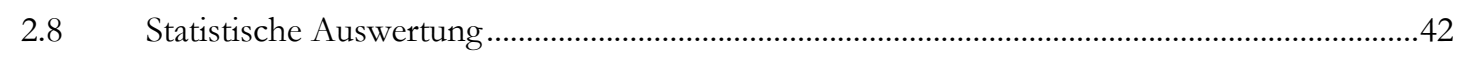

$2.9 \quad$ Datenschutzkriterien und Literaturrecherche ………………………………………...................43

3 Ergebnisse

3.1 Darstellung des Patientenkollektivs …………………………………………………….....4

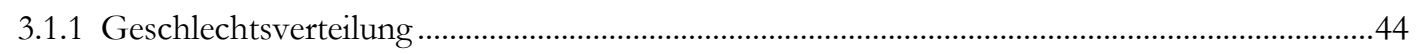

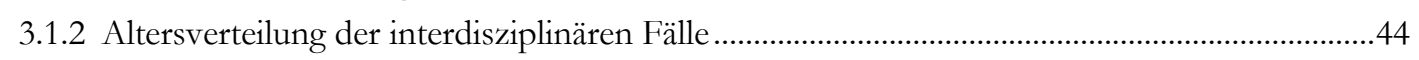


3.1.3 Anzahl der gesamten Shuntrevisionen pro Patient .................................................................45

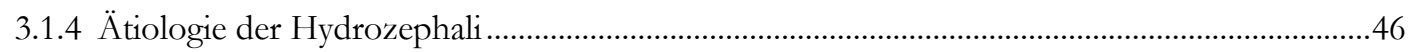

3.2 Potentielle Risikofaktoren für abdominelle Shuntkomplikationen ..........................................47

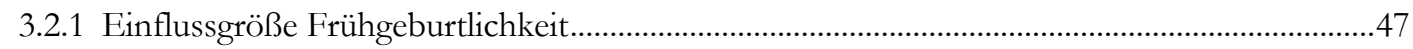

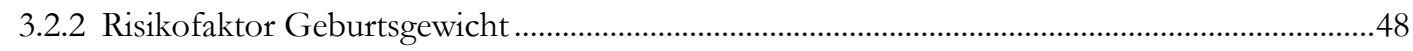

3.2.3 Untersuchung zum Einteilungsgrad der intraventrikulären Blutungen....................................49

3.2.4 Frühgeborenenstatus und nekrotisierende Enterokolitis ......................................................50

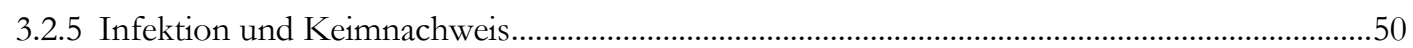

3.3 Indikationsstellung zur interdisziplinären Shuntrevision ..........................................................51

3.3.1 Übersicht der Operationszugänge zum distalen Teil des VPS ...............................................51

3.3.2 Ableitungsart nach interdisziplinärer Versorgung ......................................................................52

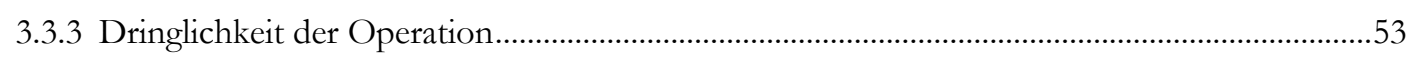

3.3.4 Operationsindikation aus neurochirurgischer Sicht ...............................................................54

3.3.5 Operationsindikation aus kinderchirurgischer Sicht .................................................................56

3.4 Analyse des Zeitraums nach interdisziplinärer Versorgung........................................................58

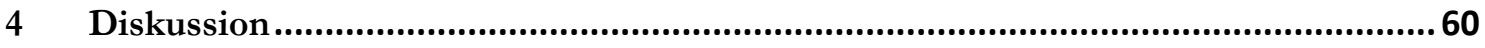

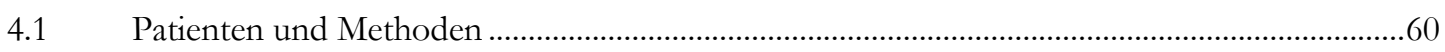

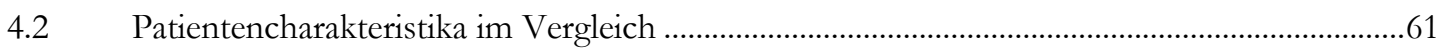

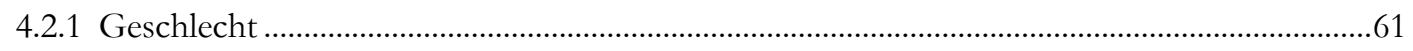

4.2.2 Alter zum Zeitpunkt der Versorgung.........................................................................................61

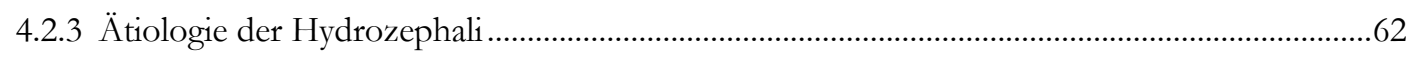

4.2.4 Anzahl der Shuntrevisionen ....................................................................................................64

4.3 Risikofaktoren für abdominelle Shuntkomplikationen ..........................................................65

4.3.1 Posthämorrhagischer Hydrozephalus in Bezug zum Blutungsgrad nach Papile ......................65

4.3.2 Frühgeburtlichkeit als Risikofaktor für abdominelle Komplikationen nach ventrikulo-

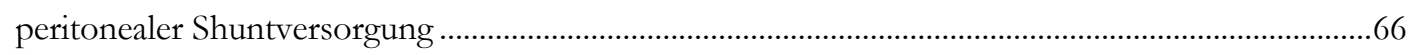

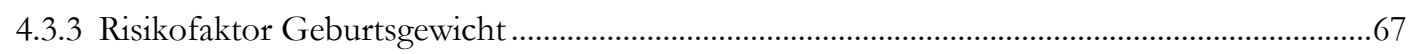

4.3.4 Nekrotisierende Enterokolitis als Risikofaktor für abdominelle Kompli-kationen nach

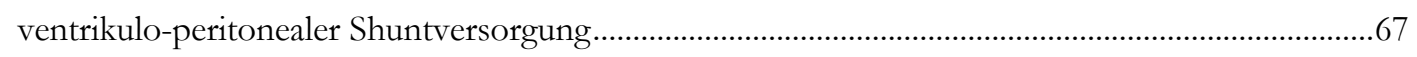

4.3.5 Infektionsnachweis bei abdominellen Komplikationen ...........................................................68

4.4 Interdisziplinäre Versorgung abdomineller Shuntkomplikationen ............................................69

4.4.1 Operationszeitpunkt, Operationszugänge und Ableitungsart nach chirurg-ischer Versorgung

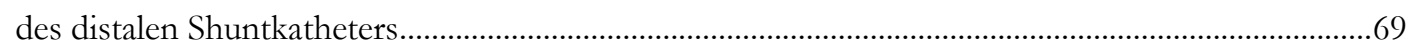

4.4.2 Kinderchirurgische abdominelle Komplikationen ..................................................................72

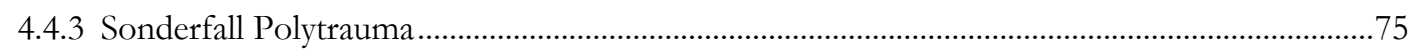

4.4.4 Neurochirurgische Operationsindikationen der Fälle..................................................................75

4.4.5 Postoperativer Verlauf und Follow-up.........................................................................................78

4.5 Vorteile und Nutzen einer interdisziplinären Versorgung .......................................................79

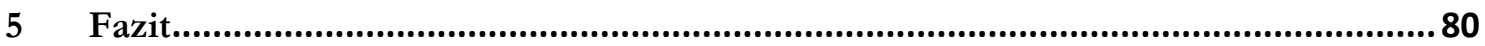

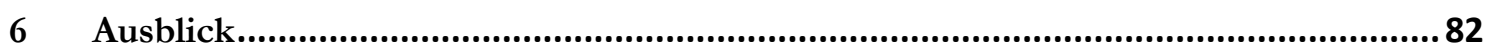

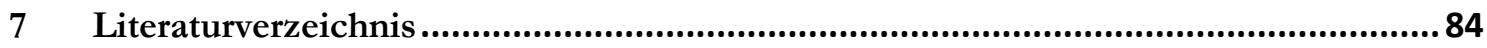




\section{Abbildungsverzeichnis}

Abbildung 1: Auswahl des Patientenkollektivs. .38

Abbildung 2: Alter zum Zeitpunkt der interdisziplinären Versorgung in Jahren . .44

Abbildung 3: Ursachen des kindlichen Hydrozephalus im Überblick . .46

Abbildung 4: Einteilung des Fallkollektivs nach Schwangerschaftswoche .............................................47

Abbildung 5: Darstellung des Blutungsgrads nach Lou Ann Papile .......................................................49

Abbildung 6: Einteilung des operativen Zugangswegs zum distalen Katheter .....................................51

Abbildung 7: Ableitungsart des Shunts postinterventionell ....................................................................52

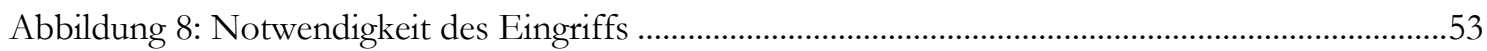

Abbildung 9: Neurochirurgische Operationsindikationen …………………………………………......5

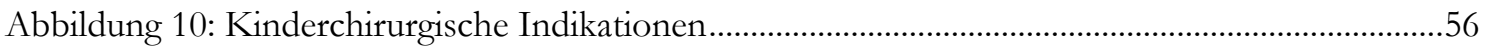

Abbildung 11: Zeitraum nach interdisziplinärer Behandlung ...........................................................58

Abbildung 12: Ventrikulo-peritonealer Shunt in nicht infizierter Liquorpseudozyste..........................73 


\section{Tabellenverzeichnis}

Tabelle 1: Ursachen des kindlichen Hydrozephalus

Tabelle 2: Vergleich Klassifikationen intrakranieller Blutungen bei Frühgeborenen

(Papile/DEGUM)

Tabelle 3: Distale historische Ableitungsoptionen zur Liquorresorption ..........................................21

Tabelle 4: Studienübersicht zur Infektionsrate und Inzidenz bei VP-Shunts....................................24

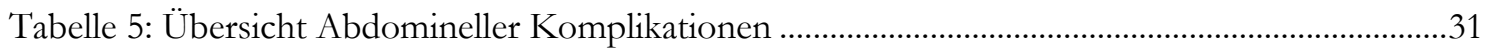

Tabelle 6: Kurz und langfristige Probleme bei Frühgeborenen.........................................................32

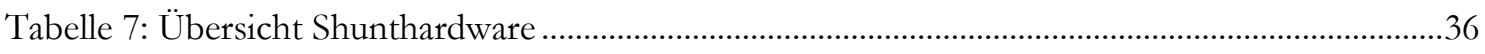

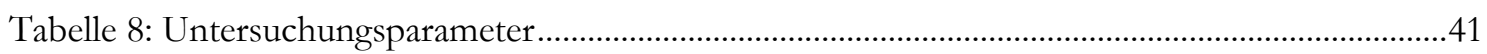

Tabelle 9: Deskriptive Statistik der gesamten Shuntrevisionen pro Patient .......................................45

Tabelle 10: Deskriptive Statistik der Frühgeburtlichkeit in SSW .......................................................48

Tabelle 11: Deskriptive Statistik des Geburtsgewichts der Frühchen ...............................................48

Tabelle 12: Kreuztabelle Frühgeborenenstatus * NEC...................................................................50

Tabelle 13: Kreuztabelle zur Darstellung von Infektion und Keimnachweis.......................................50

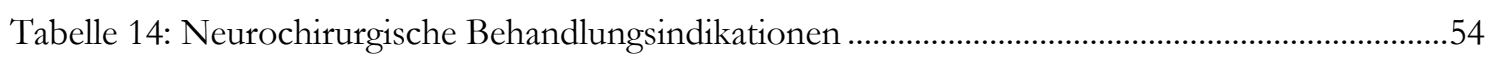

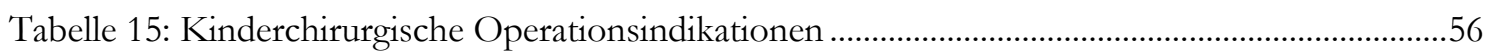

Tabelle 16: Deskriptive Statistik des Zeitraums nach interdisziplinärer Versorgung...........................58

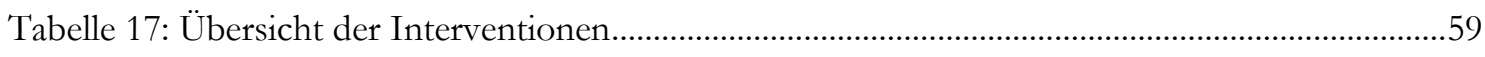

Tabelle 18: Vergleich der Ätiologieverteilung verschiedener Studien.................................................63

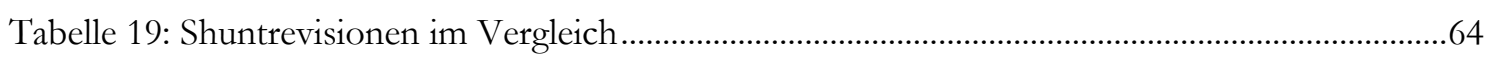

Tabelle 20: Infektionsraten von abdominellen Liquorzysten in verschiedenen Fallstudien ...............73 


\section{Abkürzungsverzeichnis}

CPC eng. choroid plexus cauterization - dt. Plexus-Choroideus-Kauterisation

DD Differentialdiagnose

ETV eng. endoscopic third ventriculostomy - dt. Endoskopische Drittventrikulostomie

EVD externe Ventrikeldrainage

HC Hydrozephalus

ICP intrakranieller Druck

IRDS eng. infant respiratory distress syndrome - dt. Atemnotsyndrom des Neugeborenen

NEC nekrotisierende Enterokolitis

PHHC posthämorhagischer Hydrozephalus

ROP eng. retinopatby of prematurity - dt. Frühgeborenen-Retinopathie

SSW Schwangerschaftswoche

SVS eng. slit ventricle syndrom - dt. Schlitz-Ventrikel-Syndrom

VA ventrikulo-atrialer Shunt

VP/VPS ventrikulo-peritonealer Shunt

Z. n. Zustand nach 


\section{Einleitung}

Interdisziplinäre Zusammenarbeit gilt im Rahmen der heutigen medizinischen Versorgung als elementare Voraussetzung für eine effektive, erfolgreiche und ganzheitliche Patientenversorgung. Durch die kontinuierlichen Weiterentwicklungen und Spezifizierungen medizinischer Fachdisziplinen werden die Anforderungen an das Fachpersonal immer komplexer und machen die Notwendigkeit fachübergreifender Kommunikation und Kooperation deutlich. Bei dem sehr heterogenen Krankheitsbild des kindlichen Hydrozephalus beinhaltet dies die Zusammenarbeit von Neuropädiatern, Neurochirurgen, Kinderchirurgen, Pflege, Sozialarbeitern und weiteren Fachbereichen.

Vor diesem Hintergrund beleuchtet die vorliegende Dissertation Erfolgs- und Risikofaktoren sowie die Grenzen interdisziplinärer Zusammenarbeit im Kontext der Behandlung des kindlichen Hydrozephalus. Das Hauptaugenmerk liegt hierbei insbesondere auf möglichen abdominellen Komplikationen nach ventrikulo-peritonealer Shuntversorgung, weil sich an dieser Stelle ein anatomisches und chirurgisches Gebiet zweier Disziplinen trifft.

Grundlage bildet die Untersuchung konkreter Behandlungsmethoden an der Universitätsmedizin Göttingen mit dem Fokus auf der interdisziplinären Versorgung durch die Klinik für Allgemein-Viszeral/Kinderchirurgie und Klinik für Neurochirurgie. Hierfür wurden aus einem institutionellen Gesamtkollektiv alle Fallakten mit abdominellen Shuntkomplikationen selektiert, die eine interdisziplinäre Versorgung erforderlich machten. In diesem Zusammenhang werden hier die Vorgehensweisen pädiatrischer Shuntversorgung analysiert, die damit verbundenen Schnittstellen beider chirurgischen Disziplinen dargestellt und vor dem Hintergrund der dieser Arbeit zugrunde liegenden Fragestellung untersucht, ob unter bestimmten Voraussetzungen standardmäßig vor Beginn der Behandlung eine interdisziplinäre Kommunikation und Kooperation zwischen behandelnden Ärzten der Kinderchirurgie und Neurochirurgie die Operationsdurchführung und das Operationsergebnis positiv beeinflussen kann. 


\subsection{Liquorsystem}

Hirngewebe, Blut und Liquor bilden gemäß der Monro-Kellie-Doktrin die drei Hauptbestandteile des Gehirns. Schädelknochen, welche dieses wie eine feste Hülle umgeben, verhindern eine beliebige Ausdehnung der drei oben genannten Komponenten, die somit in ihren Volumina nach der Monro-Kellie Doktrin weitgehend konstant sind (Monro 1783; Kellie 1824). Der Liquor cerebrospinalis ist eine klare, wasserähnliche Flüssigkeit, deren Zusammensetzung im Grunde der des Blutplasmas entspricht. Das Volumen des Liquors beträgt beim Erwachsenen etwa insgesamt $150 \mathrm{ml}$, davon $50 \%$ intrakraniell und 50\% intraspinal, von denen sich ungefähr ein Viertel in den vier Ventrikeln und drei Viertel im Subarachnoidalraum befinden. Durchschnittlich werden pro Stunde $20 \mathrm{ml}$ Liquor produziert, wobei diese Werte tageszeitlichen Schwankungen unterliegen und es beispielsweise während der Nacht und am Morgen zu einer verstärkten Produktion kommt.

Ein Neugeborenes besitzt eine Gesamtliquormenge von wenigen Millilitern. Die Liquorbildung steigert sich dann mit zunehmendem Alter und entspricht bei einem Zehnjährigen mit einer Menge von $120 \mathrm{ml}-140 \mathrm{ml}$ bereits der Untergrenze eines Erwachsenen (Stauffer et al. 1992; Kirsch et al. 2011). Die Liquormenge folgt naturgemäß dem Hirnvolumen.

Ausgehend von den Seitenventrikeln zirkuliert der Liquor durch eine halbmondförmige Öffnung zwischen Fornix und vorderem Thalamus, den Foramina Monroi in den dritten Ventrikel (Kirsch et al. 2011, S. 832) und von dort aus weiter durch den Aquaeductus cerebri in den vierten Ventrikel. Diesen Zusammenschluss von Ventrikeln und Aquädukt bezeichnet man als inneren Liquorraum. Der äußere Liquorraum (Subarachnoidalraum) steht über die Aperturae laterales (Foramina Luschkae) und die Apertura mediana (Foramen Magendii) mit dem inneren Raum in Kontakt. Schließlich setzt sich das Ventrikelsystem in den Canalis Centralis im Rückenmark verlaufend bis in Höhe des Conus medullaris in Höhe des ersten Lendenwirbelkörpers fort (Trepel 2015).

Ein Großteil der wissenschaftlichen Forschungsergebnisse in dieser Thematik basieren auf den Untersuchungen und Tierversuchen zur Anatomie des Gehirn von Walter Edward Dandy (Horwitz 1997) und folgen den Annahmen, dass die Resorption des Liquor im äußeren Liquorraum über die sog. Arachnoidalzotten erfolgt, welche Ausstülpungen der Arachnoidea mater sind und in diesem Bereich in die abführenden venösen Gefäße der harten Hirnhaut des Gehirns einstrahlen (Segal 1993; Trepel 2015). Hiernach sind an der 
Wiederaufnahme des Liquors insbesondere die Perineuralscheiden der aus dem Rückenmark austretenden Spinal- und Hirnnerven beteiligt (Kirsch et al. 2011). Neuere Erkenntnisse in diesem Bereich, welchen diese Dissertation in den nachfolgenden Ausführungen folgen wird, stellen die oben beschriebene und in der Wissenschaft nach wie vor dominierende Meinung jedoch in Frage und gehen vielmehr davon aus, dass die Liquorproduktion und Resorption im Hirn- und Rückenmarksparenchym erfolgt und das Ventrikelsystem der simultanen Kommunikation und Liquordynamik. dient. Auf diese Weise herrscht ein Fließgleichgewicht, das vor allem durch osmotische und hydrostatische Drücke getragen ist. Die kleinen Gefäße des Gehirnparenchyms sind hierbei maßgeblich beteiligt (Orešković und Klarica 2010).

Der Liquordruck des liegenden Patienten fluktuiert zwischen 60-200 $\mathrm{mm} \mathrm{H}_{2} \mathrm{O}$. Durch Atmung und Puls sowie Bauchpresse können rhythmische Schwankungen von 3-4 mm $\mathrm{m}_{2} \mathrm{O}$ bei Kindern bis zum ersten Lebensjahr und bis zu $15 \mathrm{~mm} \mathrm{H}_{2} \mathrm{O}$ bei Erwachsenen hervorgerufen werden, die jedoch keinen Krankheitswert besitzen. In einem gewissen Maße können diese kurzzeitigen intrakraniellen Druckerhöhungen durch Volumenverschiebung des Liquor aus dem Schädel in den elastischen Spinalkanal kompensiert werden (Sakka et al. 2011). Die Gehirn- und Rückenmarksflüssigkeit hat vor allem eine mechanische Schutzfunktion. Durch Auftrieb wird das effektive Gewicht von ca. $1500 \mathrm{~g}$ auf $50 \mathrm{~g}$ reduziert. Zudem können durch die Trägheit des Gehirns im flüssigen Medium Erschütterungen effektiv abgefangen werden. Der kontinuierliche Strom des Liquors durch das Ventrikelsystem und an der Hirnoberfläche entlang erfüllt zusammen mit den Diploevenen außerdem eine Temperaturregelungsfunktion. Gleichzeitig kann auf diesem Weg das Gehirns mit Nährstoffen versorgt und der Abtransport von Stoffwechselendprodukten zur Aufrechterhaltung eines konstanten extrazellulären Milieus realisiert werden (Segal 1993; Lippert 2006). 


\subsection{Pathophysiologische Konzepte}

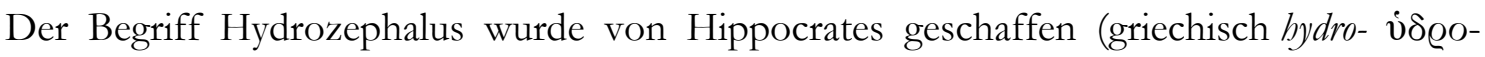

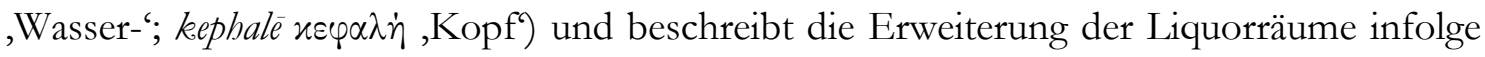
gestörter Liquorzirkulation oder -resorption.

Hier sei bereits angemerkt, dass eine Übersekretion von Liquor als Ursache des Hydrozephalus, zum Beispiel in Folge eines Plexuspapilloms, nur in sehr seltenen Fällen vorkommt und daher im Nachfolgenden nicht weiter ausgeführt wird (Jaiswal et al. 2009).

Der oben bereits erwähnte Vorgang zur Liquorresorption nach Walter Edward Dandy nimmt auch Bezug auf die Kommunikation der verschiedenen Bereiche des Ventrikelsystems zueinander, welche die Grundlage für die Einteilung des Krankheitsbildes bildet und nachfolgend erläutert wird.

Ist der Abfluss von Liquor aus dem inneren System in den Subarachnoidalraum nicht mehr gewährleistet, dann spricht man von einem Verschlusshydrozephalus (Hydrocephalus occlusivus oder Hydrocephalus non-communicans). Funktioniert der Abfluss, aber ist zum Beispiel die Resorption an den pacchionischen Granulationen gestört, nennt man dies einen kommunizierenden Hydrozephalus (Hydrocephalus communicans oder Hydrocephalus malresorptivus) (Gratzl und Merlo 2006).

Davon abzugrenzen ist ein Hydrozephalus infolge einer Hirnatrophie und passiven Liquorraumerweiterung z. B. bei Demenzerkrankungen, welchen man dann Hydrocephalus e vacuo nennt. Außerdem besteht noch die Möglichkeit eines Normaldruckhydrozephalus, dessen pathophysiologische Ursachen wahrscheinlich auf einem erhöhten Druckgradienten zwischen Liquorraum und kortikalen Venen mit darausfolgendem verminderten Liquorabfluss ins venöse System zurückzuführen sind. Die beiden zuletzt genannten Formen sind im Kindesalter sehr selten und daher für die inhaltliche Thematik dieser Arbeit nicht weiter relevant. (Dandy und Blackfan 1914; Rachel 1999). Eine andere Herangehensweise zur Thematik unterteilt die Ursache des HC in angeboren und erworben.

Aufgrund der Vielseitigkeit dieser Möglichkeiten verweisen wir den Leser auf weiterführende Literatur und werden im nachfolgenden Kapitel nochmal explizit auf die Ursachen des infantilen Hydrozephalus eingehen. An dieser Stelle sei angemerkt, dass die schon erwähnten klassischen Einteilungen der vielseitigen Pathologie nur ansatzweise gerecht werden und allenfalls als Unterstützung für den klinischen Alltag angesehen werden können, da diese 
Einteilung des Hydrozephalus maßgeblich für die nachfolgende Therapieentscheidung ist (Jansen et al. 2007).

Der japanische Wissenschaftler Shizuo Oi hat sich Gedanken gemacht, wie es gelingen kann, die verschiedenen Variablen zu bündeln und gleichzeitig Entwicklungen des Krankheitsbildes weiter erfassen zu können. Es entstand auf diesem Weg die „Multicategorical Hydrocephalus Classification”, welche zehn Kategorien mit bis zu 54 Subtypen des HD erfassen kann (Oi 2011).

In dieser Arbeit steht das Krankheitsbild des infantilen Hydrozephalus im Vordergrund. Die Komplexität spiegelt sich nicht nur in der schwierigen pathophysiologischen Einordnung wider, sondern auch in der epidemiologischen Datenlage.

Die Angaben zur jährlichen Inzidenz des kindlichen Hydrozephalus schwanken zwischen 1:1500 und 1:500 (Flannery und Mitchell 2014). Die Prävalenz des angeborenen HC wird mit 0,37-0,81 auf 1000 Geburten angegeben (Stoll et al. 1992; Blackburn und Fineman 1994) und die des postnatal erworbenen mit 0,64-1,6 auf 1000 Geburten (Serlo et al. 1990; Murshid et al. 2000). Einerseits gibt es in der Literatur Hinweise dafür, dass diese Zahlen in den westlichen Regionen mit hohen Einkommen rückläufig sind, da es vorangetrieben durch immer weitere Meilensteine in der pränatalen Medizin, zu einem Rückgang von konnatalen Fehlbildungen durch Schwangerschaftsabbrüche kommt (Persson et al. 2007). Im Gegensatz dazu steht die Tatsache, dass durch eben diese medizinischen Fortschritte die Überlebensrate von Frühgeborenen steigt, welche oftmals einen behandlungswürdigen Hydrozephalus entwickeln (Callaghan et al. 2016). In den Regionen mit geringem Einkommen und hoher Geburtenrate (Lateinamerika und Afrika) werden die höchsten Inzidenzen konnataler Fehlbildungen beobachtet (Dewan et al. 2018).

Gerade bei Kindern bieten die einfachen Pathogenesemodelle eine ausreichende Orientierung in Bezug auf die Ursache für das Hydrozephalusgeschehen. Fast immer ist entweder eine intrazerebrale Läsion (Hydrocephalus occlusivus) oder ein extrazerebrales Durchflusshindernis oder eine Malresorption gerade im Bereich der basalen Zisternen verantwortlich für ein Krankheitsgeschehen (Hydrocephalus communicans) (Rachel 1999).

Nicht nur der Läsionsort, sondern auch der Zeitraum in dem das Krankheitsbild entsteht, ist entscheidend für den klinischen Befund. Akute Hydrozephali, beispielsweise verursacht durch eine Kolloidzyste, sind vor allem durch eine schnelle intrakranielle Druckerhöhungen gekennzeichnet. Eine Aufweitung des Ventrikelsystems steht bei diesem in Stunden bis 
Tagen entstehenden Verlauf nicht unbedingt im Vordergrund. Als subakut oder chronischen Verlauf bezeichnet man einen sich nach mehreren Wochen bis Monaten langsam einstellenden Hydrozephalus. Dabei ist die Ventrikelerweiterung prominent und ausschlaggebend für eine Marklagerschädigung, die dann wiederum zu neurologischen Folgeerkrankungen führen kann. Gleichwohl besteht immer je nach Art und Weise der Störung die Möglichkeit, dass sich ein neues Gleichgewicht zwischen Liquorproduktion und Resorption einstellt, sobald verschiedene Mechanismen der Gegenregulation eingegriffen haben. Man spricht dann von einem arrested bydrocephalus (Muthukumar et al. 2005; Schirmer 2005). An dieser Stelle sei aber auch nochmals erwähnt, dass sämtliche Modellvorstellungen den klinischen Alltag erleichtern können, aber der Realitätsbezug in Frage gestellt werden darf, weil die Komplexität des Gefäßsystems bisher nicht hinreichend in Betracht gezogen wurde (Greitz 2007). 


\subsection{Ursachen}

Für einen orientierenden Überblick kann man die Ursachen ätiologisch grundsätzlich in die gemäß Tabelle 1 dargestellten verschiedenen Gruppen unterteilen.

Tabelle 1: Ursachen des kindlichen Hydrozephalus

\begin{tabular}{|c|c|c|}
\hline & Ursache & Mechanismus \\
\hline \multicolumn{3}{|c|}{ Erworbener Hydrozephalus } \\
\hline \multicolumn{3}{|c|}{ Inflammatorisch } \\
\hline $\begin{array}{l}\text { Subarachnoidale Blutung oder } \\
\text { Infektion }\end{array}$ & Arachnoidale Narbe & Dysfunktionaler Subarachnoidalraum \\
\hline $\begin{array}{l}\text { Intraventrikuläre Blutung oder } \\
\text { Infektion }\end{array}$ & Ependymale Narbe & $\begin{array}{l}\text { Ventrikuläre } \\
\text { Obstruktion/Flusshinderniss }\end{array}$ \\
\hline \multicolumn{3}{|c|}{ Neoplasie } \\
\hline Parenchymaler Hirntumor & Masseeffekt & $\begin{array}{l}\text { Ventrikuläre } \\
\text { Obstruktion/Flusshinderniss }\end{array}$ \\
\hline Spinaler Tumor & Veränderte Liquordynamik & Dysfunktionaler Subarachnoidalraum \\
\hline Metastasierter Tumor & $\begin{array}{l}\text { Tumor mit meningealer } \\
\text { Infiltration }\end{array}$ & Dysfunktionaler Subarachnoidalraum \\
\hline Tumor des Plexus choroideus & Veränderte Liquordynamik & Dysfunktionaler Subarachnoidalraum \\
\hline Tumor des Plexus choroideus & Masseeffekt & $\begin{array}{l}\text { Ventrikuläre } \\
\text { Obstruktion/Flusshinderniss }\end{array}$ \\
\hline $\begin{array}{l}\text { Tumor des Plexus choroideus } \\
\text { oder Hyperplasie }\end{array}$ & Veränderte Plexusfunktion & Liquorüberproduktion \\
\hline \multicolumn{3}{|c|}{ Gefäß-assoziiert } \\
\hline Gefäßmalformation & $\begin{array}{l}\text { Ventrikuläre Obstruktion z.B. } \\
\text { AV-Malformation }\end{array}$ & $\begin{array}{l}\text { Ventrikuläre } \\
\text { Obstruktion/Flusshindernis, } \\
\text { Sinkende Liquorresorption oder } \\
\text { sinkende venöse Compliance }\end{array}$ \\
\hline $\begin{array}{l}\text { Abflussstörung des venösen } \\
\text { Systems }\end{array}$ & $\begin{array}{l}\text { Extrinsische Venenobstruktion } \\
\text { z. B. Sinusvenenthrombose }\end{array}$ & $\begin{array}{l}\text { Sinkende Liquorresorption oder } \\
\text { sinkende venöse Compliance }\end{array}$ \\
\hline \multicolumn{3}{|c|}{ Kongenitaler oder erworbener Hydrozephalus } \\
\hline Angeborene Aquäduktstenose & III. Ventrikel-Obstruktion & $\begin{array}{l}\text { Ventrikuläre } \\
\text { Obstruktion/Flusshindernis }\end{array}$ \\
\hline Neuralrohrdefekte/Dysraphien & $\begin{array}{l}\text { III./IV. Ventrikel-Obstruktion } \\
\text { bzw. arachnoidale oder } \\
\text { ependymale Narbe }\end{array}$ & Variabel \\
\hline $\begin{array}{l}\text { Obstruktion der Fossa cranii } \\
\text { posterior }\end{array}$ & $\begin{array}{l}\text { IV. Ventrikel Obstruktion z.B. } \\
\text { Dandy-Walker- oder Chiari I- } \\
\text { Malformation }\end{array}$ & $\begin{array}{l}\text { Ventrikuläre } \\
\text { Obstruktion/Flusshinderniss }\end{array}$ \\
\hline Zysten & Masseeffekt & $\begin{array}{l}\text { Ventrikuläre } \\
\text { Obstruktion/Flusshinderniss }\end{array}$ \\
\hline $\begin{array}{l}\text { Angeborene Stenose d. Foramen } \\
\text { Monroe }\end{array}$ & $\begin{array}{l}\text { Obstruktion der äußeren } \\
\text { Liquorräume }\end{array}$ & $\begin{array}{l}\text { Ventrikuläre } \\
\text { Obstruktion/Flusshinderniss }\end{array}$ \\
\hline
\end{tabular}

Quelle: (Kahle et al. 2016, S789) 
Angeborene Fehlbildungen, wie zum Beispiel die Aquäduktstenose, eine Chiari-IIFehlbildung bei einer Myelomenigozele, ein Dandy-Walker-Syndrom und diverse Zysten sind sehr häufig mit einem Hydrozephalus assoziiert. Ebenso fatal wirken sich Blutungen auf die Resorption aus. Hierbei sind insbesondere der posthämorrhagische Hydrozephalus infolge einer intraventrikulären Hirnblutung hervorzuheben (Ballabh 2010). Infektionen, wie beispielsweise die bakterielle Meningitis oder Toxoplasmoseenzephalitis können ebenfalls Auswirkungen auf den Liquorfluss haben. Prominent als Ursache eines erworbenen Hydrozephalus im Kindesalter infolge einer Tumorerkrankung ist die Entwicklung eines Medulloblastoms, welches dann bevorzugt in der Medianlinie im IV. Ventrikel entwickelt und dort den Liquorabfluss massiv einschränken kann. Eine andere Tumorerkrankung, welche im Verlauf zum Hydrozephalus führen kann, ist zum Beispiel ein Kraniopharyngeom. Außerdem sind neben Kombinationsmöglichkeiten aus allen oben genannten Formen auch posttraumatische Hydrozephali infolge eines Polytraumas nach einem Verkehrsunfall oder das Bild des kurz angeschnittenen Hypersekretorischen HC infolge eines Plexuspapilloms möglich (Oi 2003; Gratzl und Merlo 2006). 


\subsection{Posthämorrhagischer Hydrozephalus und intraventrikuläre Hirnblutung}

Das insbesondere bei Frühgeborenen vorkommende Krankheitsbild geht auf intrazerebrale Blutungen zurück. Verantwortlich für diese Blutungen sind die Gefäße der noch nicht reifen germinalen Matrix. Dabei spielen viele komplexe Faktoren eine Rolle, die nach wie vor Gegenstand der wissenschaftlichen Untersuchung sind (du Plessis 2009). Momentan wird davon ausgegangen, dass der Pathophysiologie des PHHC eine Fibrose der Arachnoidealzotten und Meningen sowie eine subependymale Gliose zugrunde liegt, welche im Zusammenspiel die Liquorresorption behindern (Cherian et al. 2004). Die genauen molekularen Grundlagen dieses Zusammenhangs sind noch nicht ergründet (Whitelaw und Aquilina 2012). Einen interessanten Ansatz für die Liquorzirkulation beschreiben Greitz et al. (1997), die postulieren, dass die meiste Liquorabsorption entlang des ependymalen Gefäßsystems erfolgt. Die Grundlage zur Diagnose bilden die in Kapitel 2.5 genannten Methoden, insbesondere der Stellenwert der Sonografie soll an dieser Stelle nochmals hervorgehoben werden (Orman et al. 2015). Die Therapie des PHHC umfasst nahezu das gesamte in Kapitel 2.6 erwähnte Repertoire. Mazzola et al. (2014) sind der Auffassung, dass wiederholte Lumbalpunktionen zur Hirndrucksenkung die Anzahl der OP-Indikation für eine permanente Shuntversorgung nicht minimieren können. Dennoch gibt es keinen einheitlichen bzw. wissenschaftlich fundierten Konsens zu dieser Thematik. Der Einsatz von temporären Liquordrainagen, wie subgalealen Reservoiren (RICKHAM) oder externen Ventrikeldrainagen kann eine sinnvolle Option sein, um gerade bei frühgeborenen Kindern unter der 28. SSW Zeit zu gewinnen und das Risiko für Komplikationen durch eine weitere Operation zu minimieren sowie gleichzeitig die Indikation zur Shuntanlage kritisch zu hinterfragen (Köksal und Öktem 2010; Bock et al. 2018a). Der Einsatz von intraventrikulären thrombolytischen Medikamenten (Urokinase, Streptokinase, fibrinspez. Thrombolytika) und die Anwendung von Schleifendiuretika sind nicht geeignet, das Outcome der Patienten zu verbessern. Gleichwohl ist eine stattgehabte intraventrikuläre Blutung nicht zwangsläufig mit der Entwicklung eines PHHCs verbunden. Zu jedem Zeitpunkt und unabhängig vom Blutungsgrad ist eine spontane Senkung des Hirndrucks auf normale Werte möglich. In etwa 10\% bis 20\% der Fälle benötigen die Kinder, unabhängig vom Schweregrad der Blutung und der möglichen Entwicklung eines PHHC, eine permanente Shuntversorgung (Robinson 2012). Die intraventrikulären Blutungen können nach unterschiedlichen Klassifikationen eingeteilt werden. Die bei dieser Dissertation verwendete Klassifikation geht auf die 4 Stufen nach Lou Ann Papile zurück (Papile et al. 
1980). In der beigefügten Tabelle 2 sind die Einteilung nach Papile und die neuere Einteilung der DEGUM dargestellt.

Tabelle 2: Vergleich Klassifikationen intrakranieller Blutungen bei Frühgeborenen (Papile/DEGUM)

\begin{tabular}{|l|l|l|}
\hline \multicolumn{1}{|c|}{ Schweregrad } & \multicolumn{1}{|c|}{ Nach Papile } & \multicolumn{1}{c|}{ Nach DEGUM } \\
\hline Grad I & Subependymale Blutung & Subependymale Blutung \\
\hline Grad II & $\begin{array}{l}\text { Ventrikeleinbruchsblutung } \\
\text { ohne Ventrikeldilatation }\end{array}$ & $\begin{array}{l}\text { Leichte bis mäßige } \\
\text { Ventrikeleinbruchsblutungen }<50 \% \\
\text { d. Ventrikellumens }\end{array}$ \\
\hline Grad III & $\begin{array}{l}\text { Ventrikeleinbruchsblutung } \\
\text { mit Ventrikeldilatation }\end{array}$ & $\begin{array}{l}\text { Schwere } \\
\text { Ventrikeleinbruchsblutung }>50 \% \text { d. } \\
\text { Ventrikellumens }\end{array}$ \\
\hline Grad IV & $\begin{array}{l}\text { Ventrikeleinbruchsblutung } \\
\text { ins Parenchym }\end{array}$ & \\
\hline $\begin{array}{l}\text { Hämorrhagische } \\
\text { Infarzierung } \\
\text { Hirnparenchym }\end{array}$ & & \begin{tabular}{l} 
Seitenangabe, Lokalisation, Größe \\
\hline $\begin{array}{l}\text { Blutung in andere } \\
\text { Hirnareale }\end{array}$
\end{tabular} \\
\hline $\begin{array}{l}\text { Posthämorrhagische } \\
\text { Ventrikelerweiterung }\end{array}$ & & $\begin{array}{l}\text { Basalganglien, Kleinhirn, } \\
\text { Hirnstamm }\end{array}$ \\
\hline
\end{tabular}

Quelle: (Deeg et al. 1999, S. 169) 


\subsection{Klinisches Bild}

Die klinischen Leitsymptome beim kindlichen Hydrozephalus sind sehr variabel und vor allem altersabhängig. Ist der Kopfumfang bei Geburt noch normal, so kann es im Laufe der Zeit zu einem Perzentilensprung des gemessenen Kopfumfangs kommen, welcher als Warnhinweis wahrgenommen werden muss (Poca et al. 2013). Der erhöhte ICP spiegelt sich beim Kind vor allem in einer vorgewölbten Fontanelle und klaffenden Schädelnähten wider (Garton et al. 2001). Aus diesem Grund sind gerade bei den Erstuntersuchungen Kopfumfangsmessungen und Schädelsonografien im Rahmen der Untersuchung von Frühgeborenen entscheidend (Orman et al. 2015). Aus neuropädiatrischer Sicht kann es zu psychomotorischen Entwicklungsstörungen, motorischer Unruhe, Opisthotonus, Spastik und weiteren neurologischen Ausfällen kommen. Sehr bekannt ist auch das sogenannte Sonnenuntergangsphänomen, welches einer vertikalen Blickparese entspricht und bei dem die Kornea unter dem Augenlid verschwindet (Schirmer 2005). Neben den oben genannten Auffälligkeiten lassen sich bei offener Fontanelle Merkmale oder Anzeichen wie ,allgemeine Irritabilität, gestörte Nahrungsaufnahme und kardiopulmonale Instabilität“ (Jansen et al. 2007, S. 88) beobachten. Akutsymptome bei schon geschlossenen Fontanellen wären beispielsweise Nüchternerbrechen, Kopfschmerzen, Übelkeit, Nackenschmerzen und Müdigkeit (Lumenta et al. 2009).

\subsection{Diagnostik}

Im Unterschied zum Erwachsenen gestaltet sich die Diagnostik des kindlichen Hydrozephalus durch die Möglichkeit der Sonografie durch die offene Fontanelle einfacher. Auf diesem Weg können Ventrikelweite und Hirnmanteldicke erfasst werden. Auch eine Verlaufskontrolle in den ersten beiden Jahren ist somit möglich (Brunelle 2005). Voraussetzung dafür ist jedoch, dass moderne Geräte mit der Möglichkeit zum 2D oder 3D Ultraschall verwendet werden (Orman et al. 2015). Bei unklarer Herkunft des HC ist ein MRT Mittel der Wahl zur Ursachenidentifizierung, wobei jedoch beachtet werden muss, dass auf diesem Weg keine Aussagen über den Liquordruck gemacht werden können. Dieser kann direkt über eine Hirndruckmessung erfasst werden oder aber indirekt über eine Druckmessung bei noch offener Fontanelle (Fontanometrie) (Schirmer 2005). Anzeichen für einen Hydrozephalus können in T1 und T2 gewichteten MRT-Aufnahmen erhöhte Signalintensitäten sein, welche sich vor allem periventrikulär manifestieren (Schirmer 2005). 
Ebenso möglich wäre ein CT, welches jedoch eine erhöhte Strahlenbelastung für das noch nicht fertig entwickelte Gehirn und die Augenlinse mit sich trägt. Eine mögliche Folgeschädigung der Linse mit Katarakt muss unbedingt abgewogen werden. Das früher standardmäßige Nativröntgen ist heutzutage nicht mehr Goldstandard und wird allenfalls bei einer Abdomenübersichtsaufnahme bei Kindern im Wachstumsalter zur Beurteilung der Länge des verbleibenden Shunts im Bauchraum verwendet (Jansen et al. 2007). Dennoch kann bei fehlender klinischer Symptomatik eine Beurteilung der Schädelnähte hinweisend sein. Das Bild eines „Wolkenschädels“ ist heutzutage allerdings nicht mehr typisch (Schirmer 2005; Lumenta et al. 2009).

\subsection{Therapieoptionen des kindlichen Hydrozephalus}

Das nachfolgende Kapitel soll einen kurzen Überblick über mögliche Therapieoptionen des kindlichen Hydrozephalus geben und dabei den derzeitigen Stand der Forschung erörtern. An dieser Stelle muss erwähnt werden, dass eine kausale kurative Therapie des HC nur in ausgewählten Einzelfällen möglich ist und die gängige Behandlungsmethode der Anlage eines Shuntsystems palliativer Natur ist.

\subsubsection{Pharmakologische Therapieoptionen}

Die pharmakologischen Optionen zur Hirndrucksenkung sind nach jetzigem Stand sehr begrenzt und wenig vielversprechend. Es gibt die Möglichkeit, durch Carboanhydrasehemmer wie Acetazolamid in Kombination mit dem Schleifendiuretikum Furosemid die Liquorproduktion zu vermindern (Elvidge et al. 1957). Unerwünschte Nebenwirkungen sind jedoch teilweise extreme Störungen des Säure-Basen-Haushalts (Ketoazidose) und gerade bei Frühgeborenen die Entstehung einer Nephrokalzinose (Narendra et al. 2001). Dennoch ist die Bedeutung der Medikation nicht zu vernachlässigen, denn gerade eine temporäre kurzfristige konservative Behandlung kann eine weitere Entwicklung des Kindes zulassen und schafft damit die Voraussetzung zur operativen, definitiven Shuntversorgung (Stephani et al. 1989). Dem entgegen stehen Langzeitstudien, welche gerade beim häufigen Krankheitsbild des PHHC von einer erhöhten Shuntanlagenrate und erhöhter neurologischer Morbidität unter medikamentöser Therapie ausgehen (International PHVD Drug Trial Group 1998). Rückblickend auf die letzten 50 Jahre wurde noch keine medikamentöse Option zur zufriedenstellenden Behandlung des Hydrozephalus gefunden (Del Bigio und Di Curzio 2016). 


\subsubsection{Neuroendoskopie: ETV (Endoscopic Third Ventriculostomy)}

Den Grundstein für die kurative Behandlung des Hydrozephalus legte Walter E. Dandy schon 1922 mit seiner Technik der Ventrikulostomie. Dabei durchstieß er die Lamina terminalis des dritten Ventrikels und schuf auf diesem Weg eine direkte Verbindung zwischen innerem und äußerem Liquorraum. Nach den ersten Jahren des Enthusiasmus musste er jedoch feststellen, dass zum damaligen Zeitpunkt der Erfolg des Verfahrens begrenzt war (Decq et al. 2013). Erst die Weiterentwicklung des Verfahrens und die verbesserten Materialien am Ende des 20. Jahrhunderts führten zu einem Wiedererwachen des Interesses an der Ventrikulostomie als Alternative zum Shuntsystem (Jones et al. 1990). Das Verfahren funktioniert nun wie folgt: Um den anatomisch vorgesehenen Weg des Liquors vom dritten Ventrikel über den Aquädukt in den vierten Ventrikel zu überbrücken, der zum Beispiel infolge einer angeborenen Aquäduktstenose verlegt ist, schafft man sich endoskopisch einen künstlichen Zugang im Boden des dritten Ventrikels zur Cisterna interpeduncularis. Auf diesem Weg besteht ein kontinuierlicher Fluss zwischen allen Liquorreservoiren (Morello und Migliavacca 1959). Ein eindeutiger Vorteil dieser Methode ist die Vermeidung der Implantation von Fremdkörpermaterial gepaart mit einer minimalinvasiven Zugangsweise (Cinalli et al. 2005). Auftretende Komplikationen sind beispielsweise intraoperative Blutungen, dabei ist insbesondere eine $\operatorname{Ruptur} \operatorname{der} A$. basilaris gefährlich oder postoperative Infektionen. Die Erfolgsrate der ETV ist stark abhängig vom Alter des Patienten. Insbesondere die Anwendung bei Kindern unter 12 Monaten ist mit einer höheren Komplikationsrate und geringeren Erfolgsrate verbunden (Bouras und Sgouros 2011). Um das Outcome noch weiter zu verbessern, gibt es die Möglichkeit, den sogenannten ETV Success Score anzuwenden (Furlanetti et al. 2012). Auch eine Kombination des Verfahrens mit Neuronavigation ist möglich und bringt einen Vorteil für den Operationsvorgang (Rohde et al. 2011). Eine weitere Modernisierung des Verfahrens ist der Einsatz von laserassistierter Neuroendoskopie. Dabei wird ein $2 \mu \mathrm{m}$ Nah-Infrarot Laser für den operativen Zugang von Zysten und drittem Ventrikel verwendet. Mit seiner ablativen und koagulierenden Wirkung ist dieser sehr gut für schwierige Operationsbedingungen geeignet (Ludwig et al. 2007; Schuhmann et al. 2019).

Abschließend sei gesagt, dass die neuesten Erkenntnisse bezüglich der Erfolgsrate zwischen Shunt und EVT anscheinend annährend gleich sind (Limbrick et al. 2014; Kulkarni et al. 2016), aber gerade der Hydrozephalus occlusivus primär mit einer endoskopischen Fensterung behandelt werden sollte (De Ribaupierre et al. 2007; Khan et al. 2016). Weitere Studien bemühen sich, dies weiter zu untersuchen, um somit die Grundlage für einheitliche Leitlinien zu legen (Kulkarni et al. 2016). Zusätzlich gibt es Hinweise, dass die ETV eine 
Alternative zur Shuntrevision nach zum Beispiel durchgemachter Infektion darstellt und die Kinder auch in Bezug auf weitere mögliche Komplikationen von dieser Behandlungsoption profitieren können (Bilginer et al. 2009; Marton et al. 2010). Entgegen aller Begeisterung für das neue Verfahren, muss die Art und Weise der Behandlung eines kindlichen Hydrozephalus stets im engen Dialog mit den Eltern des Kindes erfolgen. Weiterhin ist diese Behandlungsoption ein invasiver Eingriff, dessen Erfolg bei aller Sicherheit und Planung anwenderspezifische Risiken mit sich bringt.

\subsubsection{Aquäduktoplastie}

Aquäduktoplastie bezeichnet die endoskopische Wiederherstellung des Liquorflusses im Aquädukt. Dieser kann beispielsweise aufgrund eines Verschlusshydrozephalus kompromittiert sein. Es erfolgt dann eine endoskopische Erweiterung der Äquäduktstenose. Dies kann durch Perforation einer vorhandenen Membran oder Stenteinlage mithilfe von Siliconkatheter durchgeführt werden. Die Technik hat als Ziel, den natürlichen Liquorabfluss zu erhalten. Je nach Zugangsweg erfolgt die Aufdehnung dann mit einem Ballonkatheter (Schroeder und Gaab 1999) und mit oder ohne anschließende Stenteinlage. Es können dabei allerdings die um den Aquädukt liegenden Strukturen des Mittelhirns verletzt werden, sodass es infolgedessen beispielsweise zu Augenbewegungsstörungen kommen kann (Geng et al. 2015).

Fritsch und Schroeder (2013) schlagen vor, dass die Aquäduktoplastie mit Stenteinlage vor allem bei der Behandlung von einem isolierten vierten Ventrikel in Betracht gezogen werden sollte. Eine Aquäduktoplastie alleine hat eine hohe Verschlussrate und sollte eher vermieden werden. Bei Verlegungen des Aquädukts durch Tumorerkrankungen sollte eher eine ETV bevorzugt werden (Sagan et al. 2006). 


\subsubsection{Plexus-Choroideus-Kauterisation}

Eine weitere Behandlungsmethode ist die Gewebekauterisation, bei der die Liquorproduktion des Plexus Choroideus durch eine interventionelle elektrokauterisierende Maßnahme gedrosselt werden soll (CPC). Der alleinige therapeutische Nutzen für die Behandlung des kindlichen Hydrozephalus ist gering. Es gibt aber die Möglichkeit, eine CPC mit der EVT zu kombinieren und damit das Outcome der Kinder zu verbessern (Warf 2013; Chamiraju et al. 2014; Shitsama et al. 2014). Gegen eine CPC spricht die einzigartige Funktion des Plexus und seiner Ausstattung mit Folatrezeptoren zur Verteilung in das ZNS (Wollack et al. 2007).

\subsubsection{Shuntoperationen}

Nach diversen gescheiterten bzw. nicht ausgereiften Behandlungsversuchen begründeten Frank Nulsen und Eugen Spitz im Jahre 1949 nach Verfügbarkeit der modernen Siliconmaterialien die moderne Hydrozephalustherapie mit der erfolgreichen Implantation eines Shuntsystems mit einem Kugelventil in die Vena jugularis (Nulsen und Spitz 1951). Den nächsten Meilenstein erreichte John D. Holter im Kampf um das Leben seines Sohnes, der an einem infantilen Hydrozephalus erkrankte. Er entwickelte als erster das DoppelSilikon-Schlitz-Ventil mit einer Helix-Feder, welches die Grundlage für die Massenproduktion von Shuntsystemen war und 1956 erstmals von Eugen Spitz implantiert wurde (Aschoff et al. 1999). Heutzutage gibt es viele verschiedene Ventilsysteme mit ebenso vielen zugehörigen unterschiedlichen Shuntsystemen und die Entwicklungen sind noch lange nicht abgeschlossen (Drake et al. 1998). Immer wieder werden neue Innovationen gefunden, wobei darauf hingewiesen werden muss, dass jede neue Errungenschaft auch mit neuen Komplikationen einhergehen kann (Drake et al. 2000).

Die chirurgisch palliativen Maßnahmen der Shuntversorgung teilen sich auf in interne und externe Ableitungen. Für vorübergehende Druckentlastung kann man beim infantilen Hydrozephalus die Vorderhörner des Ventrikelsystems punktieren und in ein offenes oder geschlossenes externes Drainagesystem ableiten. Dabei hat sich gerade bei Frühgeborenen mit intraventrikulären Blutungen die Anlage eines subgalealen Reservoirs (RICKHAM Kapsel) für vorübergehende intermittierende Punktionen bewährt. Es besteht aus einer halbrunden Silikonkapsel, welche kurz unter der Haut liegt und mit einem Ventrikelkatheter Zugang zum Liquorsystem hat (Rickham 1964; Mazzola et al. 2014).

Die internen Versorgungsmöglichkeiten des kindlichen Hydrozephalus stehen ganz im Zeichen der Shuntsysteme. Die Möglichkeiten von Liquorshuntsystemen sind sehr vielfältig 
und bevorzugte Therapiemethode, falls die Ursache des HD nicht beseitigt werden kann.

Tabelle 3: Distale historische Ableitungsoptionen zur Liquorresorption

\begin{tabular}{|l|l|l|}
\hline \multicolumn{1}{|c|}{ Kopf- und Halsbereich } & \multicolumn{1}{c|}{ Abdominale Region } & \multicolumn{1}{c|}{ Brusthöhle } \\
\hline Subgaleal & Bauchhöhle & Ductus thoracicus \\
\hline Sinus sagittalis sup. & Bursa Omentalis & Epiduralraum der WS \\
\hline V. jugularis int. & Magen & Knochenmark \\
\hline V. facialis & Gallenblase & Pleuraspalt \\
\hline Subduralraum & Ileum & Rechter Vorhof \\
\hline Subarachnoidalraum & Ureter & V. Cava sup. \\
\hline Mastoid Antrum & Eileiter & \\
\hline Speichelgang & & \\
\hline Qung & & \\
\hline
\end{tabular}

Quelle: McCullough (1986)

Tabelle 3 gibt eine Übersicht über mögliche Ableitungsoptionen. Ebenso greifen Morosanu et al. (2018) das Thema auf und geben einen Überblick. Die heutzutage am häufigsten verwendeten Verfahren sind die ventrikulo-peritoneale oder ventrikulo-atriale Ableitung. Dabei handelt es sich um subkutane Schlauchsysteme, die den Liquor aus den Seitenventrikeln entweder in die Bauchhöhle oder den rechten Vorhof des Herzens ableiten. Dort kann dieser dann über das Peritoneum oder über den regulären Blutkreislauf wieder dem Gesamtsystem zugeführt werden. Es wird somit ein künstlicher Abfluss in ein anderes Körperkompartiment geschaffen (Schirmer 2005). In das Schlauchsystem ist ein regelbares Ventilsystem integriert, welches im Endeffekt im Kopf für einen physiologischen Liquordruck sorgen soll. Zusätzlich wird der Rückstrom durch ein Rückschlagventil verhindert, welches damit gleichzeitig die wichtige Funktion der Infektionsbarriere beinhaltet. Die drainierte Menge wird durch den notwendigen Öffnungsdruck des Ventils bestimmt. Dieser darf jedoch weder zu groß (Überdrainage), noch zu klein (Unterdrainage) sein. Die dabei wirkenden physikalischen Druckunterschiede zwischen hydrostatischem, intrakraniellem und intraperitonealem Druck sind hochkomplex und von verschiedenen Faktoren, wie zum Beispiel der Lage des Patienten abhängig. Hinzu kommt beim infantilen HC das schnelle Wachstum der Patienten, welches die Vorgänge weiter verkompliziert (Aschoff et al. 1999).

Für die Thematik dieser Arbeit steht insbesondere die ventrikulo-peritoneale Ableitung im Vordergrund, welche aus diesem Grund in ihrer Gesamtheit näher erläutert wird. Die Entscheidung für die Versorgung eines Kindes mit einem Shunt ist fast immer eine 
Lebensentscheidung, denn meist bleiben die Shunts lebenslang implantiert. Aus diesem Grund sind die genaue Indikationsstellung und der richtige Implantationszeitpunkt von enormer Wichtigkeit (Tuli et al. 2000). Obwohl es Tendenzen gibt, die erkennen lassen, dass eine möglichst späte Shuntanlage nicht nur das Outcome für den ersten Shunt verbessert, sondern auch die Revisionsrate niedriger sein soll (Tuli et al. 2000; Riva-Cambrin et al. 2015), ist der Implantationszeitpunkt in der aktuellen Forschung nach wie vor umstritten. Gleichzeitig soll das Risiko für neurologische Folgeerkrankungen trotz der verzögerten definitiven Versorgung nicht erhöht sein (Taylor und Peter 2001).

Die Misserfolgsrate für alle implantierten Shuntsysteme ist hoch und liegt bei ca. $40 \%$ im ersten Jahr und ca. 50\% im Verlauf von zwei Jahren. Die Art, das Design oder die Ventilart sind hierbei nicht entscheidend (Baird et al. 2014). Die Erfolgsrate ist bei allen Ableitungsmöglichkeiten wenig zufriedenstellend (Pollack et al. 1999; Kestle et al. 2000; Browd et al. 2006a; Beez et al. 2014). Die Ursachen für diese negativen Ergebnisse der Statistiken begründen sich vor allem durch die große Bandbreite der möglichen Komplikationsursachen wie beispielsweise Infektionen, Obstruktionen oder mechanisches Versagen, welche im weiteren Kontext erklärt werden. Dabei wird insbesondere auf die abdominellen Komplikationen nach ventrikulo-peritonealer Shuntanlage näher eingegangen. 


\subsection{Shuntkomplikationen und Shuntversagen}

Die Symptome für ein Shuntversagen sind ebenso vielfältig und komplex wie die Manifestationssymptome des kindlichen Hydrozephalus und reichen von Übelkeit, Erbrechen, Verwirrtheit oder Fieber bis zu allgemeiner Schwäche und Visusverlust. Gerade bei Kindern stehen dabei eine gespannte Fontanelle und aufgeweitete Suturen mit Kopfumfangszunahmen im Vordergrund. In der neurologischen Untersuchung lassen sich häufig Hyperreflexien, Papillenödeme oder Augenbewegungsstörungen nachweisen. Gleichzeitig kann bei einer Untersuchung des Shunts in seinem Verlauf unter der Haut eine mögliche mechanische Diskonnektion getastet oder im Falle einer Infektion ein Erythem beobachtet werden. Nützlich ist auch eine Palpation des Abdomens, welche Hinweise auf eine Raumforderung, wie beispielsweise bei einer Liquorzyste geben kann (Browd et al. 2006a). Laut Garton et al. (2001) liegt ein positiver Prädikationswert insbesondere bei Übelkeit und Erbrechen (79\%), erhöhter Berührungsempfindlichkeit (78\%), Bewusstseinsbeeinträchtigungen (100\%) und gespannter Fontanelle ( $92 \%)$ vor. Hinweise lassen sich außerdem auch durch Befragung der Eltern finden, die bei ihrem Kind die bereits aufgetretene Shuntkomplikation wiedererkennen und benennen können (Neiter et al. 2016). Dem klinischen Verdacht einer Shuntfehlfunktion wird infolge weiterer Untersuchungen nachgegangen. Dabei sind die im Kapitel 2.6. aufgeführten Methoden hilfreich. Eine weitere diagnostische Option zusätzlich zu den schon genannten Möglichkeiten ist der Einsatz von Shuntogrammen. Dabei wird das bereits bestehende Shuntsystem mit Kontrastmittel gefüllt und eine röntgenologische Kontrolle durchgeführt. Insbesondere Shuntokklusionen sind gut zu validieren bei geringer Strahlenbelastung (Eckardstein et al. 2017). In diesem Zusammenhang sei zu erwähnen, dass insbesondere ein multimodaler diagnostischer Ansatz und die Erfahrung des behandelnden Arztes für eine schnelle Versorgung des Kindes ausschlaggebend sind (Browd et al. 2006a). 


\subsubsection{Infektion des Shuntsystems}

Die Infektion ist eine häufige Komplikation bei der Implantation eines ventrikuloperitonealen Shuntsystems. Die Häufigkeit wird in der Literatur allerdings mit unterschiedlichen Wahrscheinlichkeiten von 3\% bis 27,6\% angegeben.

Tabelle 4: Studienübersicht zur Infektionsrate und Inzidenz bei VP-Shunts

\begin{tabular}{|c|c|c|c|c|c|c|}
\hline & & & & \multicolumn{2}{|c|}{ Infektionsrate } & \\
\hline Autor & Jahr & $\begin{array}{c}\text { Patienten/ } \\
\text { Operationen }\end{array}$ & $\begin{array}{c}\text { Zeitraum } \\
\text { (Jahre) }\end{array}$ & Fälle & Prozent (\%) & $\begin{array}{c}\text { Inzidenz } \\
\mathbf{( \% )}\end{array}$ \\
\hline Odio et al. & 1984 & $297 / 516$ & 7 & 59 & 11 & 19 \\
\hline Dallacasa et al. & 1995 & $81 / 191$ & 12 & 14 & 7,8 & 17,2 \\
\hline Ronan et al. & 1995 & $? / 900$ & 10 & 41 & 4,5 & k.A. \\
\hline Rotim et al. & 1997 & $201 / 382$ & 4,5 & 36 & 9,4 & 17,9 \\
\hline Mancao et al. & 1998 & $145 / 268$ & 6 & 29 & 10,8 & 20 \\
\hline Baird et al. & 1999 & $? / 957$ & 10 & 94 & 8,7 & k.A. \\
\hline Davis et al. & 1999 & $1193 / 2325$ & 10 & 74 & 3 & 6 \\
\hline Bruinsma et al. & 2000 & $70 / ?$ & 7 & 21 & k.A. & 30 \\
\hline Lan et al. & 2003 & $129 / ?$ & 4,5 & 10 & k.A. & 7,7 \\
\hline Braga et al. & 2009 & $46 / 98$ & 2 & 8 & 19,5 & k.A. \\
\hline Lima et al. & 2007 & $587 / ?$ & k.A. & 16 & 27,6 & k.A. \\
\hline
\end{tabular}

k.A. $=$ Keine Angaben

Quelle: Prusseit et al. (2009) S. 326

Risikofaktoren für eine Shuntinfektion sind unter anderem: Frühgeburtlichkeit, intraventrikuläre Hirnblutung, Wundheilungsstörungen und mangelnde Erfahrung seitens des Operateurs (Prusseit et al. 2009).

Die meisten Infektionen sind durch grampositive opportunistische transiente Hautkeime, insbesondere durch den Biofilm-affinen Staphylococcus epidermidis und Staphylococcus aureus verursacht. Darüber hinaus werden auch Koagulase-negative Keime als Ursache angegeben (Prusseit et al. 2009; Steinbok et al. 2010).

Zeitlich manifestieren sich die Entzündungsreaktionen zu 80\% innerhalb der ersten drei Monate nach Implantation und 90\% innerhalb der ersten sechs Monate, was darauf schließen lässt, dass gerade intraoperativ oder in der angrenzenden postoperativen Phase der Wundheilung eine Kontamination der Wunde erfolgte (Key et al. 1995). Spätinfektionen sind 
seltener und lassen sich in vielen Fällen auf intraabdominelle Liquorzysten oder auf bakterielle Sekundärinfektionen zurückführen (Baird et al. 1999) .

Die Anzeichen einer Shuntinfektion sind oftmals eher unspezifisch oder verlaufen inapparent. Das Auftreten von Fieber ist hierbei eine der häufigsten Begleiterscheinungen. Diagnostisch hinweisend ist eine Liquorpleiocytose $(>15 / \mu \mathrm{l}$; bei Neonaten: $>32 / \mu \mathrm{l}) \mathrm{mit}$ verbundener klinischer Symptomatik. In einigen Fällen ist die Diagnosestellung sehr schwierig und langjährige klinische Erfahrung gefordert.

Ebenso komplex ist das therapeutische Management der Shuntinfektion. Viele Entscheidungen beruhen dort auf Erfahrungswerten, die wissenschaftliche Datenlage ist uneinheitlich. Tamber et al. (2014) versuchten in diesem Zusammenhang, die verschiedenen Therapieoptionen zu überblicken und eine Entscheidungshilfe zu liefern.

Deren abschließende Empfehlungen beinhalten mit den Evidenzleveln II und III eine eher mäßige Grundlage. Sie schlussfolgern, dass die Basis der Behandlung ein multimodaler Ansatz sein muss, dessen Anker die systemische Antibiose ist. Weiterhin ist es möglich, das Shuntsystem partiell zu externalisieren oder aber einen kompletten Austausch durchzuführen, um die Infektionsquelle zu entfernen. Die intrathekale Anwendung von Antibiotika ist nach wie vor umstritten. In diesem Zusammenhang wird deutlich, dass keine einheitlichen Empfehlungen für die optimale Durchführung der systemischen Antibiose oder ein standardisiertes Behandlungsprotokoll zur Verfügungen stehen. Die Indikation zu weiteren prospektiven Studien ist hier eindeutig gegeben. Einen positiven Nutzen für das Patientenoutcome könnte auch der Einsatz von Antibiotika-imprägnierten Shuntsystemen sein. Hierbei finden insbesondere die Wirkstoffe Rifampicin und Clindamycin Verwendung (Aryan et al. 2005; Klimo et al. 2014, S. 7).

Abschließend zum Thema Shuntinfektionen sei jedoch gesagt, dass die effektivste Methode zur Vermeidung von Komplikationen die genaue Untersuchung der Notwendigkeit dieses therapeutischen Schrittes ist.

„The safest method for preventing shunt infection is to not implant a shunt system at all (Prusseit et al. 2009, S. 330)." 


\subsubsection{Mechanische und physikalische Probleme}

Neben der infektiösen Komponente ist die Palette anderer Probleme infolge einer Shuntinsertion weit gefächert. Shuntobstruktionen, mechanisches Versagen, Überdrainage, die Entwicklung von Kompartimenten im Ventrikelsystem und abdominelle Probleme spielen eine wichtige Rolle. Typischerweise hat fast jede Komplikation ein zum Therapieverlauf passendes Zeitfenster, in dem sie gehäuft auftritt.

Eine Ausnahme ist die Shuntobstruktion, welche sich zu jedem Zeitpunkt entwickeln kann und die häufigste Komplikation des Shuntversagens darstellt (Vernet et al. 1995). Die wichtigsten Läsionsorte sind Ventrikelkatheterspitze und Ventil. Im Kopf kann ein Einwachsen des Plexus Choroideus in die Perforationen des Ventrikelkatheters den Shuntabfluss kompromittieren. Das Ventil ist häufig durch Blut oder Zellreste in seiner Funktion beeinträchtigt (Collins et al. 1978). Seltener ist eine Verstopfung des distalen Katheters durch Adhäsionen im Bauchraum, Vernarbungen oder Verdrillungen des Katheterendes. Therapeutische Konsequenz nach Diagnose ist die chirurgische Revision, welche auf verschiedene Art und Weise erfolgen kann und vor allem vom Läsionsort abhängig ist (Browd et al. 2006a).

Ein mechanisches Versagen des Shunts ist selten und in den meisten Fällen einem Materialfehler oder einem Operationsfehler geschuldet. Dabei kann man ferner zwischen Shuntbruch und Diskonnektion unterscheiden. Die klinische Symptomatik ist dabei eher gering ausgeprägt und äußert sich beispielsweise durch einen leichten Anstieg des ICPs, verbunden mit leichten Schwellungen und Erythemen entlang des Shuntverlaufs. Ein Bruch des Shunts manifestiert sich oft erst nach Jahren des problemlosen Verlaufs. Gerade bei Kindern ist dies eine sehr seltene Variation des Shuntversagens, denn oftmals geht ein wachstumsbedingter Wechsel des Systems einer Materialermüdung voraus (Browd et al. 2006a). Infolge immunologischer Prozesse kann es jedoch zu einem Einwachsen des Shunts und dem Entstehen von Narbengewebe um das Silikon herum kommen (Del Bigio 1998). Die Bewegungsfreiheit des Systems wird dadurch eingeschränkt und entstehende Scherkräfte, begünstigt durch das Wachstum und chemische Veränderungen an den Siliconpolymeren, können zu Materialbrüchen führen (Morishita et al. 2002). Der Hauptlokalisationsort dieser Bruchfugen ist dann vornehmlich der Bereich um die Klavikula oder die Region der unteren Rippen. Es ist nicht ungewöhnlich, dass teilweise sehr große Lücken entdeckt werden oder eine Hälfte des Shuntschlauches komplett in die peritoneale Höhle disloziert ist. Dieses Szenario kann auch infolge einer Diskonnektion der beiden 
Bestandteile Ventrikelkatheter und Peritonealkatheter entstehen. Mögliche Ursachen sind hierbei gerissene oder nicht fest genug angelegte chirurgische Knoten. Zeitlich ordnen sich diese Komplikationen aber im Gegensatz zum Shuntbruch eher früher nach dem Operationsdatum ein. Auffallend sind dann oftmals Flüssigkeitsansammlungen an der betroffenen Stelle, die sich mitunter gut palpieren lassen (Browd et al. 2006a). Es gibt aber auch Situationen, in denen nur ein kleiner Teil des Shuntsystems verloren geht und der restliche Stumpf den Liquorfluss aufrechterhalten kann. Diese Fälle können teilweise über Jahre asymptomatisch bleiben (Vinchon und Dhellemmes 2005). Mit radiologischen Shuntserien lassen sich Materialbrüche und Shuntdiskonnektionen sehr sensitiv nachweisen. Ein CT oder MRT kann ebenso weiteren Aufschluss geben (Sivaganesan et al. 2012). Ein Versuch der Einführung eines durchgängigen Shuntsystems ohne Verbindungskomponente konnte sich bisher, auch aufgrund der vom Standard abweichenden operativen Anlagetechnik nicht durchsetzen. Auch die Beschichtung der Silikonoberflächen mit Gel zur Vermeidung von Gewebsbrückenbildungen ist noch nicht ausgereift (Del Bigio 1998; Browd et al. 2006a; Sarkiss et al. 2014). Im Falle eines in die Bauchhöhle disloziertem VPS, mit gleichzeitig insuffizienter Liquordrainage, ist eine operative Bergung (offen oder laparoskopisch) indiziert. (Vinchon und Dhellemmes 2005).

\subsubsection{Migration des Shunts}

Die Migration eines Shunts in ein anderes Gewebe kann ursächlich für eine Fehlfunktion sein. Dabei kann es entweder intraventrikulär zu einer nicht gewünschten Lageveränderung der Katheterspitze gekommen sein oder das distale Ende in der Bauchhöhle liegt an einer Stelle, an der keine wirksame Drainage des Liquors mehr erfolgen kann. Ähnlich wie bei den anderen, nicht infektiösen Shuntkomplikationen können Wachstumsprozesse oder körperkonstitutionelle Veränderungen die ursprüngliche suffiziente Lage des Shuntsystems kompromittieren. Beispielsweise ist es möglich, dass der intraperitoneale Schenkel in das Kolon, Rektum, die Harnblase oder Vagina eindringt. Die Eindringwahrscheinlichkeit ist dabei von der Katheterart abhängig. Schwachstellen im Peritoneum zusammen mit positiven intrabdominellen Drücken oder aber negative Drücke im Thorax können eine Migration zur Folge haben (Popa et al. 2009). Rein klinisch lässt sich diese Diagnose sehr selten eindeutig stellen, da die Symptome sehr unspezifisch sind. Hierfür ist eine weitere radiologische Abklärung mit CT/MRT oder radiologischer Leeraufnahme erforderlich (Browd et al. 2006a). Neben der künstlichen nachträglichen Migration in andere Gewebe ist auch eine falsche Anlage des VPS eine mögliche Komplikation. Fälschlich eingeschätzte Shuntlängen 
oder ein fehlerhafter anatomischer Zugang können einer erfolgreichen Ableitung im Wege stehen und eine abdominale Komplikation nach sich ziehen.

\subsection{4 Überdrainage und Slit-Ventrikel-Syndrom}

Allen bisher genannten Shuntkomplikationen liegt als Hauptproblem ein verminderter oder nicht suffizienter Liquorabfluss zugrunde. Es gibt aber auch die Möglichkeit, dass der Shunt überdrainiert und dem Ventrikelsystem zu viel Liquor entzogen wird. Das dabei entstehende symptomatische Krankheitsbild ähnelt dem einer Shuntfehlfunktion und ist selten klar davon abzugrenzen. Es können Kopfschmerzen, Übelkeit und Erbrechen auftreten, in seltenen Fällen auch Lethargie und Koma. Ein wichtiger Unterschied im Gegensatz zu den vorher genannten Symptomen ist, dass diese häufig nach einem Lagewechsel auftreten und sich teilweise in Rückenlage wieder verbessern. Die Problematik der Überdrainage kann entweder sehr früh nach der Shuntanlage auftreten und ist dann oft mit extraaxialen Flüssigkeitsansammlungen beziehungsweise subduralem Hämatom verbunden; oder sie tritt erstmals lange Zeit nach der Shuntanlage auf und stellt sich dann mit sehr schmalen, engen Ventrikeln dar (Sog. slit-ventricle-syndrome kurz: SVS) (Browd et al. 2006b). Laut Major et al. (1994) liegt für diese Langzeitkomplikation zwischen Shuntinsertion und auftretender Symptomatik eine Zeitspanne von durchschnittlich 6,5 Jahren. Die Inzidenz dafür ist niedrig und variiert je nach Studienlage zwischen 1,8\% (Vernet et al. 1995) und 10\% (Sgouros et al. 1995) oder nach Rekate (2005) bis zu 15\%. Als Therapieoptionen kommen konservative oder operative Optionen in Betracht. Gerade bei dem akuten Auftreten ohne fehlende Hirnkompression oder der Gefahr einer Herniation des Hirnstamms kann eine behutsame Umstellung des Ventils auf einen höheren Öffnungsdruck für die Behandlung ausreichend sein. Auch Patienten mit einem SVS deren Symptome unregelmäßig auftreten und den Alltag gar nicht oder nur geringfügig beeinflussen, sollten eher abwartend und überwachend und gegebenenfalls mit ergänzender medikamentöser Therapie behandelt werden. Schlägt der konservative Ansatz fehl, ist die operative Variante indiziert. Hierbei kommt die Shuntrevision mit Ventiltausch oder die äußere Trepanation der angestauten Flüssigkeit infrage. Ungeachtet der Behandlungsoptionen ist die Patientenklientel anfällig für weitere Shuntkomplikationen (Rekate 2005; Browd et al. 2006b). 


\subsubsection{Nachträgliche Kompartimentierung des Liquorraums}

Bei Kindern mit stattgehabter Ventrikulitis oder intraventrikulärer Blutung besteht das Risiko, dass es innerhalb des Ventrikelsystems zu einer Kompartimentierung kommt. Dabei entsteht in gewisser Weise ein interner Hydrozephalus non-communicans und ein einzelner Shunt schafft es nicht, das gesamte System erfolgreich zu drainieren. Meistens zeigen sich diese Taschenbildungen nicht initial, sondern werden erst im Laufe der Zeit bemerkt, wenn die Ventrikel sich im separierten Teil weiter ausdehnen und es zu einem Anstieg des ICP's kommt. Für die Diagnostik dieser Kompartimente kommt in erster Linie das MRT in Frage. Als Therapieoption ist die endoskopische Fensterung der Kompartimente und Wiederherstellung des Liquorflusses durch die Kommunikation anzustreben. Ist dies nicht realisierbar, ist eine Erweiterung des Shuntsystems und Anlage eines zweiten Ventrikelkatheters erforderlich. Eine Therapie bei asymptomatischen Kindern ist nicht zwingend notwendig (Spennato et al. 2005; Browd et al. 2006b). 


\subsection{Abdominelle Komplikationen peritonealer Shunts}

Neben den bereits genannten Komplikationen ventrikulo-peritonealer Shunts liegt in dieser Arbeit der Schwerpunkt auf den speziellen und weniger häufig auftretenden abdominellen Komplikationen, welche aus diesem Grund näher beleuchtet werden sollen.

Mit der Einführung des peritonealen Shuntsystems haben sich die Prognosen für Kinder mit einem Hydrozephalus deutlich verbessert. Gleichwohl tat sich damit auch ein neues Feld von möglichen Komplikationen auf. Das Einbringen eines Fremdkörpers in eine Körperhöhle und damit die Beeinflussung des physiologischen Gleichgewichts geht nicht ohne Pathologien von statten. Hinzu kommt beispielsweise die Appendizitis oder eine mögliche Schwangerschaft, welche in Kombination mit einem bestehenden Shunt zu Problemen führen können. Andere Möglichkeiten abdomineller Komplikationen sind neu auftretende oder angeborene Hernien, Hydrozelen, Liquorpseudozysten, in den Bauchraum dislozierte Shuntkomponenten nach einem Materialbruch oder Organwandperforationen. Auch die Möglichkeit der Entstehung von Aszites durch mangelnde Liquorresorption des Peritoneums oder Begleiterkrankungen wie Herzinsuffizienz, Leberzirrhosen oder ähnliches können einen suffizienten Abfluss kompromittieren. Letztere stellen eine definitive Kontraindikation zur weiteren VPS-Versorgung dar (Vinchon und Dhellemmes 2005). Dabei muss die lokal begrenzte Liquorpseudozyste klar von dem die gesamte Bauchhöhle betreffenden Aszites abgegrenzt werden. Erstmals wurde die Liquorpseudozyste als periumbilikalen Raumforderungen in Kombination mit Liquorshunts 1954 von Griffith Harsh (Harsh 1954) beschrieben. Die Zysten werden Pseudozysten genannt, weil die Zystenwand nur aus einer durch chronische inflammatorische Prozesse verdickten serösen Peritonealmembran besteht (Mobley et al. 2005) und nicht - wie bei herkömmlichen Zysten - epitheliales Gewebe das Innere auskleidet. Der genaue pathophysiologische Entstehungsprozess ist aktuell noch nicht vollständig geklärt. Es werden inflammatorische Prozesse und eine eventuelle Antigen-Antikörperantwort gegen das eingebrachte Fremdkörpermaterial oder die protein- und kohlenhydratreichen Bestandteile des einfließenden Liquors in Betracht gezogen. Prädisponierende Faktoren für die Entstehung sind abdominelle Voroperationen sowie multiple Shuntrevisionen, allergische Reaktionen auf Silikon oder Leberfunktionsstörungen (Hashimoto et al. 2004; Dabdoub et al. 2014). Die Prävalenzrate liegt bei 2,3\% und die jährliche Inzidenzrate ist in der Literatur mit 2,2\% angegeben.

Die Inzidenzzahlen über das Auftreten von abdominellen Komplikationen bei vorhandenem VPS sind schwierig zu erfassen. Die Datenlage hierfür ist unübersichtlich und die Zahlen schwanken deutlich zwischen 1,37\% und über 20\% (Vinchon und Dhellemmes 2005), Rush 
et al. (1985) geben eine Inzidenz von 15\% bei 300 Patienten an, welche über einen Zeitraum von zehn Jahren untersucht wurden, Chung et al. (2009) berichten von einer Inzidenz von 22,9\%. Weitere wissenschaftliche Arbeiten sprechen von Inzidenzraten bis $\mathrm{zu} 24 \%$ (Pumberger et al. 1998). Vinchon und Dhellemmes (2005) registierten bei der Untersuchung von 1564 Patienten mit einer Gesamt-Follow-up-Zeit von 16.689 Jahren 228 abdominellen Komplikationen. In der Tabelle 5 sind exemplarisch die Ergebnisse der empirischen Untersuchung dargestellt. Diese sind jedoch nur bedingt zur Vergleichbarkeit der Ergebnisse dieser Dissertation geeignet, da die in dieser Arbeit erfassten Parameter der stattgehabten abdominellen Voroperationen und die intraoperative Adhäsiolyse von Verwachsungen des Peritoneums nicht aufgezeichnet wurden.

Tabelle 5: Übersicht abdomineller Komplikationen

\begin{tabular}{|c|c|c|c|c|}
\hline Diagnose & Fallzahl & $\begin{array}{l}\text { Durchschnittsalter } \\
\text { bei Komplikation }\end{array}$ & Ursache (Fallzahl) & $\begin{array}{c}\text { Klin. Präsentation } \\
\text { (Fallzahl) }\end{array}$ \\
\hline Aszites & 13 & 3,4 Jahre & $\begin{array}{l}\text { Infektion (2) } \\
\text { Tumor (4) } \\
\text { Liquorüberproduk } \\
\text { tion (2) }\end{array}$ & $\begin{array}{l}\text { Bauchschmerzen (9) } \\
\text { ICP-Anstieg (1) } \\
\text { Infektion (3) }\end{array}$ \\
\hline Pseudozyste & 36 & 12,2 Jahre & $\begin{array}{l}\text { Infektion (15) } \\
\text { Aseptisch (21) }\end{array}$ & $\begin{array}{l}\text { Bauchschmerzen (21) } \\
\text { ICP-Anstieg (12) } \\
\text { Infektion (6) }\end{array}$ \\
\hline Darmperforation & 13 & 9,1 Jahre & $\begin{array}{l}\text { Intraoperativ (1) } \\
\text { Spätperforation } \\
\text { (12) }\end{array}$ & $\begin{array}{l}\text { Bauchschmerzen (3) } \\
\text { ICP-Anstieg (1) } \\
\text { Infektion (8) } \\
\text { Nephritis (1) }\end{array}$ \\
\hline Appendizitis & 47 & 9,9 Jahre & $\begin{array}{l}\text { Appendizitis (33) } \\
\text { ICP-Anstieg* (4) }\end{array}$ & $\begin{array}{l}\text { Appendizitis (34) } \\
\text { Peritonitis (13) }\end{array}$ \\
\hline $\begin{array}{l}\text { Hernien \& } \\
\text { Hydrozelen }\end{array}$ & 3 & 23,1 Jahre & $\begin{array}{l}\text { Hydrozele (12) } \\
\text { Leistenhernie (22) } \\
\text { Nabelhernie (2) } \\
\text { Schenkelhernie (1) }\end{array}$ & $\begin{array}{l}\text { Chirurgische } \\
\text { Versorgung war in } \\
\text { 24/32 Fällen } \\
\text { notwendig }\end{array}$ \\
\hline
\end{tabular}

* 4 Pat. mit ICP Anstieg und abd. Symp. aufgrund von Shuntversagen wurden irrtümlich appendektomiert Quelle: Vinchon \& Dhellmmes (2005) S.316 


\subsection{Komplikationen nach Frühgeburt}

Die Frühgeburtenrate liegt weltweit bei ca. 11,1\%. An den damit verbundenen Komplikationen sterben weltweit etwa 1 Million Kinder jährlich (Blencowe et al. 2013). Die Hauptprobleme in der direkten Phase nach der Frühgeburt sind die Entwicklung vom Atemnotsyndrom durch den Mangel an Surfactant, Nierenfunktionsstörungen und daraus folgende Elektrolytentgleisungen. Hinzu kommen die bereits erwähnten intrazerebralen Blutungen, ein eventuell persistierender Ductus arteriosus, Netzhauterkrankungen (Retinopathy of Prematurity), ein noch unreifes Immunsystems und die nekrotisierende Enterokolitis (Eichenwald und Stark 2008; Cutts et al. 2016). Darüber hinaus können langfristige Probleme wie beispielsweise neurologische Entwicklungsstörungen auftreten. Eine kurze Übersicht dazu bietet die Tabelle 6 des New England Journal of Medicine aus dem Jahre 2008.

Tabelle 6: Kurz- und langfristige Probleme bei Frühgeborenen

\begin{tabular}{|c|c|c|}
\hline $\begin{array}{l}\text { Betroffenes Organ } \\
\text { oder Organsystem }\end{array}$ & Kurzfristige Komplikationen & Langfristige Komplikationen \\
\hline Respiratorisches & $\begin{array}{l}\text { IRDS; Bronchopulmonale } \\
\text { Dysplasie, Luftverlust, Apnoe bei } \\
\text { Prämaturität }\end{array}$ & $\begin{array}{l}\text { Bronchopulmonale Dysplasie, } \\
\text { reaktive Atemwegserkrankung, } \\
\text { Asthma }\end{array}$ \\
\hline Gastrointestinales & $\begin{array}{l}\text { Hyperbilirubinämie, } \\
\text { Nahrrungsintoleranz, NEC, } \\
\text { Wachstumsstörung }\end{array}$ & $\begin{array}{l}\text { Kurzdarmsyndrom, } \\
\text { Gedeihstörung, Cholestase }\end{array}$ \\
\hline Immunologisches & $\begin{array}{l}\text { Krankenhaus-assoziierte- } \\
\text { Infektionen, Immundefizienz, } \\
\text { Perinatale Infektionen }\end{array}$ & RSV-Infektion, Bronchiolitis \\
\hline ZNS & $\begin{array}{l}\text { Intraventrikuläre Hirnblutung, } \\
\text { periventrikulären Leukomalazie, } \\
\text { Hydrozephalus }\end{array}$ & $\begin{array}{l}\text { Zerebrale Parese, } \\
\text { Hydrozephalus, Zerebrale } \\
\text { Atrophie, } \\
\text { Entwicklungsstörungen, } \\
\text { Gehörverlust }\end{array}$ \\
\hline Ophthalmologisches & Retinopathie der Frühgeborenen & $\begin{array}{l}\text { Sehverlust, Myopie, Strabismus, } \\
\text { Netzhauablösung }\end{array}$ \\
\hline Kardiovaskuläres & $\begin{array}{l}\text { Hypotension, PDA, Pulmonaler } \\
\text { Hypertonus }\end{array}$ & $\begin{array}{l}\text { Pulmonaler Hypertonus, } \\
\text { Bluthochdruck }\end{array}$ \\
\hline Nieren & $\begin{array}{l}\text { Elektrolytentgleisung, Störungen } \\
\text { des Säure-Basen-Haushalts }\end{array}$ & Bluthochdruck \\
\hline Hämatologisches & $\begin{array}{l}\text { Iatrogene Anämie, } \\
\text { Transfusionspflichtigkeit, } \\
\text { Anämie der Neugeborenen }\end{array}$ & \\
\hline Endokrines & $\begin{array}{l}\text { Hypoglykämien, } \\
\text { Schilddrüsenunterfunktion, } \\
\text { Kortisoldefizit }\end{array}$ & $\begin{array}{l}\text { Gestörte Glukosetoleranz, } \\
\text { erhöhte Insulinresistenz }\end{array}$ \\
\hline
\end{tabular}

Quelle: Eichenwald und Stark (2008) S.1701 


\subsection{Nekrotisierende Enterokolitis}

Die Erkrankung tritt bei ca. 7\% aller Frühgeborenen zwischen $500 \mathrm{~g}$ und $1500 \mathrm{~g}$ auf (Neu und Walker 2011). Die Überlebenswahrscheinlichkeit liegt bei ca. 70\% bis $80 \%$ und ist bei Kindern, die chirurgisch behandelt werden müssen, am geringsten (Fitzgibbons et al. 2009). Allerdings können auch Nicht-Frühgeborene Kinder von der Komplikation betroffen sein. Die Patientengruppe der Nicht-Frühgeborenen und der Spät-Frühgeborenen macht ca. 10\% aller nekrotisierenden Enterkolitiden aus und betrifft vor allem Kinder, die mit einem Herzfehler oder der Agangliose Morbus Hirschsprung zur Welt gekommen sind (Raboei 2009). Die genauen Pathomechachnismen der NEC sind zum jetzigen Zeitpunkt noch nicht genau geklärt, allerdings deutet vieles darauf hin, dass es sich um ein multifaktorielles Zusammenspiel von ätiologischen, mikrobiologischen und inflammatorischen Faktoren und fehlender Entwicklung des gastrointestinalen Traktes handelt (Ford et al. 1997; Neu 2014). Die daraus resultierende Darmschädigung führt zu einer Parese und Gewebsnekrosen. Die Hauptprädilektionsorte sind das terminale Ileum und der aufsteigende Kolonrahmen. Es kommt dabei zu Ansammlungen von Fäulnisgasen in der Darmwand (Pneumatosis intestinalis). Im schlimmsten Fall entwickelt sich eine Darmperforation mit daraus folgender Peritonitis bzw. Sepsis und einem möglichen letalem Ausgang (Neu und Walker 2011). Die Patienten zeigen dann Symptome wie Nahrungsverweigerung, diffuse Abwehrspannung der Bauchdecke und eventuell Blutstühle im Alter von 8-10 Tagen. Diagnostisch lässt sich Gewissheit erlangen, indem röntgenologisch oder per Sonografie gespannte Darmschlingen oder freie Luft im Abdomen nachgewiesen werden (Hollingsworth und Rice 2010; GarbiGoutel et al. 2014). Therapeutisch wird versucht, über einen möglichst langen Zeitraum konservativ zu behandeln, da verschiedene Studien gezeigt haben, dass sobald chirurgisch interveniert wird, sich das Outcome der Patienten signifikant verschlechtert (Blakely et al. 2005). Es gibt ebenso präventive Versuche, das Auftreten der NEC im Anfangsstadium zu verhindern. Dabei spielen Antibiotika, spezielle Ernährungsformen und pro- bzw. präbiotische Mikroorganismen eine wichtige Rolle. Der Erfolg oder Misserfolg dieser präventiven Methoden ist zum jetzigen Zeitpunkt noch nicht geklärt (Neu und Walker 2011; Bernardo et al. 2013; Asmerom et al. 2015). Die konservativen Behandlungsoptionen bei manifester nekrotisierender Enterokolitis beinhalten als Zielstellung eine abdominelle Dekompression. Dieses wird mithilfe von Nahrungskarenz und Umstellung auf parenterale Ernährung versucht und von Breitbandantibiose und intravenöser Flüssigkeitsgabe unterstützt. Im Falle von intestinaler Perforation, Schock oder Thrombozytenabfall ist eine sofortige chirurgische Operation indiziert. Diese beinhaltet dann die Anlage von Drainagen im Zusammenspiel mit einer explorativen Laparotomie mit oder ohne Resektion des 
betroffen Darmabschnitts und passagerem Anus präter (Neu und Walker 2011; Robinson et al. 2017). Allerdings kommt es postoperativ in bis zu 50\% der Fälle zu Komplikationen. Darin eingeschlossen sind Sepsis, Kurzdarmsyndrom, Wundinfektion, intraabdominelle Abszesse und Darmstenosen (Horwitz et al. 1995). Diese Prozesse können Verwachsungen und Vernarbungen begünstigen und im Falle eines VPS versorgten Hydrozephalus die notwendige peritoneale Liquorresorption kompromittieren. Dies trifft auch auf weitere abdominelle Operationen, wie beispielsweise nach Mekoniumileus oder Volvulus, zu (Kargl et al. 2017). Weiterhin konnten Mobley et al. (2005) in ihrer Studie über abdominelle Liquorpseudozysten bei liegendem VPS zeigen, dass 19\% der Patienten eine NEC in der Anamnese vorwiesen. 


\section{Patienten und Methoden}

\subsection{Versorgung von Kindern mit Hydrozephalus an der UMG}

Die Versorgung von Kindern mit angeborenem oder erworbenem Hydrozephalus wird in der Universitätsmedizin Göttingen durch einen multifaktoriellen, interdisziplinären Einsatz unterschiedlichster Fachdisziplinen umgesetzt. Dabei stehen die Kinderchirurgie, Kinderneurochirurgie, Neuropädiatrie und Neonatologie sowie Kollegen des Sozialpädiatrischen Zentrums stets in engem Kontakt. Das in Göttingen geschaffene Operative Kinderzentrum (OPKiZ) bildet mit der interdisziplinären Station 2023 die Plattform für eine optimale Versorgung der Kinder nach der neonatalogischen Phase. Dafür stehen Spezialsprechstunden, wie die Spina-bifida Sprechstunde oder das KINCHIHydrozephalus-Register, welches in Kapitel 2.5 noch weiter erläutert wird zur Verfügung. Moderne Ventil- und Shuntsysteme sowie aktuelle und Leitlinien-gerechte Operationsstandards bilden die Grundvoraussetzungen für die Behandlung der Patienten.

\subsection{Verwendete Shunts und Ventile an der UMG}

Die an der Universitätsmedizin Göttingen verwendeten Shuntsysteme und Ventiltypen sind für den Überblick in der Tabelle 7 aufgeführt. Die Auswertung und Bewertung der Fälle nach verwendeter Modellkombination ist nicht Gegenstand dieser Dissertation.

Über den Untersuchungszeitraum wurden verschiedene, zu den jeweiligen Zeiten verfügbare Ventilsysteme verwendet. Grundsätzlich sind fixe Differentialdruckventile, später programmierbare Differentialdruckventile und dann bis heute programmierbare Gravitations-assistierte Ventile und programmierbare Shuntassistenten verwendet worden. Der gegenwärtige Standard ist ein fixes, nicht adaptierbares Differentialdruckventil (miniNAV) mit nachgeschaltetem, programmierbaren, Gravitations-assistierten Shuntassistenten (proSA). 
Tabelle 7: Übersicht Shunthardware

\begin{tabular}{|l|l|l|}
\hline Proximale Katheter & Distale Katheter & Ventiltyp \\
\hline Miethke & Miethke & proSA (Miethke) \\
\hline Bioglide & Bioglide & PS Medical (MD) \\
\hline Miethke & Miethke & Medos prog. (Codman) \\
\hline Bactiseal & Bactiseal & proGAV (Miethke) \\
\hline
\end{tabular}




\subsection{VPS-Neuanlage - Standardprozedere an der UMG}

Das operative Vorgehen zur Anlage eines VP Shunts ist im nachfolgenden Text in den Grundzügen erläutert.

Der Patient wird in Rückenlage in Intubationsnarkose mit dem Kopf auf einem Gelring gelagert, die rechte Schulter unterpolstert, es erfolgt die Kopfwendung nach links. Die Operation beginnt mit der Rasur des Operationsgebiets entlang des geplanten Shuntverlaufs und dem Anzeichnen der bogenförmigen Schnittführung rechts frontal sowie der kurzen geraden Schnitte parietal und paraumbilikal rechts. Es wird steril abgewaschen und abgedeckt. Nach einem Handschuhwechsel und dem Aufbringen einer sterilen Folie werden die markierten Bereiche mit isotonischer Natriumcloridlösung unterspritzt. Im ersten Schritt wird der Schädel trepaniert und die intakte Dura dargestellt. Als nächster Punkt wird die parietale subkutane Tasche für das spätere Implantat vorbereitet, bevor paraumbilikal die Eintrittspforte ins Peritoneum präpariert wird. Mit dem Untertunnelungsmandrin wird eine Verbindung zum Durchziehen des Peritonealkatheters nach paraumbilikal geschaffen. Über einen Trocar kann nun die zuvor inzidierte Muskelfascie penetriert und der Kunststoffkatheter bis zu einer Länge von $90 \mathrm{~cm}$ widerstandslos eingeführt werden. Mit einer Wasserprobe wird die suffiziente Durchgängigkeit und leichte Instillation in den Peritonealraum geprüft. Nun erfolgt am Kopf die Durainzision und das Einführen des Ventrikelkatheters in eine Tiefe von 5,5-6,0 cm in Projektion auf das Vorderhorn des ipsilateralen Seitenventrikels, bis spontan Liquor entweicht. An diesem Punkt der Operation kann der Ventrikelkatheter mit dem Ventil konnektiert werden und subkutan nach parietal durchgezogen werden. Dort und beidseits lateral des Bohrlochs kann das System mit nicht resorbierbaren Nähten am Periost fixiert werden. Im letzten Schritt wird der distale Teil des Katheters mit dem zentralen Teil verbunden und ein Funktionstest durchgeführt. Lässt sich das Reservoir problemlos pumpen und füllen, wird nochmals die Hämostase kontrolliert, bevor der Wundverschluss erfolgt. Hierzu werden engmaschige resorbierbare Subkutannähte verwendet. Der Hautverschluss erfolgt durch Leukostrips, die mit einem sterilen Pflasterverband gedeckt werden. Mit dem Umlagern des Patienten ist die Operation beendet. 


\subsection{Datenerfassung und Selektion des Patientenkollektivs}

Mithilfe des Kinderneurochirurgischen Hydrozephalus \& Shunt Registers wurden aus über 500 registrierten Patienten mit Hydrozephalus primär alle Patienten mit Shunt-versorgtem Hydrozephalus selektioniert. Von diesem Patientenkollektiv wurde ein entsprechender Datensatz mit insgesamt 1184 korrespondierenden Shunt-Operationen erstellt. Im nächsten Schritt erfolgte die Exklusion aller operativen Datensätze außerhalb des Untersuchungszeitraums und die weitere Selektion ausschließlich nach Shunt-RevisionsOperationen. Danach wurden dann zusätzlich alle Operationen ausgeschlossen, welche außerhalb der UMG durchgeführt wurden und/oder keine Revision des abdominellen Katheterschenkels einbezogen. Schließlich konnten 61 interdisziplinär absolvierte abdominelle Katheter-Revisionen zur weiteren Auswertung herangezogen werden. Die Abbildung 1 veranschaulicht diesen Prozess. Deutlich wird in dieser Grafik auch, dass die Mehrzahl aller Shuntrevisionen mit Peritonealkatheterrevision nämlich 312 Fälle ohne interdisziplinäre Zusammenarbeit versorgt werden konnten.

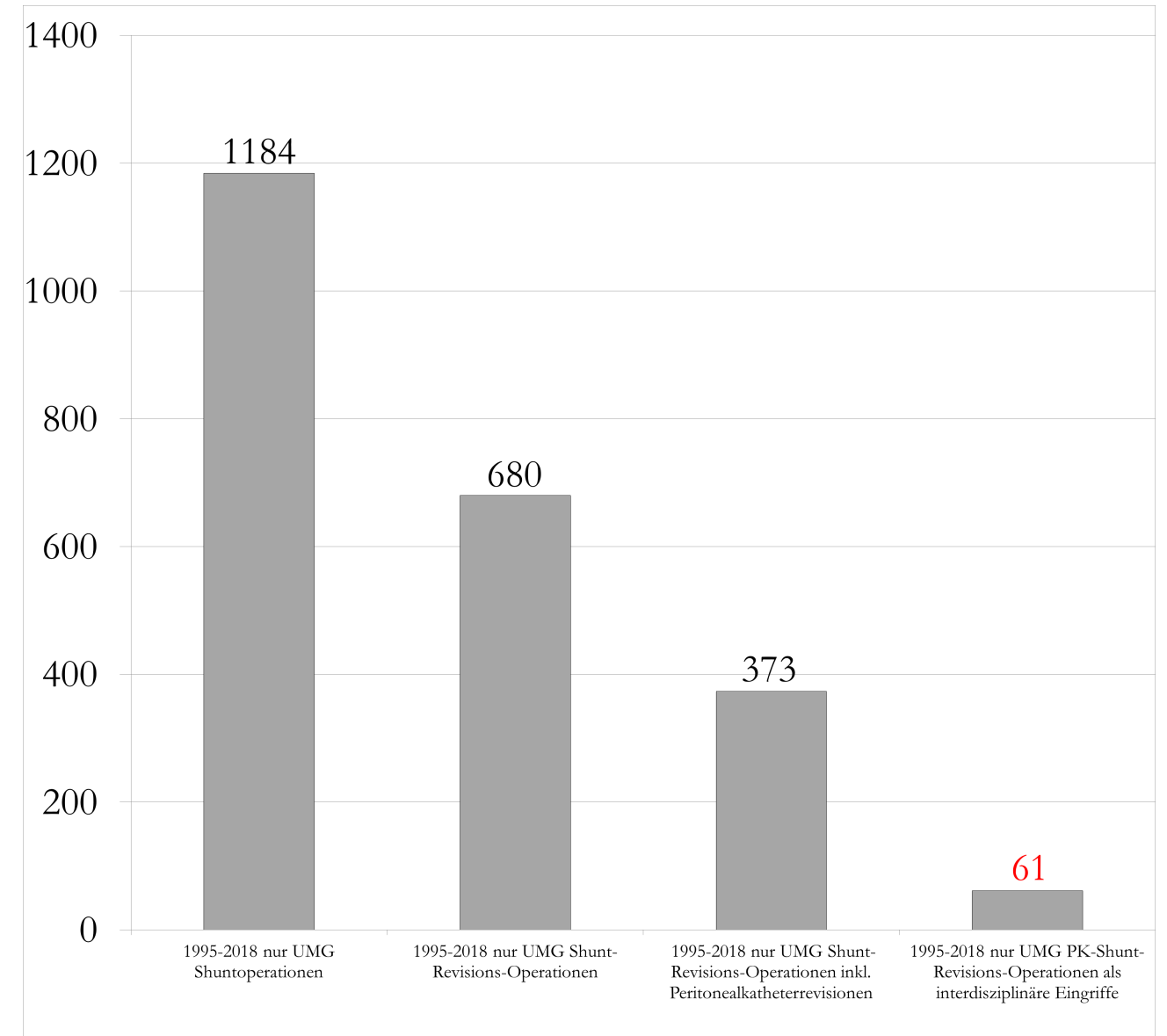

Abbildung 1: Auswahl des Patientenkollektivs 
Die Auswahl erfolgte mit Zugriff auf die Datenbank des Göttinger Shuntregisters, welches in Kapitel 2.5 näher erläutert wird. Die erhobenen Daten wurden in eine Tabelle aufgenommen und anschließend unter Einbezug von Arztbriefen und Fallakten aller Fachdisziplinen, Operationsberichten, radiologischen und mikrobiologischen Befunden ergänzt. Dafür verwendet die UMG Göttingen die Systeme iXServ der OSM Gruppe und PACS (Picture Archiving and Communication System) für die radiologischen Befunde. Die Akteneinsicht erfolgte ausschließlich an dem für Doktoranden eingerichteten Arbeitsplatz der Station 2023 (Operatives Kinderzentrum: Universitätsmedizin Göttingen). 


\subsection{Göttinger Shuntregister}

Die besondere Grundlage dieser Arbeit ist das Hinzuziehen des Göttinger Shuntregisters, welches seit 1995 strukturiert Übersicht über den Behandlungsverlauf aller pädiatrischer Shuntpatienten an der Universitätsmedizin Göttingen gibt. Es handelt sich dabei um ein prospektives Register, welches das Krankheitsbild des kindlichen Hydrozephalus multifaktoriell erfasst und dem behandelnden Arzt in kürzester Zeit ohne weitere Rückfragen oder Akteneinsicht alle relevanten Information zur Behandlung zur Verfügung stellt. Neben chirurgischen Informationen werden auch der neurologische Status des Kindes und ein Quality-of-Life-Score erfasst, welcher Aufschluss über den langfristigen Therapieerfolg geben kann. In Zeiten der Digitalisierung und immer umfassenderen klinisch erfassten Daten ist die Einführung des Registers mit Einbindung in den klinischen Alltag der konsequente Schritt zur Bündelung und Ordnung der Informationen. Weiterhin können Daten spezifisch extrahiert werden und ermöglichen somit die Schnittstelle zur fundierten wissenschaftlichen Aufarbeitung. Zum jetzigen Zeitpunkt (Stand Oktober 2017) wurden 579 Hydrozephalus Patienten im System registriert mit dazugehörigen 1874 chirurgischen Interventionen. ${ }^{1}$ Das mediane Follow-up der Patienten liegt bei 8,5 Jahren. Dabei erfolgt die Sammlung der Daten streng nach dem deutschen Datenschutzgesetz und ist unabhängig vom zuständigen Datenbeauftragen geprüft worden. Zu keinem Zeitpunkt des Entwicklungsprozesses und darüber hinaus erfolgte ein Sponsoring von industrieller Seite, um mögliche Interessenkonflikte zu vermeiden.

1 Anm.: Die Datengrundlage dieser Dissertation bilden die im Register gesammelten Informationen von 19952018 


\subsection{Erfasste Parameter der Datenauswertung}

In Tabelle 8 sind die erhobenen Parameter aufgelistet, die im nächsten Schritt Eingang in die statistische Auswertung fanden.

Tabelle 8: Untersuchungsparameter

\begin{tabular}{|c|c|}
\hline & Untersuchungsparameter \\
\hline 1. & Geschlecht \\
\hline 2. & Alter zum Zeitpunkt der Komplikation in Jahren \\
\hline 3. & Letzte Follow-up Untersuchung \\
\hline 4. & Datum der interdisziplinären Komplikation \\
\hline 5. & Zeitraum nach interdisziplinärer Komplikation in Tagen \\
\hline 6. & Dringlichkeit des Eingriffs \\
\hline 7. & Ätiologie des Hydrozephalus \\
\hline 8. & Operationsindikation aus Neurochirurgischer Sicht \\
\hline 9. & Operationsindikation aus Kinderchirurgischer Sicht \\
\hline 10. & Eventueller Z. n. nekrotisierender Enterokolitis \\
\hline 11. & Frühgeborenenstatus \\
\hline 12. & Frühgeborenenstatus in SSW \\
\hline 13. & Geburtsgewicht in Gramm \\
\hline 14. & Blutungsgrad nach Lou Ann Papile I bis IV \\
\hline 15. & Infektion des Shuntsystems \\
\hline 16. & Eventueller Keimnachweis \\
\hline 17. & Ableitungsart des Shuntsystems nach Intervention \\
\hline 18. & Operativer Zugang zum distalen Katheter \\
\hline 19. & Anzahl der Gesamtshuntrevisionen \\
\hline
\end{tabular}




\subsection{Interdisziplinäre Datenaufbereitung}

Zusätzlich zu der im Kapitel 2.4 beschriebenen Datenextrahierung als Grundlage der Dissertation ist insbesondere die interdisziplinäre Aufarbeitung der Daten von Seiten der Neurochirurgen und Kinderchirurgen zum doch sehr speziellen Patientenkollektiv hervorzuheben. Im kritischen Dialog wurden die Informationen zum Beispiel zur Operationsindikationsstellung oder abdominellen Komplikation selektiert. Dabei war es erforderlich die Aussagefähigkeit der zu klassifizieren Daten zu prüfen gemeinsam Interpretationsmöglichkeiten und Grenzen festzulegen.

\subsection{Statistische Auswertung}

Die statistische Auswertung der erhobenen Daten erfolgte nach Aufnahme der Daten durch das Programm Microsoft Excel (Version 15.17 für Mac) mit dem Programm SPSS Statistics (Version 24.0 für Mac) der Firma IBM. Hierfür wurde die Excel-Tabelle in eine SPSS-fähige Form überführt und bestimmte erfasste Parameter dichotomisiert. Hieraus ergab sich ein aus 61 Fällen bestehender Datensatz mit 58 Variablen. Anschließend wurden die Daten durch deskriptive Statistik beschrieben und über die Ausgabefunktion visualisiert. Dabei wurden relative und absolute Häufigkeiten erfasst sowie die Angaben für Mittelwert, Standardabweichung, Median und Streuung der metrisch skalierten Daten aufgezeichnet. Auf diesem Weg wurden die Altersverteilung und das Geschlecht sowie die OP-Indikationen genau erfasst. Zur Veranschaulichung der Daten wurden Balkendiagramme, Kreisdiagramme, Kreuztabellen und Tabellen verwendet.

Aufgrund der Selektion des Patientenkollektivs und der daraus resultierenden geringen Fallzahl war eine valide statistische Auswertung erschwert. In diesem Zusammenhang sei darauf hingewiesen, dass die gewonnenen Ergebnisse zu jeder Zeit kritisch zu hinterfragen sind und allenfalls als Tendenz angesehen werden können. 


\subsection{Datenschutzkriterien und Literaturrecherche}

Die Auswertung der Daten erfolgte nach den strengen Regeln des Datenschutzes und nach bestem Wissen und Gewissen. Der Ethikantrag für das dieser Dissertation zugrunde liegende Datenregister wurde durch die Ethikkommission der Universitätsmedizin Göttingen bewilligt. (12/9/2017)

Begleitend zum Arbeitsprozess wurde eine intensive Literaturrecherche durchgeführt. Dabei wurden vorwiegend die elektronischen Datenbanken von Medline und National Library of Medicine über den HAN-Server der Staats- und Universitätsbibliothek Göttingen genutzt. Die wesentlichen Suchparameter waren hierbei Folgende: hydrocephalus, infant, shunt, complication, abdominal oder VPS. Zur Quellendokumentation wurde das Programm Zotero verwendet. 


\section{Ergebnisse}

\subsection{Darstellung des Patientenkollektivs}

\subsubsection{Geschlechtsverteilung}

In der vorliegenden Studie wurden die Daten von 44 Patienten ausgewertet, welche in 61 Fällen interdisziplinär von der Neurochirurgie und der Klinik für AllgemeinViszeral/Kinderchirurgie der Universitätsmedizin Göttingen betreut wurden. Von den 44 Patienten waren 19 weiblich (43\%) und 25 männlich (57\%). Es ergeben sich 26 Fälle (43\%), bei denen weibliche Patientinnen interdisziplinär behandelt wurde und 35 Fälle (57\%), bei denen dies auf männliche Patienten zutrifft.

\subsubsection{Altersverteilung der interdisziplinären Fälle}

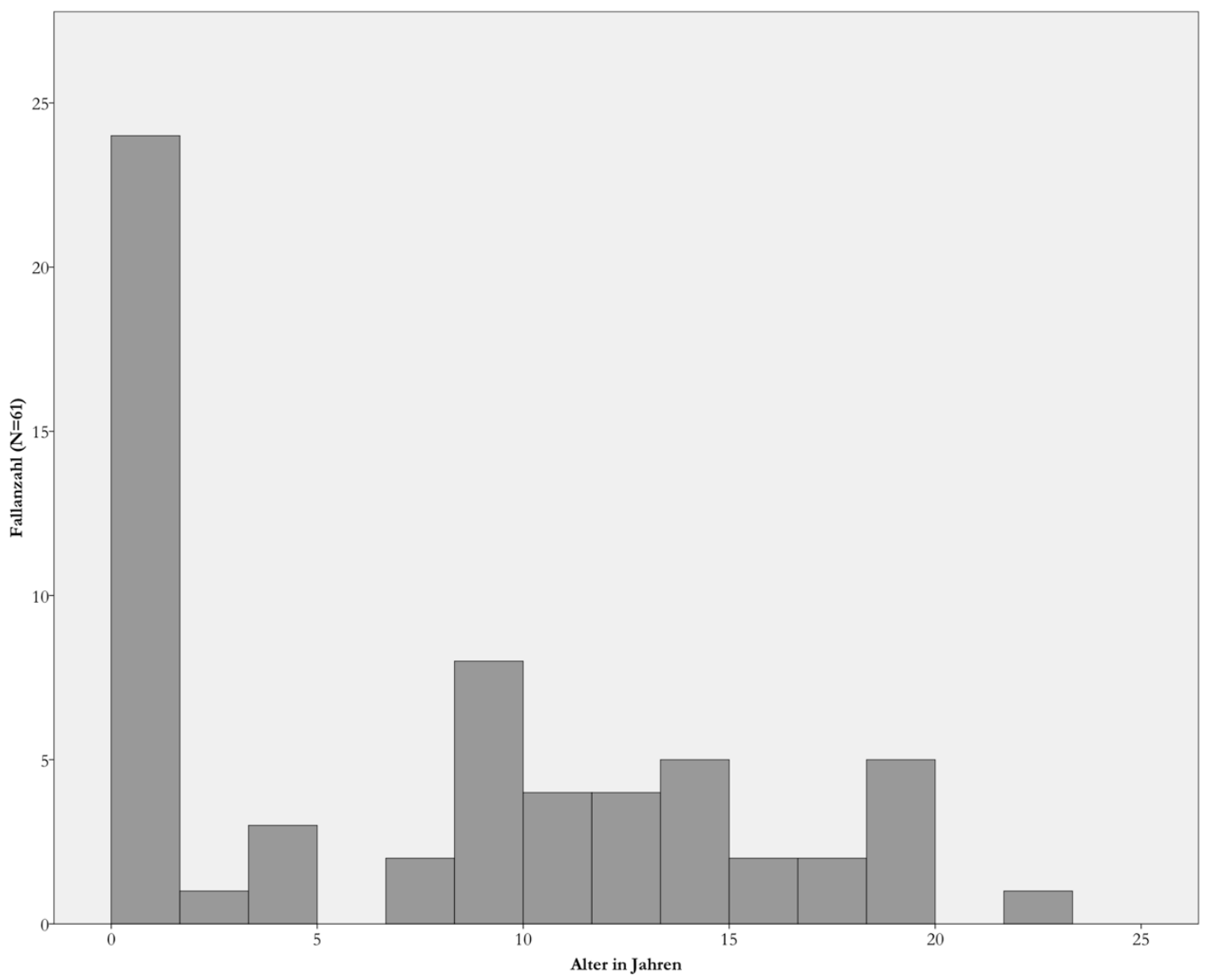

Abbildung 2: Alter zum Zeitpunkt der interdisziplinären Versorgung in Jahren

In Abbildung 2 ist ein Überblick über die Altersverteilung zum Zeitpunkt der interdisziplinären Versorgung gegeben. Es zeigt sich, dass gerade im ersten Lebensjahr 
gehäuft Fälle auftreten. Frühgeborenen bedürfen oft einer interdisziplinären Versorgung. Die anderen Fälle sind homogen verteilt.

\subsubsection{Anzahl der gesamten Shuntrevisionen pro Patient}

Tabelle 9: Deskriptive Statistik der gesamten Shuntrevisionen pro Patient

\begin{tabular}{|l|c|}
\hline \multicolumn{1}{|c|}{ Wert } & Anzahl \\
\hline Mittelwert & 3,3 \\
\hline Median & 3 \\
\hline Standardabweichung & 2,8 \\
\hline Spannweite & 13 \\
\hline Minimum & 0 \\
\hline Maximum & 13 \\
\hline
\end{tabular}

Die Tabelle 9 gibt einen Überblick über die Anzahl der Shuntrevisionen, die mit jedem Patienten verknüpft sind. Im Durchschnitt wird jeder Patient im Verlauf dreimal aufgrund einer Revision des Shunts operativ versorgt. Die Spannweite beträgt 13 Revisionsoperationen bei einer Standardabweichung von 2,8. Es konnten die Werte von allen Patienten erfasst werden. 


\subsection{4 Ätiologie der Hydrozephali}

\section{Ätiologie der Hydrozephali}

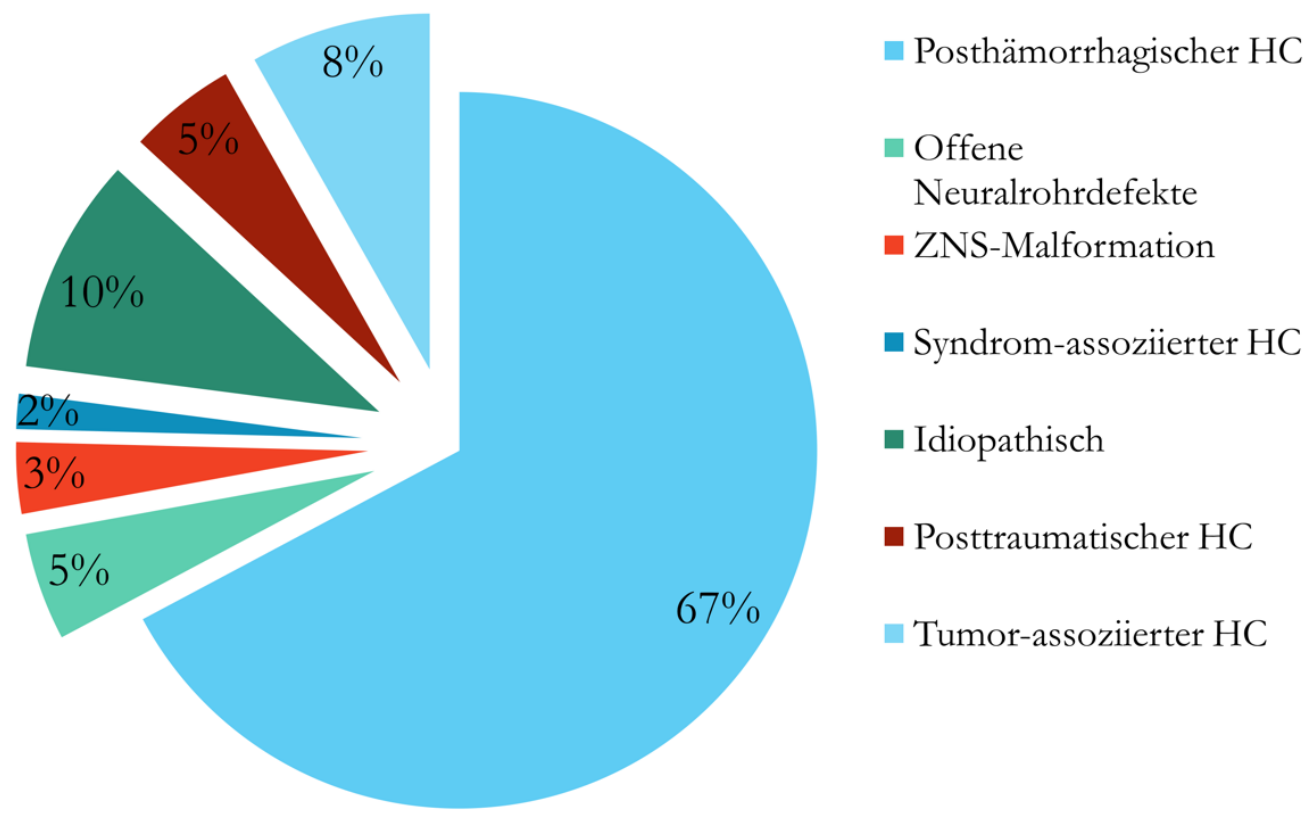

Abbildung 3: Ursachen des kindlichen Hydrozephalus im Überblick

In Abbildung 3 werden die Ursachen für das Vorhandensein des Hydrozephalus grafisch dargestellt. Es zeigte sich im untersuchten Kollektiv hierbei sehr deutlich, dass der Entstehung des Hydrozephalus in 67\% der Fälle eine intraventrikuläre Hirnblutung mit daraus folgendem posthämorrhagischen Verschluss zugrunde lag. Die anderen ätiologischen Ursachen befinden sich im Prozentbereich zwischen 2\% und 10\%. 


\subsection{Potentielle Risikofaktoren für abdominelle Shuntkomplikationen}

\subsubsection{Einflussgröße Frühgeburtlichkeit}

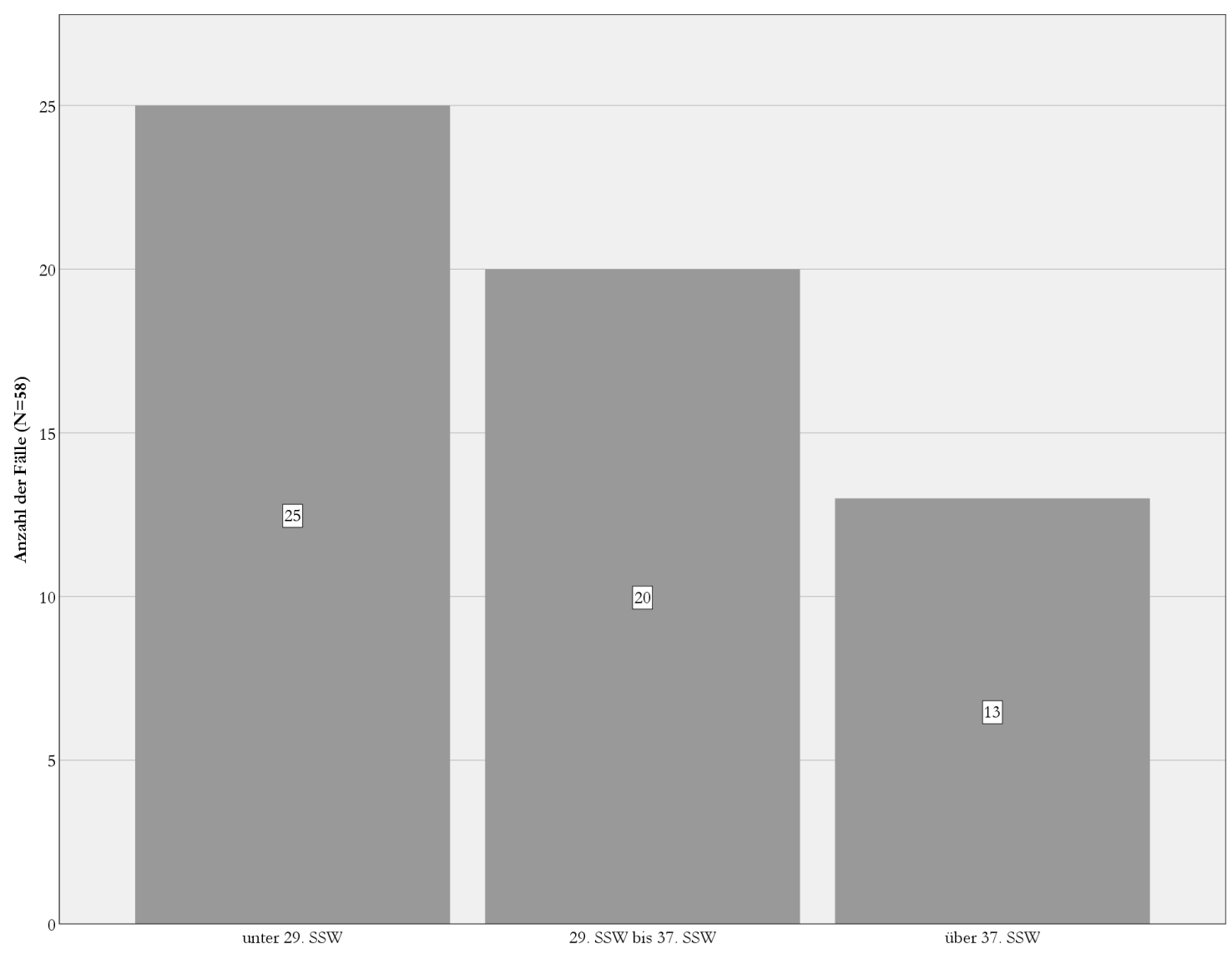

Abbildung 4: Einteilung des Fallkollektivs nach Schwangerschaftswoche

Im gesamten Fallkollektiv wurde in 48 der 61 Fälle der Faktor Frühgeburtlichkeit erfüllt d. h. vor der vollendeten 37. Schwangerschaftswoche (Abk. SSW) entbunden. Es sind somit 79\% der Fälle positiv für den eventuellen Risikofaktor Frühgeburtlichkeit. In 3 Fällen des Kollektivs konnte zwar die Frühgeburtlichkeit bestätigt, jedoch nicht die genaue Schwangerschaftswoche angegeben werden, diese Werte finden somit keinen Eingang in die Statistik. In Abbildung 4 ist die Häufigkeitsverteilung unter dem Gesichtspunkt der Schwangerschaftswoche abgebildet. In Tabelle 10 sind die 45 Fälle mit Frühgeburtlichkeit noch einmal spezifisch deskriptiv beschrieben. Bei den diagnostizierten Frühgeburten zeigte sich eine Streuung zwischen der 23. und der 36. Schwangerschaftswoche. Es traten bei Mädchen und Jungen gehäuft Fälle um die 24. und 25. SSW auf. Im Durchschnitt kamen die Kinder in der 28. Schwangerschaftswoche zur Welt. 
Tabelle 10: Deskriptive Statistik der Frühgeburtlichkeit in SSW

\begin{tabular}{|l|c|}
\hline \multicolumn{1}{|c|}{ Wert } & SSW \\
\hline Mittelwert & 28 \\
\hline Median & 27 \\
\hline Standardabweichung & 3,8 \\
\hline Spannweite & 13 \\
\hline Minimum & 23 \\
\hline Maximum & 36 \\
\hline
\end{tabular}

\subsubsection{Risikofaktor Geburtsgewicht}

Tabelle 11: Deskriptive Statistik des Geburtsgewichts der Frühchen

\begin{tabular}{|l|c|}
\hline \multicolumn{1}{|c|}{ Wert } & Gewicht $[\mathrm{g}]$ \\
\hline Mittelwert & 1033 \\
\hline Standardabweichung & 807 \\
\hline Minimum & 550 \\
\hline Maximum & 3450 \\
\hline Median & 720 \\
\hline
\end{tabular}

Tabelle 11 zeigt einen Überblick über die Geburtsgewichte der Frühgeboren. Von den 48 Frühgeborenen konnte in 33 Fällen das Geburtsgewicht festgestellt werden. In 15 Fällen konnten keine Angaben über das Gewicht gemacht werden, obwohl eine Frühgeburtlichkeit vorlag. Bei den 13 reif geborenen Kindern wurde das Gewicht nicht erfasst. Die Datenlage ist auch aufgrund der erst seit 2008 im IXServ in digitaler Form vorliegenden Informationen nicht besser darstellbar. Auffällig war jedoch die sehr große Spannweite von $550 \mathrm{~g}$ bis $3450 \mathrm{~g}$ Geburtsgewicht. Im Durchschnitt wogen die Kinder 1033g. 


\subsubsection{Untersuchung zum Einteilungsgrad der intraventrikulären Blutungen}

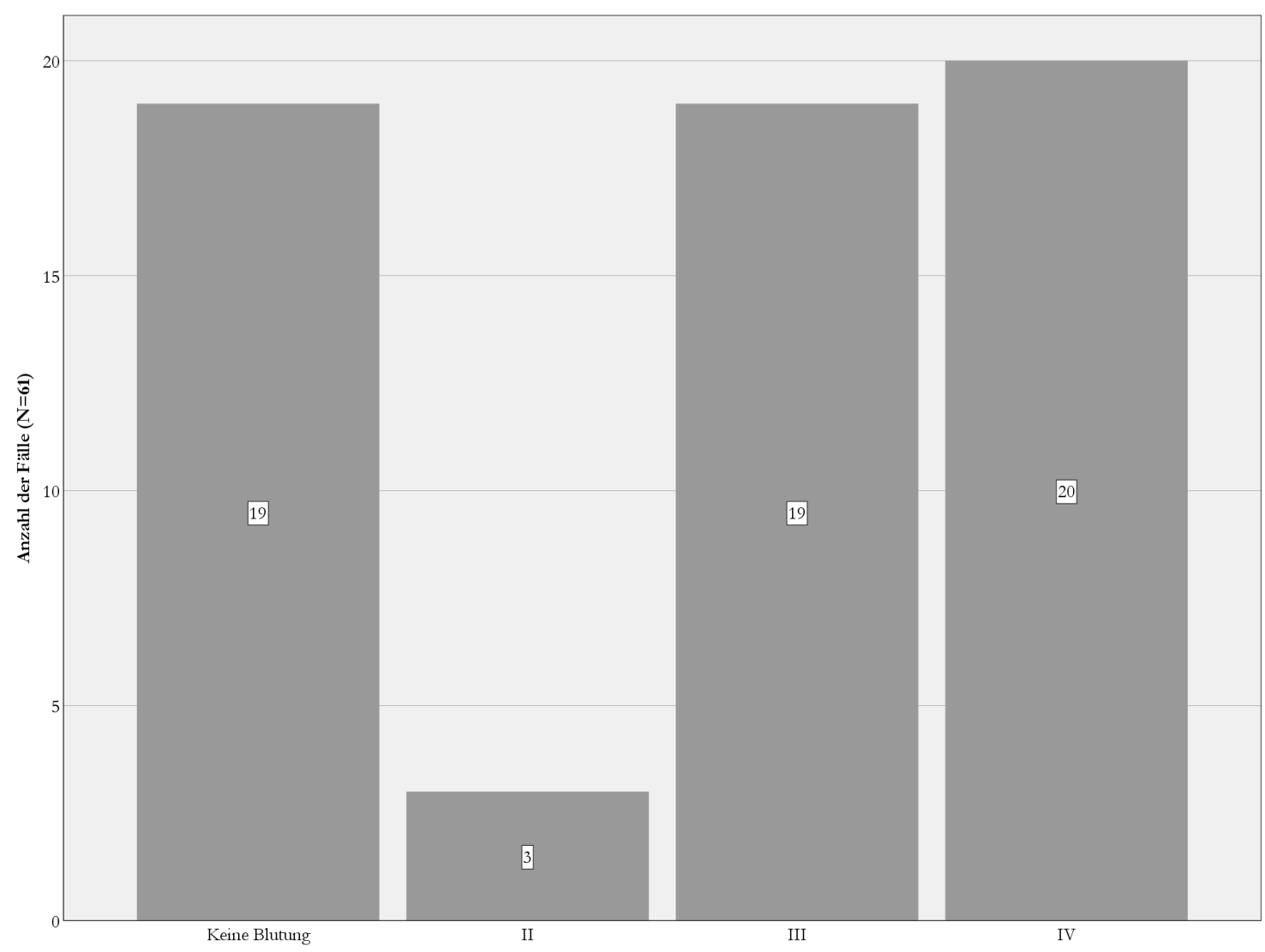

Abbildung 5: Darstellung des Blutungsgrads nach Lou Ann Papile

In der vorliegenden Studie wurden die Fälle auch auf das Vorliegen von intraventrikulären Hirnblutungen untersucht. Dazu wurde die Einteilung nach Lou Ann Papile von Grad I IV verwendet, da diese zum Zeitpunkt des Beginns der Datenerhebung aktuell war. Die Abbildung 5 gibt einen geordneten Überblick über den Schweregrad der intraventrikulären Hirnblutung. In 42 Fällen (69\%) lag eine Blutung vor. Dabei ist die Mehrzahl der Fälle (64\%) mit Blutungen des Grades III oder IV assoziiert. In insgesamt 19 Fällen (31\%) lag keine Blutung vor. 


\subsubsection{Frühgeborenenstatus und nekrotisierende Enterokolitis}

Tabelle 12: Kreuztabelle Frühgeborenenstatus * NEC

\begin{tabular}{|c|l|c|c|c|}
\hline \multicolumn{2}{|c|}{} & \multicolumn{2}{c|}{ Z. n. Nekrotisierender Enterokolitis } & \multirow{2}{*}{ Gesamt } \\
\cline { 2 - 4 } & Nein & Ja & 13 \\
\hline \multirow{2}{*}{ Frühgeborenen } & Nein & 13 & 0 & 48 \\
\cline { 2 - 4 } & $\mathrm{Ja}$ & 25 & $\mathbf{2 3}$ & 61 \\
\hline \multicolumn{2}{|c|}{ Gesamt } & 38 & 23 & \\
\hline
\end{tabular}

In Tabelle 12 ist der Zusammenhang zwischen Frühgeburt und dem Auftreten der nekrotisierenden Enterokolitis (NEC) verdeutlicht, da diese als ein Einflussfaktor für das Auftreten von abdominellen Folgekomplikationen vermutet wird. Hieraus lässt sich erkennen, dass von den 48 Fällen von Frühgeburt 23 Fälle (38\%) mit dem Auftreten der Erkrankung assoziiert sind.

\subsubsection{Infektion und Keimnachweis}

Tabelle 13: Kreuztabelle zur Darstellung von Infektion und Keimnachweis

\begin{tabular}{|c|c|c|c|c|}
\hline & \multicolumn{2}{|c|}{ Infektion des Shuntsystems } & \multirow[b]{2}{*}{ Gesamt } \\
\hline & & Nein & $\mathrm{Ja}$ & \\
\hline \multirow[t]{2}{*}{ Keimnachweis } & Nein & 51 & 4 & 55 \\
\hline & $\mathrm{Ja}$ & 0 & 6 & 6 \\
\hline \multicolumn{2}{|l|}{ Gesamt } & 51 & 10 & 61 \\
\hline
\end{tabular}

In der Tabelle 13 wird deutlich, dass in 10 Fällen (16\%) der interdisziplinären Behandlung eine Infektion des Shuntsystems vorlag. In 6 Fällen (10\%) war hierbei ein Keimnachweis möglich, in 4 Fällen (7\%) war dies nicht möglich. Die bei einem Erregernachweis gefundenen Bakterien waren die grampositiven, fakultativ aeroben Kokken Staphylococcus aureus, Staphylococcus epidermidis, Streptococcus viridans, Streptococcus pyogenes sowie das grampositive anaerobe Bakterium Propionibacterium acnes. 


\subsection{Indikationsstellung zur interdisziplinären Shuntrevision}

\subsection{1 Übersicht der Operationszugänge zum distalen Teil des VPS}

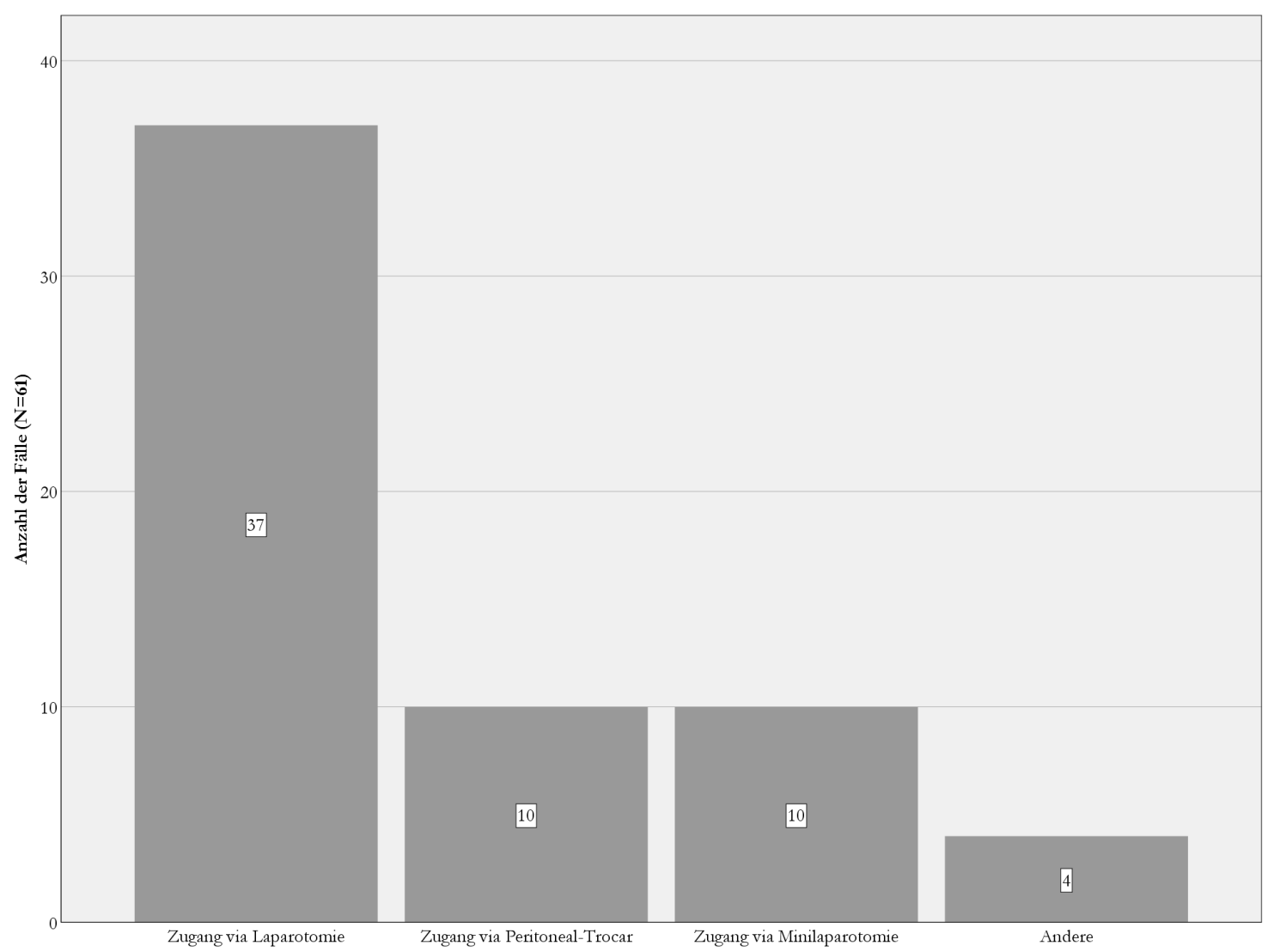

Abbildung 6: Einteilung des operativen Zugangswegs zum distalen Katheter

In Abbildung 6 sind die operativen Zugangswege zum distalen Ende des Shuntsystems während der interdisziplinären Versorgung, gewichtet nach Häufigkeit, dargestellt. Die Laparotomie stellt mit 37 Fällen (61\%) den häufigsten Zugangsweg dar. An zweiter Stelle steht der endoskopische Zugang über einen Peritoneal-Trocar mit einer Gesamthäufigkeit von 10 Fällen (16\%). Als weiterer Zugangsweg wurde zehn Mal (16\%) die Minilaparotomie durchgeführt. In 4 Fällen (7\%) wurden alternative Zugangswege gewählt. 


\subsubsection{Ableitungsart nach interdisziplinärer Versorgung}

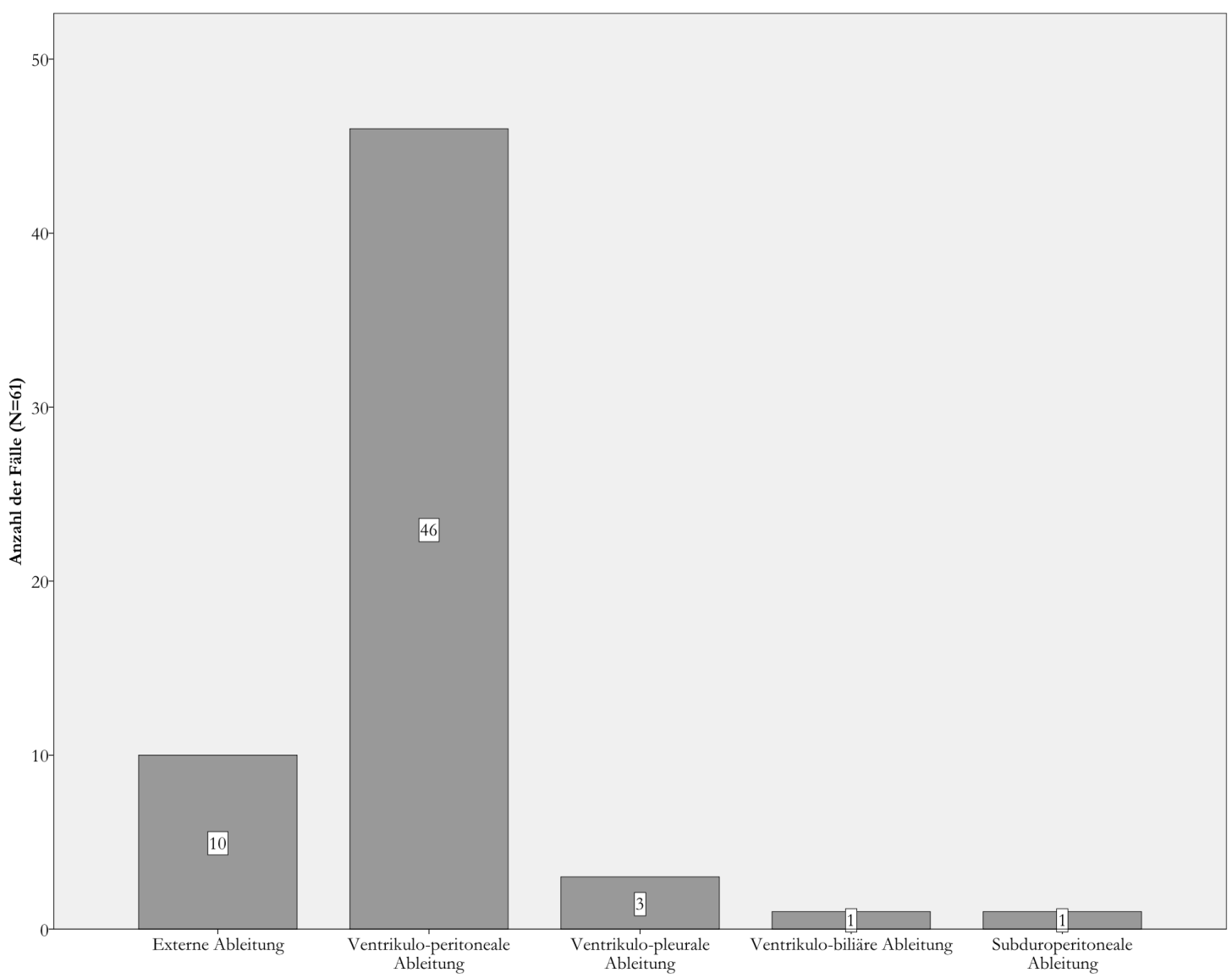

Abbildung 7: Ableitungsart des Shunts postinterventionell

Die Abbildung 7 zeigt die Art der Shuntversorgung nach stattgefundener interdisziplinärer Versorgung. Die beiden erstgenannten Punkte repräsentieren $92 \%$ aller Fälle, wohingegen die restlichen drei Ableitungsmöglichkeiten eher selten angewendet wurden. Hierbei wurde das liegende VPS Shuntsystems revidiert und dann alternativ nach den oben genannten Möglichkeiten abgeleitet. Dabei muss die Zusammenarbeit mit den Kollegen der Herz-, Thoraxchirurgie zur Anlage der ventrikulo-pleuralen Shuntsysteme angemerkt werden. 


\subsubsection{Dringlichkeit der Operation}

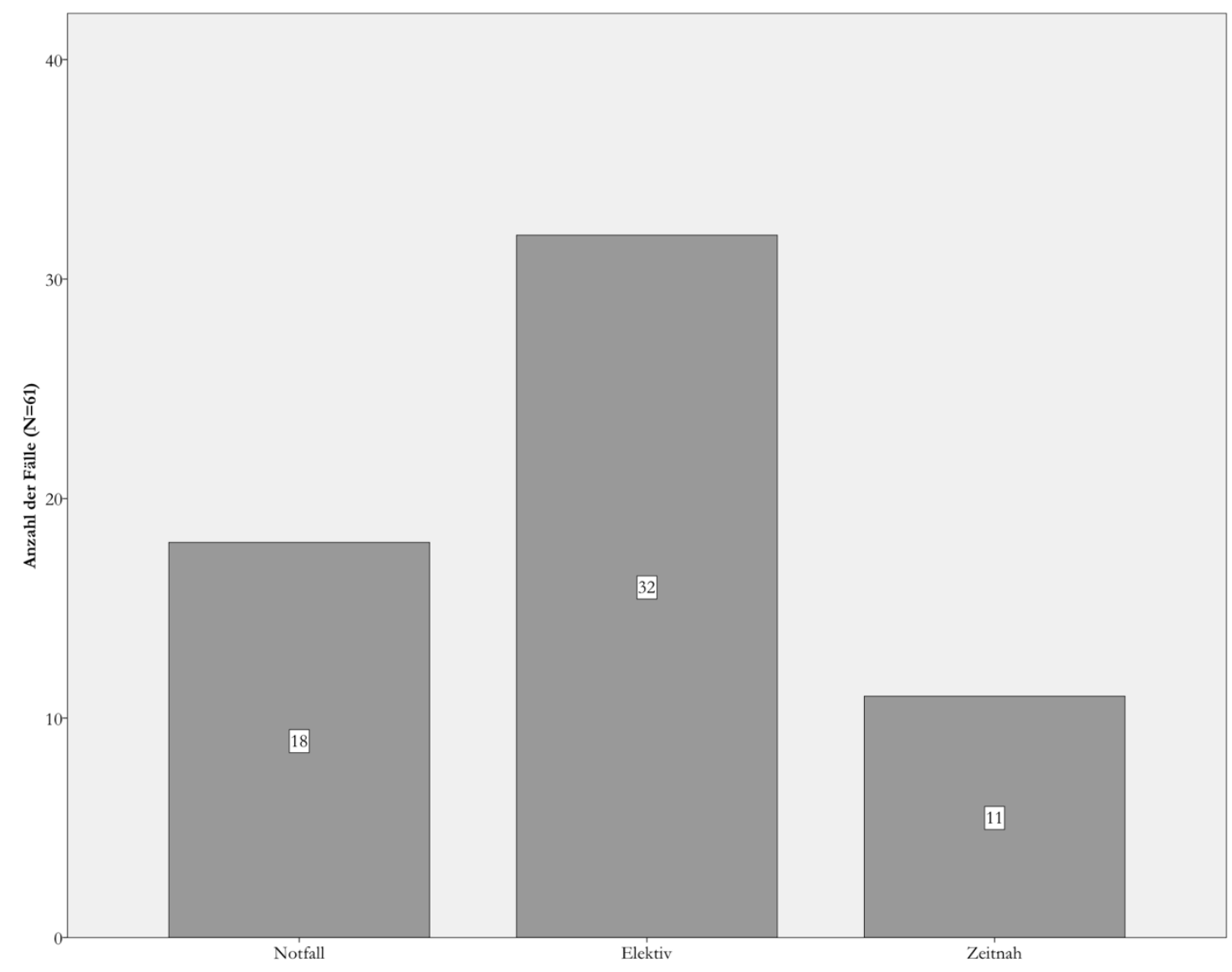

Abbildung 8: Notwendigkeit des Eingriffs

Die Abbildung 8 gibt einen Überblick über die Dringlichkeit mit der die interdisziplinäre Behandlung erfolgt ist. In 32 der Fälle (52\%) waren die gemeinsamen Operationen elektiv gelegt und planbar. In 18 Fällen (30\%) musste notfallmäßig interdisziplinär interveniert werden. Elf Fälle (18\%) ordneten sich mit der Eintragung der zeitnahen Versorgung zwischen notfallmäßiger Indikation und geplanter Versorgung als dringlich ein. 


\subsubsection{Operationsindikation aus neurochirurgischer Sicht}

Tabelle 14: Neurochirurgische Behandlungsindikationen

\begin{tabular}{|c|c|c|c|}
\hline & & $\mathrm{N}$ & Prozent \\
\hline \multirow[t]{6}{*}{ Indikation } & Anlage eines ventrikulo-peritonealen Shuntsystems & 21 & $34,4 \%$ \\
\hline & $\begin{array}{l}\text { Explantation des Shuntsystems und externe } \\
\text { Ventrikeldrainage }\end{array}$ & 10 & $16,4 \%$ \\
\hline & Revision des Peritonealkatheters & 25 & $41 \%$ \\
\hline & Anlage eines ventrikulo-pleuralen Shuntsystems & 3 & $5 \%$ \\
\hline & Anlage eines ventrikulo-biliären Shuntsystems & 1 & $1,6 \%$ \\
\hline & Anlage eines subduroperitonealen Shuntsystems & 1 & $1,6 \%$ \\
\hline \multicolumn{2}{|l|}{ Gesamt } & 61 & $100,0 \%$ \\
\hline
\end{tabular}

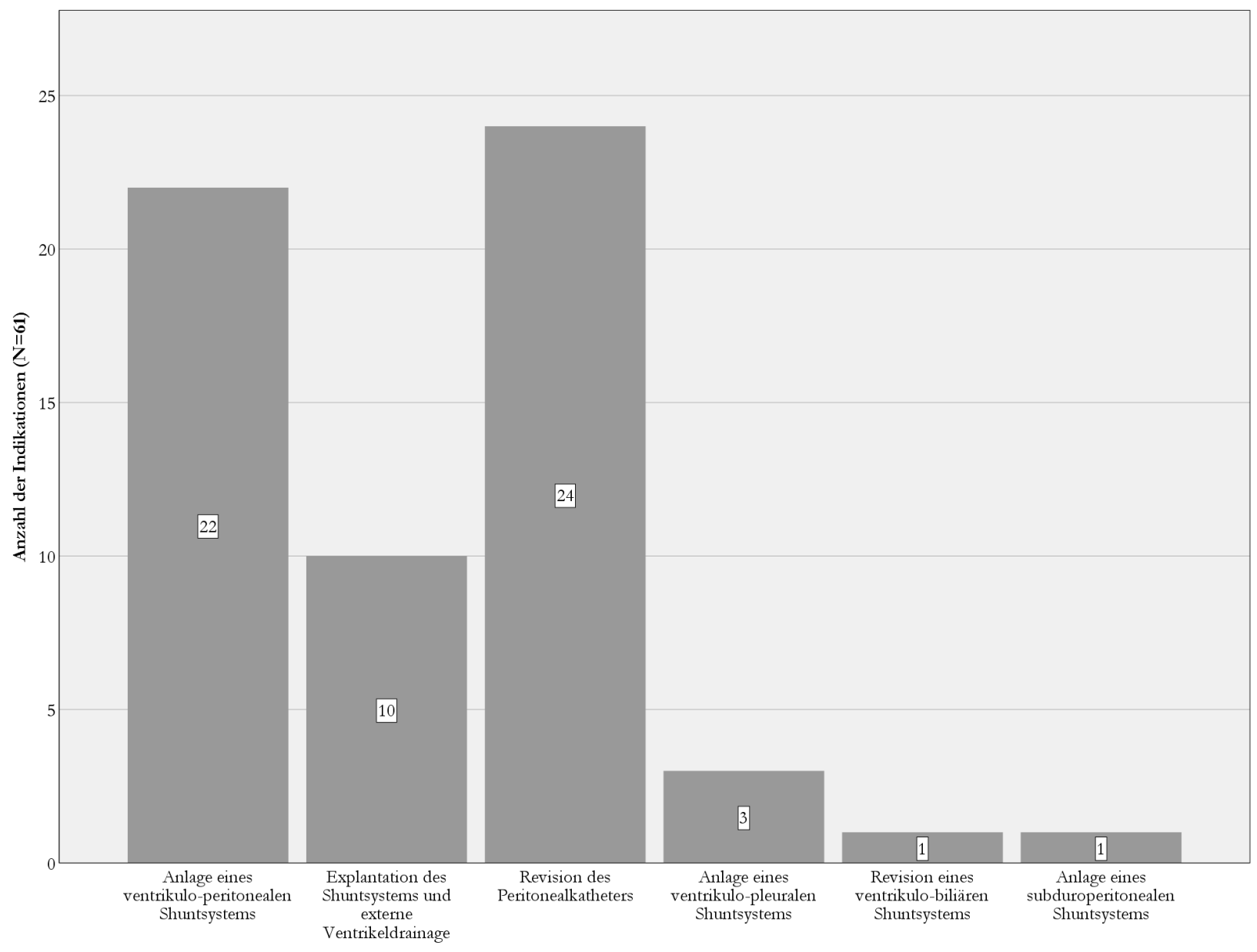

Abbildung 9: Neurochirurgische Operationsindikationen 
Die Tabelle 14 und Abbildung 9 geben einen Überblick über das Indikationsspektrum der neurochirurgischen Abteilung. Es wurde jeweils die Hauptursache der Operation vermerkt. Es gab nur eine Behandlungsindikation pro Fall. Die Hauptintention dieser Eingriffe war die Wiederherstellung eines effektiven Liquorabflusses zur Vermeidung von Folgekomplikationen. In 25 Fällen (41\%) kam es zu einer Revision des ventrikuloperitonealen Shuntsystems und in 21 Fällen $(34,4 \%)$ zu einer Neuanlage des VPS und in einem Fall zur Anlage eines subduro-peritonealen Shuntsystems nach einem Polytrauma. Damit nutzte die Mehrzahl der Fälle (77\%) nach interdisziplinärer Behandlung die abdominelle Höhle als Ort der Liquorresorption. In 10 Fällen (16,4\%) wurde eine Explantation des Shuntsystems mit externer Ventrikeldrainage durchgeführt. In drei besonderen Fällen wurde das vorhandene ventrikulo-peritoneale System in ein ventrikulopleurales System überführt. Ebenfalls erwähnenswert ist der Fall der Anlage eines ventrikulobiliären Systems. Auf diese Spezialfälle wird in der Diskussion noch einmal näher eingegangen. 


\subsubsection{Operationsindikation aus kinderchirurgischer Sicht}

Tabelle 15: Kinderchirurgische Operationsindikationen

\begin{tabular}{|c|c|c|c|}
\hline & & $\mathbf{N}$ & Prozent \\
\hline \multirow[t]{10}{*}{ Indikationen } & voroperierter Bauch & 42 & $34,4 \%$ \\
\hline & $\begin{array}{l}\text { Adhäsiolyse von Verwachsungen im } \\
\text { Bauchraum }\end{array}$ & 40 & $32,8 \%$ \\
\hline & Liquorzyste im Bauchraum & 11 & $9,0 \%$ \\
\hline & Endoskopische Bergung von Shuntmaterial & 7 & $5,7 \%$ \\
\hline & Versorgung einer Nabelhernie & 3 & $2,5 \%$ \\
\hline & Appendizitis als Komorbidität & 7 & $5,7 \%$ \\
\hline & Z. n. Peritonitis oder Abszess & 2 & $1,6 \%$ \\
\hline & Darmchirurgische Intervention & 4 & $3,3 \%$ \\
\hline & Z. n. Polytrauma & 3 & $2,5 \%$ \\
\hline & Weitere Indikationen & 3 & $2,5 \%$ \\
\hline \multicolumn{2}{|l|}{ Gesamt } & 122 & $100,0 \%$ \\
\hline
\end{tabular}

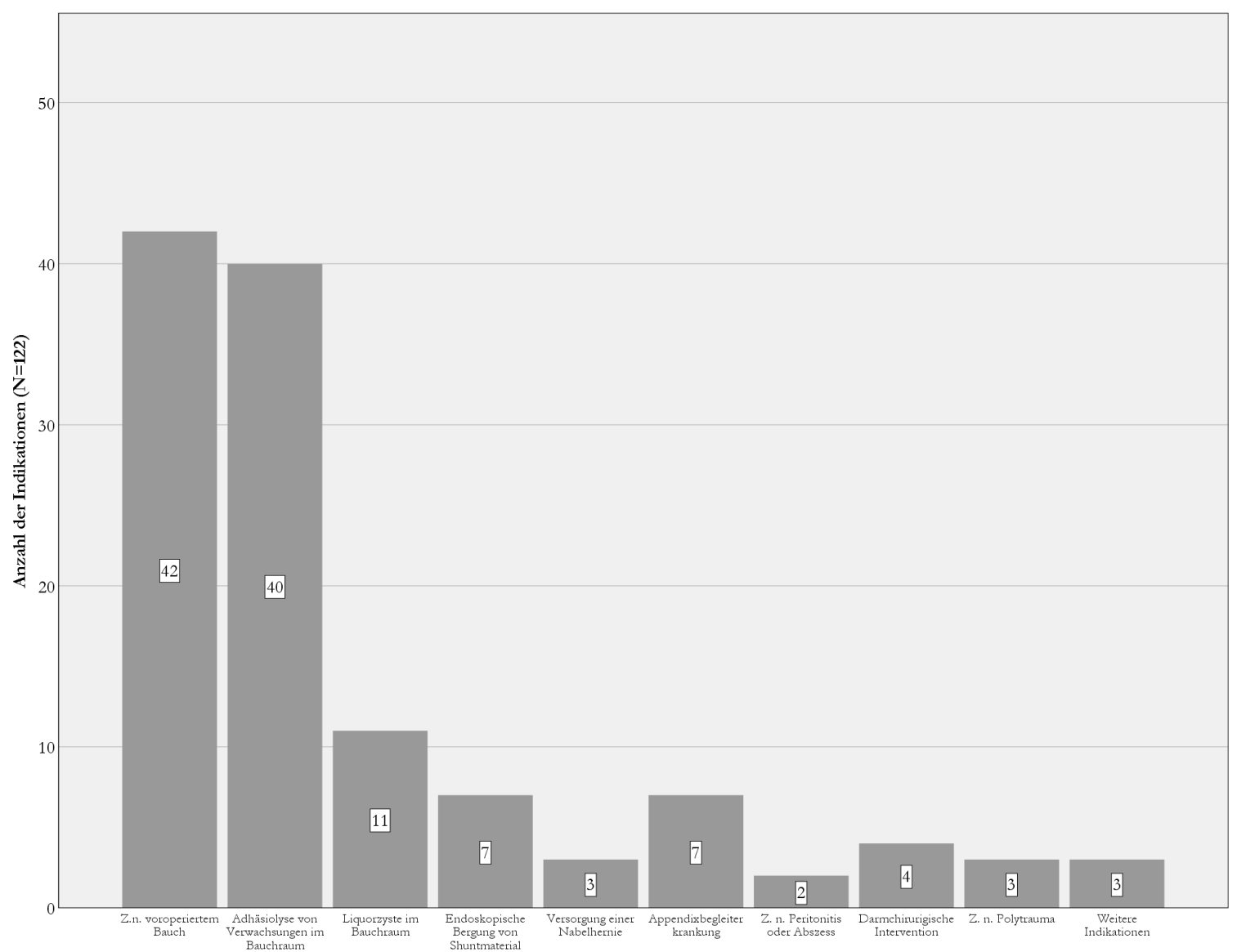

Abbildung 10: Kinderchirurgische Indikationen 
Aus der abgebildeten Tabelle 15 und Abbildung 10 gehen die kinderchirurgischen Behandlungsindikationen der interdisziplinär versorgten Fälle, die dieser Dissertation zugrunde liegen, hervor. Im Unterschied zur Neurochirurgie gab es seitens der Kinderchirurgen pro Fall oftmals mehrere Gründe für eine operative Intervention. Dies ist vor allem der Tatsache geschuldet, dass manche Komplikationen nicht isoliert voneinander betrachtet werden können. Im Diskussionsteil wird dieser Aspekt noch einmal näher erläutert. Im Durchschnitt lagen pro Fall 2 Indikationen vor. Die insgesamt 122 aufgenommenen Indikationen verteilen sich somit auf die 61 versorgten Fälle. Die am häufigsten aufgeführten Indikationen waren der „Z.n. voroperiertem Bauch $(\mathrm{N}=42)^{\text {“ }}$ und

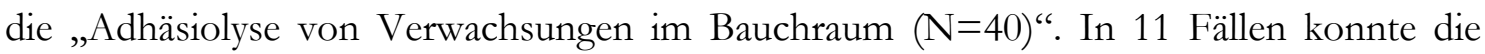
Entstehung einer Liquorzyste im Bauchraum nachgewiesen werden. Es zeigt sich, dass in 7 Fällen eine endoskopische Bergung von Shuntmaterial erforderlich war. In 3 Fällen wurde parallel zur Anlage eines VPS eine angeborene Nabelhernie mitversorgt. Im Gesamtkollektiv kam es in 7 Fällen zu einer interdisziplinären Intervention aufgrund des Vorliegens einer Wurmfortsatzentzündung bei vorhandenem VPS. In zwei Fällen trat ein Abszessgeschehen bzw. lag präoperativ eine Peritonitis vor, sodass eine gemeinsame Versorgung des Patienten notwendig war. Dreimal wurden Polytraumen in Zusammenarbeit versorgt. Ebenso dreimal war intraoperativ die Resektion von Darmanteilen aufgrund einer Kathetermigration in das Gewebe erforderlich. Die vorletzte Zeile der Tabelle mit der Rubrik „Weitere Indikation“ enthält drei interdisziplinäre Sonderfälle, in denen einmal die Herz-Thorax Chirurgen eingreifen mussten, da sich bei einem Kind eine Lungenarterienembolie entwickelt hat und in einem Fall wurde eine komplizierte Dünndarmstenose bei bestehendem Hydrozephalus operativ versorgt. Bei dem letzten Fall wurde ein Maldescensus testis mit einer Orchidopexie versorgt. 


\subsection{Analyse des Zeitraums nach interdisziplinärer Versorgung}

Tabelle 16: Deskriptive Statistik des Zeitraums nach interdisziplinärer Versorgung

\begin{tabular}{|c|c|}
\hline Weitere Intervention notwendig & 40 Fälle \\
\hline Bisher keine Intervention notwendi & 21 Fälle \\
\hline Mittelwert & 206 Tage \\
\hline Median & 35 Tage \\
\hline Standardabweichung & 373 Tage \\
\hline Spannweite & 1773 Tage \\
\hline Minimum & 3 Tage \\
\hline Maximum & 1776 Tage \\
\hline
\end{tabular}

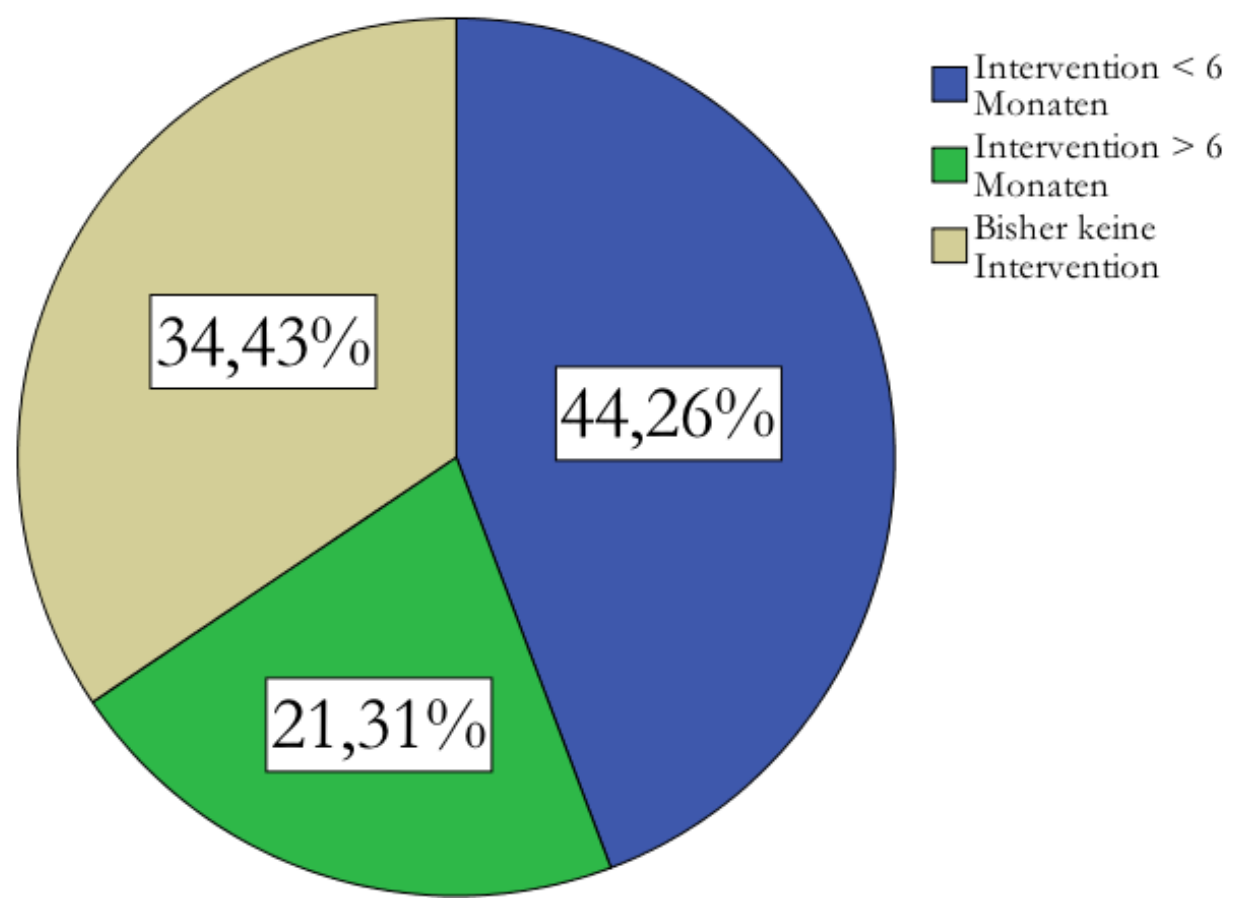

Abbildung 11: Zeitraum nach interdisziplinärer Behandlung 
Tabelle 17: Übersicht der Interventionen

\begin{tabular}{|l|c|}
\hline \multicolumn{1}{|c|}{ Intervention } & Anzahl \\
\hline Revision des Ventils & 1 \\
\hline Revision des Ventrikelkatheters & 5 \\
\hline Rekonnektion des Shuntassistenten & 2 \\
\hline Explantation des Shuntsystems & 2 \\
\hline Explantation des Shuntsystems + EVD & 3 \\
\hline EVD (Externe Ventrikeldrainage) & 2 \\
\hline Wiedereröffnung eines ligierten Systems & 1 \\
\hline Anlage eines VP-Shunts & 2 \\
\hline Externalisierung des Peritonealkatheters & 3 \\
\hline Internalisierung des Peritonealkatheters & 1 \\
\hline Revisions des Peritonealkatheters & $\mathbf{7}$ \\
\hline Umwandlung in VA-Shunt & $\mathbf{9}$ \\
\hline Revision des Subduralkatheters & 1 \\
\hline Revision einer Aquäduktstenose & 1 \\
\hline Gesamt & $\mathbf{4 0}$ \\
\hline Davon erneut interdisziplinäre Eingriffe & $\mathbf{1 1}$ \\
\hline
\end{tabular}

Die Tabelle 16 und 17 sowie Abbildung 11 geben einen Überblick über den Verlauf der Fälle nach der interdisziplinären Versorgung durch Neurochirurgie und Kinderchirurgie. Dabei wurde speziell im Kreisdiagramm eine Einteilung in Interventionen vor und nach Abschluss eines halben Jahres durchgeführt. Es wird deutlich, dass in 40 Fällen (66\%) eine weitere chirurgische Intervention innerhalb des beobachteten Zeitraums notwendig war. Dem entgegen stehen die 34\% der Fälle, die bis zum Abschluss der Datenerhebung ohne weiterführende Intervention waren. Von den durchgeführten 40 Interventionen wurden 11 $(28 \%)$ erneut interdisziplinär versorgt. Als Interventionen wurden Ereignisse definiert, die eine weitere operative Versorgung seitens der Kinderneurochirurgie, Kinderchirurgie oder beider Fachrichtungen in Zusammenarbeit notwendig machten. Dabei wurden in neun Fällen die Ableitung auf ein ventrikulo-atriales System umgestellt und in sieben Fällen würde der Peritonealkatheter revidiert. Eine Übersicht dazu gibt die Tabelle 17. Der durchschnittliche Zeitraum bis zu einer erneut notwendigen Versorgung der Kinder lag bei 206 Tagen. Die Spannweite war 1773 Tage (ca. 4,9 Jahre). Dabei kam es im frühesten Fall nach 3 Tagen zu einer erneuten Versorgung und im spätesten Fall nach 1776 Tagen. Die Standardabweichung beträgt 373 Tage. 


\section{Diskussion}

\subsection{Patienten und Methoden}

Bei der durchgeführten Datenanalyse handelt es sich um eine retrospektive Untersuchung. Die Daten wurden im Zeitraum vom 1.1.1995 bis hin zum 1.10.2018 in der Universitätsmedizin Göttingen erhoben. Das daraus entstandene Fallkollektiv von insgesamt 61 operativen Fällen beruht auf 44 Patienten. Die abgeleiteten Daten müssen aufgrund der Stichprobengröße und der weiten Individualität der Patienten zu jeder Zeit bezüglich ihrer Validität kritisch hinterfragt werden. Ähnliche Studien zu der Thematik „Abdominelle Komplikationen nach VPS-Anlage“ ließen sich zum Beispiel bei Vinchon und Dhellemmes (2005) finden. Die Mehrzahl dieser vergleichenden wissenschaftlichen Abhandlungen beinhaltet jedoch keine Fallstudien über die speziell in dieser Dissertation behandelten Fragestellungen. In diesem Zusammenhang beschäftigen sich beispielsweise die Autoren Gaskill und Marlin (1989); Aquino et al. (2006) mit einer verwandten Thematik, wobei der Faktor eventueller Voroperationen keinen Einzug in ihre Betrachtungen fand.

Ebenfalls anzumerken sind die weitgehend niedrigen Evidenzlevel in breit angelegten Reviews zur Leitlinienfindung. So werden beispielsweise die im „Journal of Neurosurgery. Pediatrics“ veröffentlichen Guidelines zum „Pediatric Hydrocephalus“ aus dem Jahre 2014 selten mit einem Evidenzniveau über Level II bewertet (Flannery und Mitchell 2014). 


\subsection{Patientencharakteristika im Vergleich}

\subsubsection{Geschlecht}

Die Zusammensetzung bezüglich des Geschlechts zwischen Mädchen (19 Patienten) und Jungen (25 Patienten) entspricht in der Tendenz anderen Studien über die Thematik des kindlichen Hydrozephalus, in denen das männliche Geschlecht ebenfalls häufiger betroffen ist (Drake et al. 2000; Tuli et al. 2000). Es ist davon auszugehen, dass es sich in der vorliegenden Dissertation um eine annährend repräsentative Stichprobe handelt.

\subsubsection{Alter zum Zeitpunkt der Versorgung}

Bei der Beobachtung des Patientenalters zum Zeitpunkt der interdisziplinären Versorgung ist ein Häufigkeitsgipfel im ersten Lebensjahr aufgefallen. Dieser erste Gipfel schließt die Versorgung der komplikationsreichen Frühgeburten ein. Laut Vinchon und Dhellemmes (2005) treten vermehrt abdominelle Probleme vor allem kurz nach der Geburt als Kombination von notwendigem Shunt und angeborener Hernie auf und dann nochmals beim Eintritt ins Jugendalter, wobei dies dem vermehrten Auftreten von Wurmfortsatzentzündungen geschuldet ist (Ohmann et al. 2002). Dieser zweite Aspekt ließ sich im Kollektiv nicht darstellen. Das Risiko einer abdominellen Komplikation ist aber zeitlebens gegeben (Vinchon und Dhellemmes 2005). 


\subsection{3 Ätiologie der Hydrozephali}

Die Verteilung der verschiedenen Hydrozephalusätiologien hat sich im Laufe der Jahre gewandelt. Der Anteil der Patienten mit Dysraphien ist durch prophylaktische Maßnahmen während der Schwangerschaft, wie zum Beispiel pränatale Diagnostik, Folsäureprophylaxe und die Möglichkeit des Schwangerschaftsabbruches zurückgegangen. Im Gegenzug dazu hat das Risiko der Entwicklung intrazerebraler Blutungen durch die erhöhte Zahl von Frühgeborenen in Folge künstlicher Befruchtung insgesamt zugenommen (Persson et al. 2007; Robinson 2012). Durch die Entwicklung der modernen Neonatologie ist die Zahl der sehr jungen Frühgeborenen vor der 28. SSW angestiegen. In der Universitätsmedizin Göttingen können Frühgeborene ab der 23. Schwangerschaftswoche versorgt werden. Bei Frühchen unter $1500 \mathrm{~g}$ beträgt das Risiko für die Entwicklung einer die germinale Matrix betreffenden Hirnblutung ca. $15 \%$ bis $20 \%$, bei Kindern unter 750 g liegt die Wahrscheinlichkeit bei bis zu 50\% (du Plessis 2009). Die dabei mögliche Folgekomplikation der Entstehung eines posthämorrhagischen Hydrozephalus mit anschließend notwendiger Shuntversorgung repräsentiert in dieser Arbeit mit 41 Fällen (67\%) die Mehrzahl der Ursachen. Andere Ursachen, wie idiopathische Hydrozephali (6 Fälle 10\%) und tumorassoziierte Hydrozephali (5 Fälle 8\%) sind am zweithäufigsten vertreten. Diese Verteilung lässt sich im Hinblick auf die Zusammensetzung der Hydrozephalusursachen in anderen Studien zum Thema kindlicher Hydrozephalus und ventrikulo-peritoneales Shuntsystem bis auf die Gemeinsamkeit des PHHC als Risikofaktor für Komplikation nicht bestätigen (Serlo et al. 1990; Tuli et al. 2000). Vor diesem Hintergrund untersucht die Studie von Tuli et al. (2000) die Ursachen für ein wiederholtes Shuntversagen bei Kindern und damit verbundene Risikofaktoren.Die Ergebnisse zeigen, dass neben dem Alter der initialen Shuntversorgung auch Ursachen wie PHHC, HC n. entzündlicher Genese und Neoplasien (tumor-assoziierte HC) ein erhöhtes Risiko für ein Shuntversagen bedingen. Ergebnisse der Studie „Komplikationen von VPS bei Kindern bezogen auf verschiedenen Ätiologien“ von Serlo et al. (1990) ergänzen diesen Sachverhalt durch die Aussage, dass tumor-assoziierte HC eine höhere Infektionsrate nach sich ziehen, da der Liquor durch den erhöhten Anteil von Proteinen vermutlich eine verstärkte immunologische Antwort indiziert. Die Ursache des Hydrozephalus, welcher zur Shuntanlage geführt hat, spielt jedoch laut Vinchon und Dhellemmes (2005) für das Auftreten der speziellen abdominellen Komplikation nur bedingt eine Rolle. Sie konnten nachweisen, dass alle Hydrozephalusätiologien abdominelle Komplikationen nach sich ziehen können, aber Patienten mit tumor-assoziiertem HC eher weniger und Patienten mit postinfektiösem HC und kommunizierendem $\mathrm{HC}$ eher mehr betroffen waren. Aus diesem Grund muss der in Tabelle 18 exemplarisch durchgeführte 
Vergleich der Ätiologieverteilung sehr kritisch hinterfragt werden, wobei Sie dennoch einen hilfreichen Überblick über die sehr variablen Verteilungsformen gibt.

Tabelle 18: Vergleich der Ätiologieverteilung verschiedener Studien

\begin{tabular}{|l|c|c|c|}
\hline \multicolumn{1}{|c|}{ Ursache } & Eigene N=61 & $\begin{array}{c}\text { Serlo et al. (1990) } \\
\mathbf{N = 3 0 6}\end{array}$ & $\begin{array}{c}\text { Tuli et al. (2000) } \\
\mathbf{N = 8 9 3}\end{array}$ \\
\hline $\begin{array}{l}\text { Posthämorrhagischer HD bei } \\
\text { Frühgeburtlichkeit }\end{array}$ & $69 \%$ & $22,5 \%$ & $13,6 \%$ \\
\hline Posttraumatischer HD & $5 \%$ & Keine Angabe & $2,3 \%$ \\
\hline Dysraphien & $7 \%$ & $13,1 \%$ & $22,8 \%$ \\
\hline Tumor-bedingter HD & $8 \%$ & $4,2 \%$ & $22,6 \%$ \\
\hline $\begin{array}{l}\text { HD bei sonstiger ZNS } \\
\text { Fehlbildung }\end{array}$ & $3 \%$ & $37,3 \%$ & $17,5 \%$ \\
\hline $\begin{array}{l}\text { HD entzündlich oder } \\
\text { idiopathischer Genese }\end{array}$ & $8 \%$ & $22,9 \%$ & $21,2 \%$ \\
\hline
\end{tabular}

Eine alternative Einteilung der Ätiologie bzw. eine noch detailliertere Untersuchung der in Kapitel 1.3 aufgeführten Ursachen wurde aufgrund der fehlenden Relevanz für die nicht primär ätiologiebedingte Fragestellung dieser Arbeit nicht vorgenommen. 


\subsubsection{Anzahl der Shuntrevisionen}

Im Durchschnitt waren bei jedem Patienten 3,2 Revisionsoperationen notwendig. Dies schließt Shuntrevisionen aufgrund von Komplikationen oder Wachstum mit ein. Durchschnittswerte anderer Studien sind in Tabelle 19 aufgeführt.

Tabelle 19: Shuntrevisionen im Vergleich

\begin{tabular}{|l|c|c|c|c|}
\hline \multicolumn{1}{|c|}{ Studie } & Patienten & Mittelwert & Revisionsoperationen & Ätiologie \\
\hline Eigene & 44 & 3,3 & 144 & Gemischt \\
\hline Chittiboina et al. (2013) & 78 & 3,9 & 304 & Nur PHHC \\
\hline Bir et al. (2016) & 122 & 0,9 & 108 & Nur PHHC \\
\hline Pan (2018) & 134 & 0,4 & 54 & Gemischt \\
\hline Månsson et al. (2017) & 434 & 0,4 & 185 & Gemischt \\
\hline
\end{tabular}

Die hohe Revisionsrate im Gegensatz zu anderen Studien unterstreicht nochmals, wie hochmorbide und komplex das dieser Dissertation zugrunde liegende Patientenkollektiv ist. Nur Bir et al (2016) haben eine vergleichbare Anzahl an Revisionsoperationen registriert. Die anderen Studien liegen weit darunter. Eine interdisziplinäre Versorgung des hier vorliegenden Patientenklientels ist somit die logische Konsequenz zur ganzheitlichen Behandlung der Kinder. 


\subsection{Risikofaktoren für abdominelle Shuntkomplikationen}

\subsubsection{Posthämorrhagischer Hydrozephalus in Bezug zum Blutungsgrad nach Papile}

Bei den im Kollektiv untersuchten 41 Fällen von PHHC mit intraventrikulärer Hirnblutung war in allen Fällen der Faktor Frühgeburtlichkeit erfüllt. Die hohe Anzahl der höhergradigen Blutungen lässt darauf schließen, dass die Schwere der Blutung mit dem Auftreten des PHHC verbunden ist. Laut Volpe (1995) ist die Wahrscheinlichkeit, einen PHHC zu entwickeln, bei einer Grad-III-Blutung 55\% und bei einer Grad-IV-Blutung 80\%. Ähnlich äußern sich Bir et al. (2016). Weiterhin scheint der Score von Papile einen prognostischen Wert in Bezug auf Mortalität und neurologisches Outcome zu haben (Papile et al. 1980; Pinto-Martin und Riolo 1995).

Chittiboina et al. (2013) haben als Grundlage ein Patientenkollektiv, welches nur aus Patienten mit einem PHHC besteht. Sie beschreiben eine erhöhte Anfälligkeit für Shuntrevisionen im Vergleich zu anderen Studien. Ebenso lässt sich auch durch den hohen Anteil der Fälle in dem hier vorliegenden untersuchten Kollektiv mit PHHC als Hydrozephalusätiologie vermuten, dass diese Ursache einen Risikofaktor für die Entwicklung von abdominellen Komplikationen bei stattgehabter Anlage eines ventrikuloperitonealen Shuntsystems darstellt.

Darüber hinaus sollte die interdisziplinäre Zusammenarbeit, die bei der Versorgung des PHHCs notwendig ist, auch nach der definitiven Versorgung mit einem Shuntsystem fortgeführt werden und jederzeit an die Möglichkeit der Entwicklung von abdominellen Komplikationen gedacht werden.

Die Zukunft in der Behandlung des PHHC liegt wohl in der weiteren Verbesserung der neonatologischen Versorgung in Kombination mit standardisierter chirurgischer Therapie. Die Berliner Arbeitsgruppe um M. Schulz und U.-W. Thomale folgt seit 2010 erfolgreich einem neuen Behandlungskonzept. Die Blutungen werden mithilfe einer endoskopischen Lavage durch 37 Grad warme laktatfreie, isotonische Natriumchloridösung behandelt, um somit eine spätere Malabsorption des Liquors zu verhindern (Schulz et al. 2014). 


\subsubsection{Frühgeburtlichkeit als Risikofaktor für abdominelle Komplikationen nach ventrikulo-peritonealer Shuntversorgung}

In 48 von 61 Fällen im Fallkollektiv konnte eine Frühgeburtlichkeit nachgewiesen werden. Diese Tatsache unterstreicht die in der Literatur beschriebene Korrelation zwischen einer erhöhten Hydrozephaluswahrscheinlichkeit und Geburt vor der 37. Schwangerschaftswoche.

Der Zusammenhang zwischen Frühgeburtlichkeit und erhöhter Komplikationsanfälligkeit der Shuntversorgung konnte auch im Gesamtkollektiv der erhobenen Daten aus dem Göttinger Shuntregister herausgestellt werden. Dabei war das komplikationsfreie Intervall nach VPS-Anlage bei Reifgeborenen signifikant höher als bei Frühgeborenen (Bock et al. 2015). Die mediane Shuntüberlebensrate bei Frühgeborenen lag bei 60,5 Monaten gegenüber 124,6 Monaten bei über der 37. SSW geborenen Kindern.

Vor dem Hintergrund der herausgestellten Daten ist hierbei nicht nur die Frage des Zeitpunkts zur Versorgung des kindlichen Hydrozephalus relevant, sondern auch mögliche Behandlungsoptionen. Wie schon erwähnt, ist in diesem Zusammenhang zu klären, ob eine möglichst frühe Shuntversorgung insbesondere bei Frühgeborenen durchgeführt werden sollte oder ob dem Hirndruckanstieg mittels temporärer Lösungen, wie durch das Anlegen einer RICKHAM-Kapsel oder durch wiederholte Lumbalpunktionen entgegenzuwirken erfolgreicher ist (Bock et al. 2018a). Die Meinungen darüber gehen weit auseinander und werden kontrovers diskutiert (Riva-Cambrin et al. 2012). Die Early Versus Late VentricularIntervention Study (ELVIS) untersucht diesen Faktor in einer breit angelegten Studie (Kahle et al. 2016).

Die Fallanzahl des hier interdisziplinär untersuchten Kollektivs, welche den Faktor Frühgeburt erfüllt haben, deuten darauf hin, dass die Frühgeburtlichkeit nicht nur Risikofaktor für generelle Shuntkomplikationen ist, sondern in diesem Zusammenhang auch verstärkt abdominelle Komplikationen nach VPS-Anlage in Betracht gezogen werden müssen. 


\subsubsection{Risikofaktor Geburtsgewicht}

Bei der Analyse des Geburtsgewichts der Frühchen wurden im Durchschnitt 1033 g ermittelt. Der Durchschnittswert von Reifgeborenen der 37. SSW liegt bei ca. 2900 g (Fenton und Kim 2013). Niedriges Geburtsgewicht ist ein Risikofaktor für Folgekomplikationen nach Shuntoperationen (Chittiboina et al. 2013; Bir et al. 2016). Dabei geben Studien einen deutlichen Risikoanstieg bei einem Gewicht unter $1500 \mathrm{~g}$ an. (Gurtner et al. 1992; Bir et al. 2016). An der Universitätsmedizin Göttingen liegt die interne Grenze für Shuntoperationen bei 2500 g. Tritt bei Kindern unter dieser Grenze ein Hydrozephalus auf, dann werden diese mit einem temporären subgalealem Reservoir zur Liquorpunktion versorgt, bis die angestrebte Grenze erreicht ist (Bock et al. 2018). Ziel ist die Reduktion von Folgekomplikationen und das Verhindern von frühen Shuntrevisionen (Willis et al. 2009). Die Wahrscheinlichkeit, dass ein geringes Geburtsgewicht ein erhöhtes Risiko für abdominelle Komplikationen nach VPS-Anlage mit sich bringt, ist sehr hoch. Aufgrund des hier vorliegenden kleinen Fallkollektivs ist diese Aussage jedoch nur gering valide.

\subsubsection{Nekrotisierende Enterokolitis als Risikofaktor für abdominelle Kompli- kationen nach ventrikulo-peritonealer Shuntversorgung}

In den 48 Fällen mit Frühgeburtlichkeit wiesen 23 Fälle (38\%) die Komorbidität der nekrotisierenden Enterokolitis auf. In der Literatur sind bezüglich der NEC als Komorbidität unterschiedliche Inzidenzzahlen zu finden. Mobley et al. (2005) zeigten in ihrer Studie über abdominelle Liquorpseudozysten bei liegendem VPS, dass 19\% der Patienten eine NEC in der Anamnese vorwiesen. Spader et al. (2015) registrierten in ihrem Kollektiv bei 11,4\% der Patienten eine nekrotisierende Enterokolitis als Komorbidität. Die in unser Untersuchung ermittelten Werte liegen mit 38\% deutlich darüber. Dies zieht die Frage nach sich, ob diese Frühgeborenenkomplikation einen generellen Risikofaktor für abdominelle Probleme bei shuntversorgtem Hydrozephalus darstellt. Diese These kann anhand der eigenen Untersuchungen bestätigt werden. Die pathophysiologischen Prozesse der NEC können Verwachsungen und Vernarbungen begünstigen und im Falle eines VPS-versorgten Hydrozephalus notwendige peritoneale Liquorresorption kompromittieren. Dies trifft auch auf weitere abdominelle Operationen, wie beispielsweise nach Mekoniumileus oder Volvulus zu (Kargl et al. 2017). In der Literatur finden sich diesbezüglich aber nur wenig vergleichende Studien. 


\subsubsection{Infektionsnachweis bei abdominellen Komplikationen}

In dem der Arbeit zugrunde liegenden Fallkollektiv konnten in 10 Fällen (16\%) Infektionen mit oder ohne Keimnachweis festgestellt werden. Dabei wurden 6 verschiedene Bakterien detektiert. In 4 Fällen war keine Sicherung des Erregers möglich. Die vorliegenden Ergebnisse bewegen sich somit innerhalb der in der Literatur beschriebenen Spannweite der Infektionsraten nach shuntversorgtem HC von 3\% bis 27,6\% (Prusseit et al. 2009). Eine superinfizierte abdominelle Liquorpseudozyste als seltene Ausprägung der Infektion konnte in einem Fall im Kollektiv beobachtet werden. Das dabei ursächliche Bakterium war nicht nachweisbar. Eine allgemeingültige Aussage über den Sachverhalt von Infektionen als Risikofaktor für die Entwicklung abdomineller Komplikationen bei liegendem VPS kann auf der Grundlage der hier erhobenen Daten nicht getroffen werden. Dennoch sei an dieser Stelle nochmals die Infektion des Shuntsystems als entscheidende Hauptkomplikation bei der Hydrozephalusversorgung dargestellt, welche in der Behandlung der Kinder jederzeit bedacht werden muss (Tamber et al. 2014). 


\subsection{Interdisziplinäre Versorgung abdomineller Shuntkomplikationen}

\subsubsection{Operationszeitpunkt, Operationszugänge und Ableitungsart nach chirurg- ischer Versorgung des distalen Shuntkatheters}

Im Kapitel 3.3.3 wurde die Dringlichkeit der interdisziplinär durchgeführten Operationen untersucht. Entgegen den Erwartungen stellte sich heraus, dass die Mehrzahl der gemeinsamen Operationen (70,5\%) elektiv bzw. mit zeitnaher Terminierung durchgeführt wurde. Nur 18 Fälle $(29,5 \%)$ wurden notfallmäßig operiert. Dabei ist jedoch zu berücksichtigen, dass Notfälle in Verbindung mit einem kindlichen Hydrozephalus immer mit einem hohen Risikoprofil für den Patienten behaftet sind, wenn die Operation nicht sehr rasch durchführt werden. Dabei muss zwischen einer lebensbedrohlichen Situation aufgrund eines erhöhten Hirndrucks durch Shuntdysfunktion und den potentiellen Gefahren einer Infektion des ZNS unterschieden werden. Innerhalb der elektiven Fälle erfolgte dreimal eine interdisziplinäre Mitbehandlung durch den Kinderchirurgen, da eine angeborene Nabelhernie versorgt werden musste. Narkosen bei Kindern können mutmaßlich mit erhöhtem Risiko für neurologische Folgeschäden behaftet sein, zumindest wird dies in der Literatur diskutiert (Davidson et al. 2016). In Anbetracht dieser Aussagen und auch aus Effektivitätsgründen erfolgten die Eingriffe zur Shuntversorgung des Hydrozephalus und die Hernienversorgung in einer Operation. In weiteren elektiven Fällen handelt es sich um Kinder, die vorübergehend mit einer RICKHAM-Kapsel oder einem externalisierten Shuntsystem zur Liquordrainage versorgt waren und deren neue permanente Versorgung durch beispielsweise ein ventrikulo-peritoneales Shuntsystem erst nach dem Abklingen der Infektparameter möglich war, wie von Vinchon und Dhellemmes (2005) empfohlen. Schlussfolgernd lässt sich aus den vorliegenden Ergebnissen vermuten, dass die interdisziplinäre Versorgung von Kindern mit abdominellen Shuntproblemen planbarer ist als erwartet. Es wird in diesem Zusammenhang jedoch als besonders wichtig erachtet, dass eine einmal begonnene interdisziplinäre Versorgung insbesondere bei notfallmäßigen Fällen fortgeführt wird, um ein optimales Komplikationsmanagement zu gewährleisten und somit das Outcome für diese Patienten zu verbessern. 
Studien, welche das Outcome von Patienten mit Shuntversorgung bei unterschiedlichen Operationstechniken untersuchen, sind in diesem Zusammenhang kritisch zu betrachten, da sie sich in den meisten Fällen auf die Untersuchung von Erwachsenen begrenzen und dadurch nicht mit dem hier vorliegenden, sehr selektiertem Kollektiv verglichen werden können.

Beim vorliegenden Patientenkollektiv kamen sowohl offene als auch endoskopische Operationsverfahren zum Einsatz. In der Mehrzahl (77\%) wurden die Fälle durch offene chirurgische Verfahren versorgt. Dies beinhaltet die klassische offene Laparotomie und die Minilaparotomie. Das offene Verfahren zeichnet sich insbesondere durch eine gute Beherrschbarkeit von intraoperativen Komplikationen aus (Lifshutz und Johnson 2001; Phan et al. 2016). In 10 Fällen wurde eine Minilaparotomie durchgeführt. Die Misserfolgsrate dieses Verfahrens liegt je nach herangezogener Studie zwischen 6\% und 29\%. Dem entgegen steht die Komplikationsrate der klassischen laparoskopischen Insertionen von 0\% - 16\% (He et al. 2016). In dem vorliegenden Patientenkollektiv konnten alle Operationen erfolgreich durchgeführt werden.

Ebenso wurden 10 Fälle durch minimalinvasive endoskopische Zugänge zur Bauchhöhle versorgt. Insbesondere bei Katheterdiskonnektionen hat sich dieses Operationsverfahren im hier untersuchten Kollektiv bewährt, da eine Bergung unter direkter Sicht erfolgen konnte. Weiterhin ist das Risiko für Darmverletzungen, postoperative Hernien, Gesamtschmerzen und postoperative Verwachsungen minimiert. Gleichzeitig kann das Einbringen eines neuen distalen ventrikulo-peritonealen Shuntsystems unter direkter Sicht erfolgen (He et al. 2016; Phan et al. 2016).

Ein allgemeiner Konsens über die beste Methode der Shuntinsertion des distalen VPS herrscht in der Literatur bis heute nicht vor (Nigim et al. 2014; He et al. 2016; Phan et al. 2016). Die Grenzen der endoskopischen Versorgung sind trotz der Entwicklung spezieller Operationsinstrumente bei den kleinen Bauchhöhlen der Kinder, insbesondere der Frühgeborenen, erreicht. Gerade die beispielsweise nach NEC gespannte Bauchdecke kann durch die Insufflation des Gases bei endoskopischen Operationen weiter geschädigt werden (Burgmeier und Schier 2016). Auch der kosmetische Aspekt einer minimalinvasiven Vorgehensweise bei Kindern rückt aufgrund des noch ausstehenden Wachstums und der Narbenveränderungen immer weiter in den Vordergrund (Phan et al. 2016). Weiterhin waren die Hauptoperationsindikationen seitens der Kinderchirurgen in den hier zugrunde liegenden Fällen das Vorliegen von Verwachsungen durch Voroperation und der Zustand nach nekrotisierender Enterokolitis. Aus diesem Grund wurde in der Mehrzahl der Fälle der 
standardisierte offene Zugang über eine Laparotomie gewählt. Dennoch sprechen sich Carvalho et al. (2014) und Johnson und Pimpalwar (2009) für den therapeutischen Nutzen endoskopischer Verfahren gerade nach stattgehabten intraabdominellen Problemen bzw. multiplen Revisionen nach VPS-Anlage aus.

Im vorliegenden Fallkollektiv behielten 76\% der Fälle (46) auch im weiteren Verlauf eine ventrikulo-peritoneale Ableitung. Zehn Fälle (16\%) wurden externalisiert. Die restlichen 5 Sonderfälle $(8 \%)$ werden im anschließenden Text nochmals näher erläutert.

Die Bauchhöhle bleibt auch bei Patienten mit Begleitkomplikationen und komplexen Komorbiditäten, die eine interdisziplinäre Versorgung notwendig machen, der Ort der Wahl für eine Shuntversorgung. Ähnlich verhalten sich die Kollektive von Bir et al. (2016) und Chittiboina et al. (2013), in denen die VPS auch nach multiplen Revisionen bestehen bleiben. Trotzdem sollten vor jeder Operation alle Möglichkeiten in Betracht gezogen werden und die weitere Notwendigkeit des Shunts hinterfragt werden. Eventuell profitiert der Patienten von einem anderen Behandlungskonzept des Hydrozephalus, wie beispielsweise einer ETV bzw. bietet eine Shuntumwandlung bessere Chancen zur suffizienten Liquordrainage (Vinchon und Dhellemmes 2005; Morosanu et al. 2018). 


\subsubsection{Kinderchirurgische abdominelle Komplikationen}

In $67 \%$ der interdisziplinär versorgten Fälle wurden die Behandlungsindikationen voroperierter Bauchhöhle und Adhäsiolyse registriert. Es musste eine Adhäsiolyse durchgeführt werden, um eine erneute freie Liquorresorption in der gesamten peritonealen Höhle zu gewährleisten. Grant et al. (2008) haben dies in ihren Untersuchungen in vergleichbarem Umfang festgestellt. Das Risiko für postoperative Adhäsion im Bauchraum ist von der Art des Eingriffs und der operierten Seite abhängig. Dieses konnte im vorliegenden Patientenkollektiv nicht bestätigt werden.

Im Fallkollektiv wurden in 7 Fällen Patienten aufgrund einer Appendizitis im zeitlichen Zusammenhang mit der interdisziplinären Shuntrevision versorgt. Dies beinhaltet Kinder mit akuter Infektion und Kinder im postoperativen Fenster nach stattgehabter Appendektomie und gleichzeitig vorliegendem VPS. Das Krankheitsbild ist im Allgemeinen ohne Konsequenzen für Kinder mit einem shuntversorgten Hydrozephalus. Probleme entstehen erst, wenn es infolge einer Perforation oder Translokation von Mikroorganismen zu einem entzündlichen Geschehen im Bauchraum kommt. Dieses wurde in zwei Fällen registriert. Es lag eine Begleitinfektion vor und in einem Fall gelang der Nachweis von Staphylococcus aureus als ursächliches Bakterium. Die resultierende Peritonitis mit möglicher Sepsisfolge ist ebenso lebensbedrohlich, wie Infektion und Kontamination des Shuntsystems (Vinchon und Dhellemmes 2005). In den meisten Fällen ist der mikrobiologische Liquorbefund bei Shuntinfektion durch Appendizitis-induzierte Peritonitis negativ, sodass auf ein hirnschrankengängiges Antibiotikum verzichtet werden kann (Rekate et al. 1979; Hadani et al. 1982; Pumberger et al. 1998). Verkomplizierend kommt bei Kindern mit VPS die Möglichkeit einer Shuntfehlfunktion als Ursache für die Krankheitssymptome hinzu, welche in manchen Fällen dann zu einer verzögerten Operationsindikation mit steigendem Perforationsrisiko führt (Pumberger et al. 1998; Barina et al. 2007). Auch diagnostische Befunde, wie beispielsweise ein entzündlicher Vorgang um die intraperitoneal liegende Katheterspitze, können bei einer akuten Appendizitis ähnlich sein und die richtige Diagnosestellung dadurch erschweren (Leibrock et al. 1976).

Im vorliegenden Fallkollektiv konnte bei insgesamt 11 Fällen eine Liquorpseudozyste nachgewiesen werden. Dies entspricht 9\% aller Fälle. Exemplarisch ist in Abbildung 13 die laparoskopische Sicht auf das intraoperative Feld bei einem dieser Fälle dargestellt. In drei Fällen lag eine superinfizierte Zyste vor, bei der allerdings kein Keimnachweis erbracht werden konnte. Sieben weitere Fälle wiesen keine nachweisbare Infektion auf und in einem 
Fall konnte keine Angabe über den Infektionsgrad gemacht werden. Die Infektionsraten schwanken laut Literatur zwischen 17\% und 80\% mit einem Durchschnitt von 41\% (Siehe dazu Tabelle 20). Die in allen Studien am häufigsten isolierten Keime waren Staphylococcus aureus und Staphylococcus epidermidis. Das vorliegende Patientenkollektiv liegt innerhalb der in der Literatur beschrieben Werte.

Tabelle 20: Infektionsraten von abdominellen Liquorzysten in verschiedenen Fallstudien

\begin{tabular}{|l|c|c|c|}
\hline \multicolumn{1}{|c|}{ Quelle } & & & \\
& Anzahl Liquorzysten & Davon Infiziert & Infektionsrate \% \\
\hline Eigene & 11 & 3 & 27 \\
\hline Egelhoff et al. & 10 & 8 & 80 \\
\hline Ersahin et al. & 10 & 6 & 60 \\
\hline Gutierrez und Raimondi & 11 & 5 & 45 \\
\hline Gaskill et. al. & 12 & 2 & 17 \\
\hline Rainov et al. & 14 & 4 & 29 \\
\hline Salomao und Leibinger & 18 & 8 & 44 \\
\hline Hahn et al. & 26 & 9 & 36 \\
\hline Roitberg et al. & 27 & 12 & $\mathbf{4 1}$ \\
\hline Gesamt & $\mathbf{1 2 9}$ & $\mathbf{5 7}$ & \\
\hline
\end{tabular}

Quelle: (Mobley et al. 2005) S.80 Tabelle 5

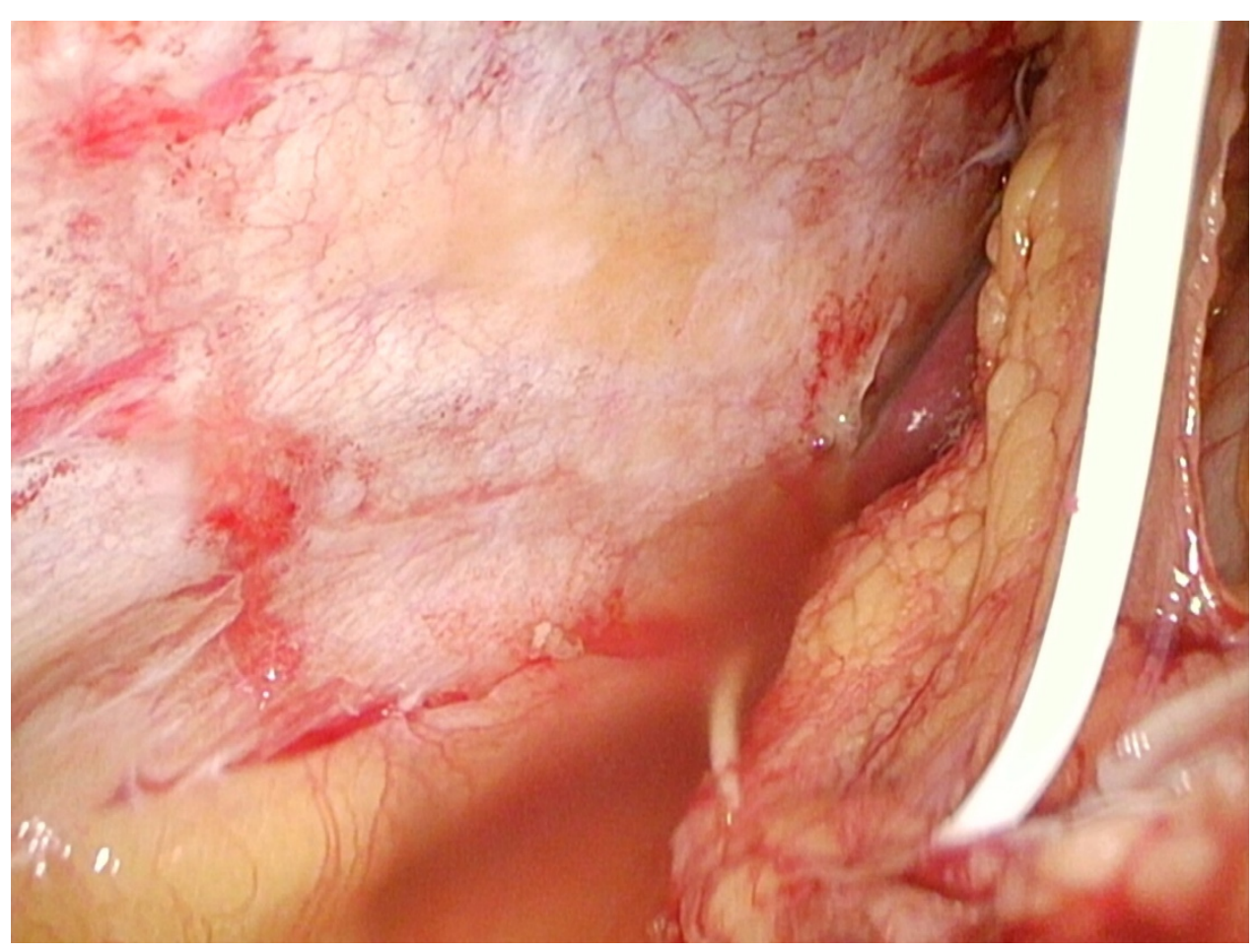

Abbildung 12: Ventrikulo-peritonealer Shunt in nicht infizierter Liquorpseudozyste 
Eine Differentialdiagnose zur oben genannten Pseudozyste ist die Aszites, welche sich durch ähnliche klinische Symptome äußert, aber im Gegensatz zur Zyste das gesamte Abdomen betrifft. Die Anzahl der diesbezüglich in der Literatur dokumentierten Fälle ist sehr gering. In dem hier untersuchten Kollektiv konnte kein Fall beobachtet werden. Rush et al. (1985) geben in ihrer Patientenserie 1,3\% als Inzidenz an (4 von 300 langzeituntersuchten Patienten). Gleichwohl ist eine Entstehung von Aszites infolge einer peritonealen Shuntanlage möglich.

Angeborene indirekte Leistenhernien und Hydrozelen bei Jungen bzw. Nuck'sche Zysten bei Mädchen sind häufige Operationsindikationen bei Kindern und Jugendlichen. Insbesondere das männliche Geschlecht ist davon vermehrt betroffen und auch in dem hier untersuchten Kollektiv konnten drei Fälle von Nabelhernien bei Jungen nachgewiesen werden. Clarnette et al. (1998) geben an, dass 15\% aller Kinder mit VPS eine Hernie entwickeln. Insbesondere Kinder unter fünf Jahren und in einem Zeitraum von zwei Jahren nach Shuntanlage sind besonders gefährdet (Chen et al. 2011). Als mögliche Gründe dafür werden unter anderem erhöhte intraabdominelle Drücke durch den Liquorabfluss angegeben (Çelik et al. 2005).

Eine notwendige endoskopische Bergung von Shuntmaterial seitens der Kinderchirurgen war in 6 Fällen notwendig. An dieser Stelle wird auf das Kapitel 1.8 verwiesen, in dem das mechanische Versagen von ventrikulo-peritonealen Shuntsystemen im Detail erklärt ist.

In zwei Fällen des Kollektivs kam es zu einer Darmperforation und Kathetermigration durch den liegenden Shunt. Die Inzidenzraten dafür sind sehr niedrig und werden in der Literatur mit 0,01\% - 0,07\% angegeben. Die ersten beiden Fälle beschrieben Wilson und Bertan (1966). Pathophysiologisch ist nicht genau klar, wie es zur Hohlorganperforation kommt. Wahrscheinlich sind Faktoren wie chronische Irritationen, allergische Reaktionen zum Beispiel durch Silikon oder scharfe Kanten der Shuntspitze verantwortlich. Ebenso sind Vorerkrankungen, Voroperationen und der medizinische Allgemeinzustand ausschlaggebend (Sharifian et al. 2013; Burhan et al. 2018). Therapeutisch ist eine operative Exploration mit eventueller Darmteilresektion sowie Reanastomose erforderlich. Auch eine Explantation des Shuntsystems sowie eine antibiotische Abschirmung sind sinnvoll (Burhan et al. 2018). Die Patienten im vorliegenden Kollektiv wurden auf diese Art und Weise versorgt. 


\subsubsection{Sonderfall Polytrauma}

Im Kollektiv wurden drei Fälle der interdisziplinären Versorgung von Neurochirurgie und Kinderchirurgie erfasst, bei denen Patienten infolge eines Polytraumas durch einen Verkehrsunfall versorgt wurden. In einem Fall erfolgte von neurochirurgischer Seite zuerst die Anlage eines subduroperitonealen Shunts, welcher zwei Monate später bei ausbleibender Normalisierung des ICPs in ein ventrikulo-peritoneales Shuntsystem konvertiert wurde. Die anderen beiden Fälle waren interdisziplinäre Revisionsoperationen bei liegendem Shuntsystem. Die Kinderchirurgen begleiteten diese Operationen durch explorative Laparotomien und Versorgung eines abdominellen Kompartmentsyndroms. Die Versorgung von Polytraumen stellt eine wichtige Schnittstelle zwischen allen Fachdisziplinen der Medizin dar.

\subsubsection{Neurochirurgische Operationsindikationen der Fälle}

Die Operationsindikationen aus neurochirurgischer Sicht beziehen sich vorrangig auf Neuanlage von VPS (21 Fälle 34,4\%) und Revisionsoperationen bei vorhandenem VPS (25 Fälle 41\%). Dabei zählen zu den Neuanlagen auch Patienten, die nach vorrübergehender externer Ventrikeldrainage zum Beispiel nach stattgehabter manifester Shuntinfektion wieder erneut mit VPS versorgt werden.

Trotz allen wissenschaftlichen Fortschritts werden beispielsweise in Nordamerika jährlich bis zu 18.000 Shuntoperationen durchgeführt und damit Kosten von etwa 1 Milliarde US\$ generiert. Hinzu kommen etwa 20.000 weitere stationäre Patientenfälle bei denen Kinder mit VPS versorgtem Hydrozephalus aufgrund von Revisionen oder Komplikationen behandelt werden müssen (Simon et al. 2008). Dies spiegelt in etwa die in dem untersuchten Kollektiv gefundene Verteilung von Revisionen und Neuanlagen wieder. Wie bereits eingangs erwähnt, ist die Misserfolgsrate für alle implantierten Shuntsysteme hoch und liegt bei ca. $40 \% \mathrm{im}$ ersten Jahr und ca. 50\% im Verlauf von zwei Jahren (Baird et al. 2014). Je nach Art der Komplikation kann auch eine zeitweise Externalisierung des Shunts bzw. eine Entfernung von Teilkomponenten oder des gesamten Systems notwendig sein. Gründe hierfür sind zum Beispiel floride, nicht mit Antibiotika behandelbare Infektionen oder abdominelle Malabsorption des Liquors (Tamber et al. 2014). Dies wurde hier in insgesamt neun Fällen (20\%) beobachtet. 
Trotz der Vielzahl der Komplikationen ist die Mortalitätsrate bei Shuntoperationen verhältnismäßig gering und liegt perioperativ bei ca. 0,5\% und sinkt, wenn die Operation in einem Krankenhaus der Maximalversorgung erfolgt (Smith et al. 2004). Durchschnittlich geben Vinchon et al. (2012) eine 30-Jahre-Mortalität zwischen 5\% und 10\% an.

Neben den oben genannten, eher klassischen neurochirurgischen Operationsindikationen, wurden auch zwei sehr spezielle Liquorableitungen im interdisziplinären Fallkollektiv versorgt.

Der erste Fall betraf einen Patienten mit einem ventrikulo-cholezystischem Shuntsystem, welches den Liquor aus dem Ventrikelsystem in die Gallenblase drainiert. Aufgrund von postoperativen persistierenden Bauchschmerzen wurde der Patient in Gemeinschaft mit den Kinderchirurgen versorgt. Intraoperativ zeigten sich Adhäsionen und Verwachsungen des Shunts am Übergang von extra- nach intraperitoneal. Nach Lösung dieser Fixierungen waren die Schmerzen des Patienten im weiteren Verlauf rückläufig. Bei der verwendeten Liquordrainage handelt es sich um eine sehr individuelle Form der Ableitung. In einer Studie von West et al. (1987) zum ventrikulo-cholezystischen Shuntsystem wurden im Zeitraum von 1970 bis 1985 erstmals 25 Kinder im Alter von sechs Monaten bis 16 Jahre in einer Follow-up-Studie untersucht. Bei 80\% der Patienten war eine valide Auswertung möglich, bei der sich eine Komplikationsrate von 30\% zeigte. Operationsindikationen bei einem Shuntversagen waren zum Beispiel Infektion oder das Auftreten von Aszites. Ähnliche Ergebnisse zu dieser Thematik konnten Girotti et al. (2009) in einer kleinen Fallstudie mit fünf Patienten bestätigen und eine Erfolgsrate von 62,7\% für das Bestehen des ventrikulocholezystischen Shunts aufzeigen. Ebenso können Begleiterkrankungen, wie beispielsweise ein angeborener Plasminogenmangel in Kombination mit einem Hydrozephalus mit einem ventrikulo-cholezystischem Shunt versorgt werden. In denen vom Weinzierl et al. (2007) beschriebenen Fällen war dies als Möglichkeit nach zahlreichen erfolglosen Versuchen der VP und VA Ableitung als effiziente Ableitung möglich. Auch Demetriades et al. (2013) wählten bei zwei Patienten mit angeborenem Plasminogenmangel und multiplen Komplikationen bei vorherigen ventrikulo-peritonealen Ableitungen den Shunt in die Gallenblase als Drainagemöglichkeit. Alle Autoren waren sich einig, dass gerade nach einer Vielzahl stattgehabter erfolgloser Shuntversuche diese Behandlungsoption in Erwägung gezogen werden sollte (West et al. 1987; Weinzierl et al. 2007; Girotti et al. 2009).

In einem weiteren Fall war eine Patientin betroffen, welche als Frühgeborenes in der 36. SSW mit einer Meningomyelozele und assoziiertem HC versorgt wurde. Als Begleitkomplikation lag ein Faktor-V-Leiden vor, womit die Shuntmöglichkeit in das Gefäßsystem aufgrund der 
erhöhten Thrombosegefahr nicht gegeben war. Nach mehrmaligen abdominellen Liquorableitungsversuchen und dem Auftreten einer Liquorzyste erfolgte in Zusammenarbeit mit der Kinderchirurgie eine Umwandlung des Shuntsystems in eine ventrikulo-pleurale Ableitung. Dabei wird der Liquor in die Pleurahöhlen drainiert und dort resorbiert. Erstmals wurde diese Form der Ableitung 1954 von Joseph Ransohoff erfolgreich an einer sechsköpfigen Patientenreihe durchgeführt (Ransohoff 1954). In einer weiteren Follow-up-Studie von 1970 mit insgesamt 83 Kindern berichtete Ransohoff von einer Komplikationsrate in 35\% der Fälle. Das größte Risiko war dabei die Entstehung von Pleuraergüssen, da die Resorption des Liquors nicht gewährleistet werden konnte (Venes 1974). Hoffman et al. (1983) wiesen das Auftreten dieser Komplikation in $20 \%$ der von ihnen untersuchten Fälle nach (12 von 59 Patienten). Insbesondere waren von den betroffenen 12 Patienten neun erst fünf Jahre alt oder jünger. Auch Milhorat (1978) gibt an, dass von dieser Art der Shuntableitung bei Kindern unter drei Jahren und Patienten mit Lungenerkrankung Abstand genommen werden muss. Für den hier untersuchten Fall ist diese Schlussfolgerung jedoch zu vernachlässigen, da die Patientin zum Zeitpunkt des Eingriffs bereits 19 Jahre alt war. Daher entspricht die Entscheidung zur ventrikulo-pleuralen Ableitung aufgrund der mannigfaltigen Probleme der abdominellen Versuche und der fehlenden atrialen Ableitungsmöglichkeit den Erfahrungen in der Literatur. Bei postoperativen Pleuraergüssen hätte die Maßnahme noch weiter eskaliert werden können, indem das System auf eine bilaterale, pleurale Ableitung ausgeweitet wird (Ratliff et al. 2015).

Die beiden genannten Sonderfälle sind auch als Reserveoptionen bei multiplen Fehlversuchen der ventrikulo-peritonealen Ableitung in Betracht zu ziehen und insbesondere bei abdominellen Komplikationen nützlich. Bei Komorbidität der abdominellen Komplikationen ist der ventrikulo-pleurale Shunt dem ventrikulocholezystischem Shuntsystem vorzuziehen. Ferner sollen diese beiden doch sehr speziellen Fälle im Gesamtkollektiv noch einmal den Stellenwert der interdisziplinären Versorgung beim Krankheitsbild des kindlichen Hydrozephalus unterstreichen. Hinzukommend erfolgt über die beschriebene Zusammenarbeit bei der Problemstellung Shuntversorgung von Neurochirurgen und Kinderchirurgen der Universitätsmedizin Göttingen noch ein reger Austausch mit Kollegen aus anderen Abteilungen. Insbesondere die Kinderkardiologen sind in Bezug auf die Anlage von ventrikulo-atrialen Shuntsystemen involviert. In seltenen Fällen erfolgt auch eine Zusammenarbeit mit den Mitarbeitern aus dem Fachbereich der Urologie bei Shunts, welche in das ableitende Harnsystem Liquor drainieren. 


\subsubsection{Postoperativer Verlauf und Follow-up}

In Kapitel 3.4 wurde der Zeitraum nach der interdisziplinären Versorgung aufgearbeitet und dargestellt. Die Analyse der 61 Fälle ergab ein durchschnittliches interventionsfreies Intervall von 206 Tagen mit einer Spannweite von 1773 Tagen und einer Standardabweichung von 373 Tagen. Im Verlauf betrachtet waren bei $44 \%$ der Fälle eine erneute chirurgische Intervention innerhalb der ersten sechs Monate notwendig. Bei 21\% der Fälle war dies nach sechs Monaten der Fall. Die restlichen 34\% waren zum Zeitpunkt des Abschlusses der Datenerhebung interventionsfrei. Riva-Cambrin et al. (2015) beschreiben in ihrer Untersuchung ähnliche Zeitintervallen. Bei den auf Shuntversagen untersuchten 344 Fällen war das mittlere Shuntüberleben 344 Tage mit einer Spannweite von 1 - 932 Tagen (Bock et al. 2015). In einer anderen, breit angelegten Erhebung betrug das durchschnittliche Überleben 172 Tage (Shannon et al. 2013). Obwohl es eine Vielzahl von Studien zu der Thematik der Shuntüberlebensrate gibt, basieren nur wenige auf einem, wie dieser Dissertation zugrunde liegenden, selektierten Fallspektrum. In einer aktuellen Untersuchung befassen sich Burks et al. (2017) mit dem Outcome von Patienten nach abdominellen

Operationen und ventrikulo-peritonealer Shuntversorgung. Sie stellen die Hypothese auf, dass eine Shuntoperationen in einem Zeitfenster von 2 Wochen nach stattgehabter Bauchoperationen mit einem erhöhten Risiko für ein nachfolgendes Shuntversagen einhergeht. Gründe hierfür sind inflammatorische Reaktionen und das erhöhte Risiko für Adhäsionen.

Die Erfolgsraten zum Shuntüberleben konnten im Gegensatz zu den hier ausgewerteten Ergebnissen in anderen Studien als deutlich höher ausgewiesen werden. Die Hauptursache hierfür dürfte das sehr selektierte und in vielen Fällen sehr schwer kranke Kollektiv sein, welches eine interdisziplinäre Versorgung erst infolge des Krankheitsverlaufs notwendig machte. Insbesondere intraventrikuläre Hirnblutungen und Frühgeburtlichkeit habe wie bereits erörtert, einen negativen Einfluss auf die Erfolgsraten der Shuntbehandlungen (Shannon et al. 2012). 


\subsection{Vorteile und Nutzen einer interdisziplinären Versorgung}

Das Zustandekommen dieser Arbeit basiert vor allem auf der engen Zusammenarbeit von Kinderchirurgen und Neurochirurgen an der Universitätsmedizin Göttingen. Das Krankheitsbild des kindlichen Hydrozephalus ist nur ein Beispiel für eine Schnittstelle beider Fachdisziplinen. Das schon in Kapitel 2.1 beschriebene Operative Kinderzentrum (OPKIZ) bildet die Drehscheibe und Plattform für die Versorgung der Kinder.

Die Vorteile eine solchen Herangehensweise sind klar: Die moderne Medizin verlangt hohe Standards und die medizinischen Fälle werden immer komplexer. Durch die interdisziplinäre Versorgung werden Probleme aus unterschiedlichen Blickwinkeln beleuchtet. Dadurch werden auch schwierige Fälle greifbarer und die Fehlerquote sinkt (Ekenze et al. 2017). Dies ist auch bei vorliegendem Patientenkollektiv der Fall. Beispiele anderer Bereiche der Medizin, in denen ähnliche Arbeitsmodelle gelebt werden, sind Comprehensive Cancer Zentren oder Herzinsuffizienzzentren.

Im Zuge der Zusammenarbeit kann über einen ausreichend langen Zeitraum eine Prozessoptimierung erfolgen. Es können Standards und Leitlinien etabliert werden, die Transparenz und Effizienz schaffen, wie beispielsweise Winston und Dolan (2011) in ihrer Publikation über Shuntinfektionen herausarbeiten konnten. Das Göttinger Shuntregister ist ebenso ein Beispiel dafür, wie große Datenmengen greifbarer gemacht werden können (Bock et al. 2018b).

Auch der Faktor Mensch ist in dieser Gleichung eine sehr wichtige Variable. Die intraoperative Anwesenheit von Fachkollegen erleichtert die Entscheidungsfindung im Dialog und gibt gegenseitig Sicherheit. Gleichwohl ist der Lerneffekt gerade für junge Chirurgen sehr hoch, die von einem Austausch profitieren (Gillen et al. 2016).

Interdisziplinäre Zusammenarbeit ist die Voraussetzung für die Einheit von Krankenversorgung, Lehre und Forschung. Das Krankheitsbild des kindlichen Hydrozephalus ist zu komplex, als dass es nur noch von einer Fachdisziplin versorgt werden könnte. Im selben Atemzug gilt es auch die interprofessionelle Arbeit hervorzuheben, denn ohne das Mitwirken von Pflegepersonal, Sozialpädiatrischem Dienst und vielen weiteren Beteiligten wäre eine angemessene Behandlung der Kinder nicht denkbar. 


\section{$5 \quad$ Fazit}

In dieser Arbeit wurde anhand einer repräsentativen Stichprobe ein Überblick über die interdisziplinäre Behandlung von abdominellen Komplikationen bei Kindern mit ventrikuloperitonealem Shuntsystem versorgten Hydrozephalus gegeben. Dabei wurde das vorliegende Fallkollektiv auf Risikofaktoren untersucht und die genauen Schnittpunkte der Zusammenarbeit von Kinderchirurgie und Neurochirurgie erarbeitet. Trotz des repräsentativen Patientenquerschnitts muss angemerkt werden, dass die Stichprobengröße mit 61 Fällen klein und die wissenschaftlichen Aussagemöglichkeiten daher begrenzt sind.

Nach der Risikofaktorenanalyse ließ sich schlussfolgern, dass ein hoher Anteil der Fälle einen posthämorrhagischen Hydrozephalus als Ursache für eine notwendige VPS-Versorgung hatte. Dies stellt somit einen möglichen Faktor für die Entwicklung von abdominellen Komplikationen bei stattgehabter Anlage eines ventrikulo-peritonealen Shuntsystems dar. Darüber hinaus sollte eine einmal begonnene interdisziplinäre Zusammenarbeit auch nach Versorgung des Kindes fortgeführt werden.

Das Vorliegen einer nekrotisierenden Enterokolitis in der Anamnese ist ebenso als Ursache für abdominelle Shuntkomplikationen in Betracht zu ziehen. Gleiches gilt für frühgeborene Kinder, welche einen Großteil des Fallkollektivs ausmachen und nicht nur insgesamt anfälliger für postpartale Komplikationen sind, sondern auch abdominelle Probleme nach ventrikulo-peritonealer Shuntanlage begünstigen.

Das Geburtsgewicht der Kinder ist weit gestreut und im Mittel deutlich unter den in Göttingen zur Shuntanlage notwendigen 2500 g. Eine genaue Aussage über den Einfluss des Gewichts auf das Auftreten von abdominellen Komplikationen kann auf Grundlage des hier untersuchten Kollektivs nicht getroffen werden, dieser ist aber in Zusammenschau mit der aktuellen Literatur sehr wahrscheinlich.

Im Gegensatz dazu lassen sich keine Schlussfolgerungen über eine Relevanz von Infektionen als Risikofaktor für die Entwicklung abdomineller Komplikationen bei vorhandenem VPS ziehen. Dennoch sei an dieser Stelle noch einmal betont, dass Infektionen in Bezug auf allgemeine Shuntkomplikationen enorme Bedeutung haben.

In dem postoperativen Follow-up ist eine hohe Anfälligkeit für Folgekomplikationen aufgetreten. Insgesamt wurden $44 \%$ der Fälle innerhalb von 6 Monaten nach interdisziplinärer Versorgung erneut revidiert, allerdings auch außerhalb der interdisziplinären Indikationen, da wachstumsbedingte Komplikationen, der höhere 
hydrostatische Effekt mit zunehmender Körpergröße und migratorische Komponenten der Shuntanteile eine fortwährende Rolle im Kindes- und Jugendalter spielen. Damit ist die Shuntüberlebensrate geringer als im Gesamtkollektiv des Göttinger Shuntregisters, welches sich am ehesten mit der Multimorbidität des vorliegenden Fallkollektivs erklären lässt.

Betrachtet man die einzelnen Interventionsnotwendigkeiten der Fachdisziplinen separat, dann fällt auf, dass die Kinderchirurgie hauptsächlich bei voroperierten Bauchhöhlen tätig werden musste. Dabei wurden vor allem Adhäsiolysen von intraperitonealen Verwachsungen durchgeführt. Die Hauptoperationsindikationen der Neurochirurgen waren Shuntrevisionen und Shuntneuanlagen.

Die dargestellten Zusammenhänge und gerade das Vorhandensein einer oder mehrerer der oben genannten Risikofaktoren rechtfertigen die Aussage, dass eine fachübergreifende Zusammenarbeit nicht nur sinnvoll ist und dem Wohl des Patienten dient, sondern in dem beschriebenen Patientenkollektiv mandatorisch ist. Präoperativ sollte eine Analyse der in dieser Dissertation erhobenen Risikofaktoren durchgeführt werden. Insbesondere abdominelle Komplikationen nach ventrikulo-peritonealer Shuntanlage bedürfen der Aufmerksamkeit von Neurochirurgen und Kinderchirurgen. 


\section{Ausblick}

Die Tatsache, dass eine kurative Versorgung des Krankheitsbildes des kindlichen Hydrozephalus nur bedingt bis gar nicht möglich ist, führt zu der Schlussfolgerung, dass in erster Linie alles versucht werden sollte, eine Shuntanlage zu verhindern. Dies beinhaltet insbesondere Weiterentwicklungen in der Neonatologie und Pädiatrie und im Detail die Vermeidung von intraventrikulären Hirnblutungen. Darüber hinaus ist es insbesondere für die in dieser Arbeit speziell behandelte Fallklientel relevant, die Prävention für das Auftreten der nekrotisierenden Enterokolitis bei Frühgeborenen weiter zu verbessern (Lawn et al. 2014).

Sind irreparable Schäden durch einen bestehenden Hydrozephalus bei nicht sistierenden Hirndruckerhöhungen unvermeidbar, dann ist die Therapie und der Zeitpunkt der Versorgung sehr sorgfältig zu planen. Nach Tuli et al. (2000) ist eine möglichst späte Shuntanlage für ein positives Outcome der Kinder günstig. Als weitere effektive und kurative Behandlungsoption neben der klassischen Shuntanlage hat in den letzten Jahren die ETV (endoscopic third ventriculotomy) immer mehr an Bedeutung gewonnen. Die eigentliche Behandlungsindikation bei Nicht-kommunizierendem Hydrozephalus wurde auf den kommunizierenden Hydrozephalus erweitert (Hailong et al. 2008; Singh et al. 2008; RangelCastilla et al. 2012). Zusätzlich führte die Kombination der ETV mit der Plexus Kauterisation zu einer Verbesserung des Patienten-Outcomes (Warf 2013; Chamiraju et al. 2014; Shitsama et al. 2014). In Bezug auf die Erfolgsrate unterschiedlicher Behandlungsoptionen ist festzuhalten, dass Shunt- oder endoskopische Behandlungen nach wie vor Gegenstand wissenschaftlicher Auseinandersetzungen sind und es in Studien zu unterschiedlichen Ergebnissen und Aussagen kommt. Erste Daten erlangten Kulkarni et al. (2016) in ihrer prospektiven Studie, welche 2004 begonnen hat. Es wurden insgesamt 182 Kindern unter 2 Jahren mit Hydrozephalus aufgrund einer Aquäduktstenose untersucht, die entweder mit Shunt oder ETV versorgt wurden. In den noch nicht endgültigen abgeschlossenen Auswertungen zeigen sich bereits hohe Erfolgsraten bei beiden Methoden mit einem Vorteil zugunsten der Shunttherapie (Nach 36 Monate Erfolgsrate ETV $=64,1 \%$ Shunt $=79 ., 1 \%$ ). Die ETV ist insbesondere bei Kindern unter 6 Monaten mit einem erhöhten Misserfolgsrisiko assoziiert (Kulkarni et al. 2016).

Die Behandlungsoptionen bei abdominellen Symptomen nach VPS Versorgung sind komplex und es existieren momentan keine Leitlinien zu einheitlichen Behandlung. Diese fehlende Stütze sollte in naher Zukunft erarbeitet werden. 
Neben therapeutischen und Leitlinien-orientierten Verbesserungen gibt es auch Verbesserungspotential bei technischen Komponenten, welches durch die Einführung von Antibiotika-beschichteten Shunts, programmierbaren Ventilen und Antisiphongeräten bereits genutzt wird. In Zukunft könnten Smart-Devices Übersicht über Liquorabflussraten und Echtzeithirndrücke geben, eine Entwicklung, die völlig neue Therapieoptionen eröffnet (Lutz et al. 2013). Weiterhin sind ähnlich wie in der Kardiologie genutzte, medikamentenbeschichtete, distale Shuntsysteme in der Entwicklung und sollen einem Einwachsen des peritonealen Schenkels vorbeugen. Dies könnte insbesondere die Rate der distalen Shuntobstruktionen minimieren (Harris und McAllister 2012).

Obwohl die in dieser Dissertation im Fokus stehende Thematik bereits Gegenstand der Literatur ist, existieren bisher wenig weiterführende Untersuchungen oder umfassende wissenschaftliche Studien zur Thematik der abdominellen Komplikationen nach ventrikuloperitonealer Shuntanlage bei Kindern. In den vergangenen 10-15 Jahren gab es im Forschungsfeld um den kindlichen Hydrozephalus einen Übergang von kleinen Fallstudien hin zu größer angelegten prospektiven Studien. Die Einführung von Shuntregistern, wie dem UK Shunt Registry, dem Hydrocephalus Clinical Research Network (HCRN) und Datenbanken wie beispielsweise dem hier genutzten Göttinger Shuntregister (Bock et al. 2018b), hat die wissenschaftliche Grundlage für die weitere, vertiefende und standardisierte Forschung geschaffen. Diese Erfolge lassen sich vor allem anhand der in der Vergangenheit gesunkenen Infektionsraten und verbesserten Shuntüberlebensraten messen. Die weitere Verbreitung dieser Datenerfassungssysteme könnte die Vergleichbarkeit zwischen verschiedenen Krankenhäusern erleichtern und die Transparenz erhöhen. Neben den statistischen Daten sollte auch am grundlegenden pathophysiologischen Modell des Hydrozephalus weiter geforscht werden, um darauf aufbauend neue therapeutische Strategien entwickeln zu können (Kahle et al. 2016). 


\section{$7 \quad$ Literaturverzeichnis}

de Aquino HB, Carelli EF, Neto AGB, Pereira CU (2006): Nonfunctional abdominal complications of the distal catheter on the treatment of hydrocephalus: an inflammatory hypothesis? Childs Nerv Syst 22, 1225-1230

Aryan HE, Meltzer HS, Park MS, Bennett RL, Jandial R, Levy ML (2005): Initial experience with antibiotic-impregnated silicone catheters for shunting of cerebrospinal fluid in children. Childs Nerv Syst $\underline{21}, 56-61$

Aschoff A, Kremer P, Hashemi B, Kunze S (1999): The scientific history of hydrocephalus and its treatment. Neurosurg Rev 22, 67-93

Asmerom M, Crowe L, Marin T (2015): Understanding the Biologic Therapies of Probiotics, Prebiotics, and Synbiotics: Exploring Current Evidence for Use in Premature Infants for the Prevention of Necrotizing Enterocolitis. J Perinat Neonatal Nurs 29, 240247

Baird C, O’Connor D, Pittman T (1999): Late shunt infections. Pediatr Neurosurg $\underline{31}$, 269-273

Baird LC, Mazzola CA, Auguste KI, Klimo P, Flannery AM (2014): Pediatric hydrocephalus: systematic literature review and evidence-based guidelines. Part 5: Effect of valve type on cerebrospinal fluid shunt efficacy. J Neurosurg Pediatr $\underline{14}$, 35-43

Ballabh P (2010): Intraventricular Hemorrhage in Premature Infants: Mechanism of Disease. Pediatr Res $\underline{67}, 1-8$

Barina AR, Virgo KS, Mushi E, Bahadursingh AM, Johnson FE (2007): Appendectomy for appendicitis in patients with a prior ventriculoperitoneal shunt. J Surg Res $\underline{141}, 40$ 44

Beez T, Sarikaya-Seiwert S, Bellstädt L, Mühmer M, Steiger H-J (2014): Role of ventriculoperitoneal shunt valve design in the treatment of pediatric hydrocephalus - a single center study of valve performance in the clinical setting. Childs Nerv Syst $\underline{30}, 293-$ 297

Bernardo WM, Aires FT, Carneiro RM, de Sá FP, Rullo VEV, Burns DA (2013): Effectiveness of probiotics in the prophylaxis of necrotizing enterocolitis in preterm neonates: a systematic review and meta-analysis. J Pediatr (Rio J) 89, 18-24

Del Bigio MR, Di Curzio DL (2016): Nonsurgical therapy for hydrocephalus: a comprehensive and critical review. Fluids Barriers CNS 13, 3

Bilginer B, Oguz KK, Akalan N (2009): Endoscopic third ventriculostomy for malfunction in previously shunted infants. Childs Nerv Syst 25, 683-688

Bir SC, Konar S, Maiti TK, Kalakoti P, Bollam P, Nanda A (2016): Outcome of ventriculoperitoneal shunt and predictors of shunt revision in infants with 
posthemorrhagic hydrocephalus. Childs Nerv Syst $\underline{32}$, 1405-1414

Blackburn BL, Fineman RM (1994): Epidemiology of congenital hydrocephalus in Utah, 1940-1979: report of an iatrogenically related „epidemic“. Am J Med Genet 52, 123-129

Blakely ML, Lally KP, McDonald S, Brown RL, Barnhart DC, Ricketts RR, Thompson WR, Scherer LR, Klein MD, Letton RW, et al. (2005): Postoperative Outcomes of Extremely Low Birth-Weight Infants With Necrotizing Enterocolitis or Isolated Intestinal Perforation. Ann Surg 241, 984-994

Blencowe H, Cousens S, Chou D, Oestergaard M, Say L, Moller A-B, Kinney M, Lawn J (2013): Born Too Soon: The global epidemiology of 15 million preterm births. Reprod Health $\underline{10}$, S2

Bock HC, Kanzler M, Ludwig HC: Implementation of a CSF-shunt-registry for monitoring clinical follow-up and to evaluate long-term outcome in paediatric hydrocephalus patients.; 66th Annual Meeting of the German Society of Neurosurgery (DGNC), Karlsruhe 2015, Meeting Abstract

Bock HC, Feldmann J, Ludwig HC (2018a): Early surgical management and long-term surgical outcome for intraventricular hemorrhage-related posthemorrhagic hydrocephalus in shunt-treated premature infants. J Neurosurg Pediatr $\underline{22}, 61-67$

Bock HC, Kanzler M, Thomale U-W, Ludwig HC (2018b): Implementing a digital realtime Hydrocephalus and Shunt Registry to evaluate contemporary pattern of care and surgical outcome in pediatric hydrocephalus. Childs Nerv Syst 34, 457-464

Bouras T, Sgouros S (2011): Complications of endoscopic third ventriculostomy. J Neurosurg Pediatr $\underline{7}, 643-649$

Browd SR, Ragel BT, Gottfried ON, Kestle JRW (2006a): Failure of Cerebrospinal Fluid Shunts: Part I: Obstruction and Mechanical Failure. Pediatr Neurol 34, 83-92

Browd SR, Gottfried ON, Ragel BT, Kestle JRW (2006b): Failure of Cerebrospinal Fluid Shunts: Part II: Overdrainage, Loculation, and Abdominal Complications. Pediatr Neurol 34, 171-176

Brunelle F: Modern Imaging of Pediatric Hydrocephalus.; in: Pediatric Hydrocephalus; hrsg. v. Cinalli G, Sainte-Rose C, Maixner WJ; Springer, Mailand 2005, 79-93

Burgmeier C, Schier F (2016): The Role of Laparoscopy in the Acute Neonatal Abdomen. Surg Innov 23, 635-639

Burhan B, Serdar KB, Abdurrahman A, Edip AM, Ebuzer D (2018): Abdominal Complications of Ventriculoperitoneal Shunt in Pediatric Patients: Experiences of a Pediatric Surgery Clinic. World Neurosurg 118, e129-e136

Burks JD, Conner AK, Briggs RG, Glenn CA, Bonney PA, Cheema AA, Chen S, Gross NL, Mapstone TB (2017): Risk of failure in pediatric ventriculoperitoneal shunts placed after abdominal surgery. J Neurosurg Pediatr $\underline{19}, 571-577$ 
Callaghan WM, Macdorman MF, Shapiro-Mendoza CK, Barfield WD (2016): Explaining the recent decrease in US infant mortality rate, 2007-2013. Am J Obstet Gynecol 216, 73.e1-73.e8

Carvalho FO de, Bellas AR, Guimarães L, Salomão JF, Carvalho FO de, Bellas AR, Guimarães L, Salomão JF (2014): Laparoscopic assisted ventriculoperitoneal shunt revisions as an option for pediatric patients with previous intraabdominal complications. Arq Neuropsiquiatr $\underline{72}, 307-311$

Çelik A, Ergün O, Arda MS, Yurtseven T, Erşahin Y, Balik E (2005): The incidence of inguinal complications after ventriculoperitoneal shunt for hydrocephalus. Childs Nerv Syst $\underline{21}, 44-47$

Chamiraju P, Bhatia S, Sandberg DI, Ragheb J (2014): Endoscopic third ventriculostomy and choroid plexus cauterization in posthemorrhagic hydrocephalus of prematurity. $\mathrm{J}$ Neurosurg Pediatr $\underline{13}$, 433-439

Chen Y-C, Wu J-C, Liu L, Chen T-J, Huang W-C, Cheng H (2011): Correlation between ventriculoperitoneal shunts and inguinal hernias in children: an 8-year follow-up. Pediatrics $\underline{128}$, e121-e126

Cherian S, Whitelaw A, Thoresen M, Love S (2004): The Pathogenesis of Neonatal Posthemorrhagic Hydrocephalus. Brain Pathol 14, 305-311

Chittiboina P, Pasieka H, Sonig A, Bollam P, Notarianni C, Willis BK, Nanda A (2013): Posthemorrhagic hydrocephalus and shunts: what are the predictors of multiple revision surgeries? J Neurosurg Pediatr 11, 37-42

Chung J-J, Yu J-S, Kim JH, Nam SJ, Kim M-J (2009): Intraabdominal Complications Secondary to Ventriculoperitoneal Shunts: CT Findings and Review of the Literature. Am J Roentgenol 193, 1311-1317

Cinalli G, Cappabianca P, de Falco R, Spennato P, Cianciulli E, Cavallo LM, Esposito F, Ruggiero C, Maggi G, de Divitiis E (2005): Current state and future development of intracranial neuroendoscopic surgery. Expert Rev Med Devices 2, 351-373

Clarnette TD, Lam SK, Hutson JM (1998): Ventriculo-peritoneal shunts in children reveal the natural history of closure of the processus vaginalis. J Pediatr Surg $\underline{33}, 413-$ 416

Collins P, Hockley AD, Woollam DH (1978): Surface ultrastructure of tissues occluding ventricular catheters. J Neurosurg $\underline{48}, 609-613$

Cutts S, Talboys R, Paspula C, Ail D, Premphe EM, Fanous R (2016): History of acute respiratory distress syndrome. Lancet Respir Med 4, 547-548

Dabdoub CB, Dabdoub CF, Chavez M, Villarroel J, Ferrufino JL, Coimbra A, Orlandi BM (2014): Abdominal cerebrospinal fluid pseudocyst: a comparative analysis between children and adults. Childs Nerv Syst $\underline{30}, 579-589$ 
Dandy W, Blackfan K (1914): An experimental, clinical and pathological study: Part 1.experimental studies. Am J Dis Child VIII, 406-482

Davidson AJ, Disma N, de Graaff JC, Withington DE, Dorris L, Bell G, Stargatt R, Bellinger DC, Schuster T, Arnup SJ, et al. (2016): Neurodevelopmental outcome at 2 years of age after general anaesthesia and awake-regional anaesthesia in infancy (GAS): an international multicentre, randomised controlled trial. Lancet $\underline{387}, 239-250$

Decq P, Schroeder HWS, Fritsch M, Cappabianca P (2013): A History of Ventricular Neuroendoscopy. World Neurosurg $\underline{79}$, S14.e1-S14.e6

Deeg KH, Staudt F, von Rohden L (1999): Klassifikation der intrakraniellen Blutungen des Frühgeborenen. Ultraschall Med 20, 165-170

Del Bigio MR (1998): Biological reactions to cerebrospinal fluid shunt devices: a review of the cellular pathology. Neurosurgery $\underline{42}, 319-326$

Dewan MC, Rattani A, Mekary R, Glancz LJ, Yunusa I, Baticulon RE, Fieggen G, Wellons JC, Park KB, Warf BC (2018): Global hydrocephalus epidemiology and incidence: systematic review and meta-analysis. J Neurosurg 130, 1065-1079

Drake JM, Kestle JR, Milner R, Cinalli G, Boop F, Piatt J, Haines S, Schiff SJ, Cochrane DD, Steinbok P, MacNeil N (1998): Randomized trial of cerebrospinal fluid shunt valve design in pediatric hydrocephalus. Neurosurgery $\underline{43}, 294-305$

Drake JM, Kestle JRW, Tuli S (2000): CSF shunts 50 years on - past, present and future. Childs Nerv Syst $\underline{16}$, 800-804

Eckardstein KL von, Kallenberg K, Psychogios M-N, Schatlo B, Rohde V, Ludwig HC, Bock HC (2017): Contrast-enhanced shunt series ("shuntography") compare favorably to other shunt imaging modalities in detecting shunt occlusion. Acta Neurochir (Wien) 159 , $63-70$

Eichenwald EC, Stark AR (2008): Management and Outcomes of Very Low Birth Weight. N Engl J Med 358, 1700-1711

Ekenze SO, Modekwe VO, Ajuzieogu OV, Asinobi IO, Sanusi J (2017): Neonatal surgery in a developing country: Outcome of co-ordinated interdisciplinary collaboration. $\mathrm{J}$ Paediatr Child Health 53, 976-980

Elvidge AR, Branch CL, Thompson GB (1957): Observations in a Case of Hydrocephalus Treated with Diamox. J Neurosurg 14, 628-639

Fenton TR, Kim JH (2013): A systematic review and meta-analysis to revise the Fenton growth chart for preterm infants. BMC Pediatr 13, 59

Fitzgibbons SC, Ching Y, Yu D, Carpenter J, Kenny M, Weldon C, Lillehei C, Valim C, Horbar JD, Jaksic T (2009): Mortality of necrotizing enterocolitis expressed by birth weight categories. J Pediatr Surg $\underline{44}, 1072-1076$ 
Flannery AM, Mitchell L (2014): Pediatric hydrocephalus: systematic literature review and evidence-based guidelines. Part 1: Introduction and methodology. J Neurosurg Pediatr 14 Suppl 1, 3-7

Ford H, Watkins S, Reblock K, Rowe M (1997): The role of inflammatory cytokines and nitric oxide in the pathogenesis of necrotizing enterocolitis. J Pediatr Surg $\underline{32}$, 275-282

Fritsch MJ, Schroeder HWS (2013): Endoscopic Aqueductoplasty and Stenting. World Neurosurg 79, S20.e15-S20.e18

Furlanetti LL, Santos MV, de Oliveira RS (2012): The success of endoscopic third ventriculostomy in children: analysis of prognostic factors. Pediatr Neurosurg $\underline{48}, 352-$ 359

Garbi-Goutel A, Brévaut-Malaty V, Panuel M, Michel F, Merrot T, Gire C (2014): Prognostic value of abdominal sonography in necrotizing enterocolitis of premature infants born before 33 weeks gestational age. J Pediatr Surg 49, 508-513

Garton HJ, Kestle JR, Drake JM (2001): Predicting shunt failure on the basis of clinical symptoms and signs in children. J Neurosurg 94, 202-210

Gaskill SJ, Marlin AE (1989): Pseudocysts of the abdomen associated with ventriculoperitoneal shunts: a report of twelve cases and a review of the literature. Pediatr Neurosci 15, 23-26

Geng J, Wu D, Chen X, Zhang M, Xu B, Yu X (2015): Aqueduct Stent Placement: Indications, Technique, and Clinical Experience. World Neurosurg $\underline{84}, 1347-1353$

Gillen JR, Ramirez AG, Farineau DW, Hoke TR, Schirmer BD, Williams MD, Lau CL (2016): Using Interdisciplinary Workgroups to Educate Surgery Residents in SystemsBased Practice. J Surg Educ 73, 1052-1059

Girotti ME, Singh RR, Rodgers BM (2009): The Ventriculo-Gallbladder Shunt in the Treatment of Refractory Hydrocephalus: A Review of the Current Literature. Am Surg $\underline{75}, 734-737$

Grant HW, Parker MC, Wilson MS, Menzies D, Sunderland G, Thompson JN, Clark DN, Knight AD, Crowe AM, Ellis H (2008): Adhesions after abdominal surgery in children. J Pediatr Surg $\underline{43}, 152-157$

Gratzl O, Merlo A: Hydrozephalus.; in: Chirurgie., 8. Auflage; hrsg. v. Siewert JR; Springer, Berlin 2006, 229-231

Greitz D (2007): Paradigm shift in hydrocephalus research in legacy of Dandy's pioneering work: rationale for third ventriculostomy in communicating hydrocephalus. Childs Nerv Syst $\underline{23}, 487$

Greitz D, Greitz T, Hindmarsh T (1997): A new view on the CSF-circulation with the potential for pharmacological treatment of childhood hydrocephalus. Acta Pædiatrica $\underline{86}$, $125-132$ 
International PHVD Drug Trial Group (1998): International randomised controlled trial of acetazolamide and furosemide in posthaemorrhagic ventricular dilatation in infancy. Lancet $352,433-440$

Gurtner P, Bass T, Gudeman SK, Penix JO, Philput CB, Schinco FP (1992): Surgical management of posthemorrhagic hydrocephalus in 22 low-birth-weight infants. Childs Nerv Syst $\underline{8}, 198-202$

Hadani M, Findler G, Muggia-Sullam M, Sahar A (1982): Acute appendicitis in children with a ventriculoperitoneal shunt. Surg Neurol 18, 69-71

Hailong F, Guangfu H, Haibin T, Hong P, Yong C, Weidong L, Dongdong Z (2008): Endoscopic third ventriculostomy in the management of communicating hydrocephalus: a preliminary study. J Neurosurg $\underline{109}, 923-930$

Harris CA, McAllister JP (2012): What We Should Know About the Cellular and Tissue Response Causing Catheter Obstruction in the Treatment of Hydrocephalus. Neurosurgery $\underline{70}, 1589-1602$

Harsh GR (1954): Peritoneal shunt for hydrocephalus, utilizing the fimbria of the fallopian tube for entrance to the peritoneal cavity. J Neurosurg 11, 284-294

Hashimoto M, Yokota A, Urasaki E, Tsujigami S, Shimono M (2004): A case of abdominal CSF pseudocyst associated with silicone allergy. Childs Nerv Syst 20, 761764

He M, Ouyang L, Wang S, Zheng M, Liu A (2016): Laparoscopy versus mini-laparotomy peritoneal catheter insertion of ventriculoperitoneal shunts: a systematic review and metaanalysis. Neurosurg Focus $\underline{41}$, E7

Hoffman HJ, Hendrick B, Humphreys RP (1983): Experience with Ventriculo-pleural Shunts. Pediatr Neurosurg $\underline{10}, 404-413$

Hollingsworth CL, Rice HE (2010): The Duke Abdominal Assessment Scale: initial experience. Expert Rev Gastroenterol Hepatol 4, 569-574

Horwitz JR, Lally KP, Cheu HW, Vazquez WD, Grosfeld JL, Ziegler MM (1995): Complications after surgical intervention for necrotizing enterocolitis: a multicenter review. J Pediatr Surg 30, 994-999

Horwitz NH (1997): Walter Edward Dandy (1886-1946). Neurosurgery 40, 211-215

Jaiswal AK, Jaiswal S, Sahu RN, Das KB, Jain VK, Behari S (2009): Choroid plexus papilloma in children: Diagnostic and surgical considerations. J Pediatr Neurosci $\underline{4}, 10$ 16

Jansen O, Ebinger F, Stephani U, Deuschl G, Diener H-C, Hopf HC, Reichmann H (Hrsg.): Fehlbildungen und frühkindliche Schädigungen des ZNS. (RRN - ReferenzReihe Neurologie), 1. Aufl.; Georg Thieme Verlag, Stuttgart 2007 
Johnson BW, Pimpalwar A (2009): Laparoscopic-assisted placement of ventriculoperitoneal shunt tips in children with multiple previous open abdominal ventriculoperitoneal shunt surgeries. Eur J Pediatr Surg 19, 79-82

Jones RF, Stening WA, Brydon M (1990): Endoscopic third ventriculostomy. Neurosurgery $\underline{26}, 86-91$

Kahle KT, Kulkarni AV, Limbrick DD, Warf BC (2016): Hydrocephalus in children. Lancet $\underline{387}, 788-799$

Kargl S, Wagner O, Pumberger W (2017): Ileostomy Complications in Infants less than 1500 grams - Frequent but Manageable. J Neonatal Surg $\underline{6}, 4$

Kellie G (1824): An account of the appearances observed in the dissection of two of three individuals presumed to have perished in the storm of the $3 \mathrm{rd}$, and whose bodies were discovered in the vicinity of Leith on the morning of the 4th, November 1821; with some reflections on the pathology of the brain. Trans Med Chir Soc 1, , 84-122

Kestle J, Drake J, Milner R, Sainte-Rose C, Cinalli G, Boop F, Piatt J, Haines S, Schiff S, Cochrane D, et al. (2000): Long-term follow-up data from the Shunt Design Trial. Pediatr Neurosurg 33, 230-236

Key CB, Rothrock SG, Falk JL (1995): Cerebrospinal fluid shunt complications: an emergency medicine perspective. Pediatr Emerg Care 11, 265-273

Khan MB, Riaz M, Enam SA (2016): Endoscopic third ventriculostomy for obstructive hydrocephalus: Outcome analysis of 120 consecutively treated patients from a developing country. Int J Surg 26, $69-72$

Kirsch J, May CA, Lorke D, Winkelmann A, Schwab W, Herrmann G, Funk R (Hrsg.): Taschenlehrbuch Anatomie. Thieme, Stuttgart 2011

Klimo P, Thompson CJ, Baird LC, Flannery AM, Pediatric Hydrocephalus Systematic Review and Evidence-Based Guidelines Task Force (2014): Pediatric hydrocephalus: systematic literature review and evidence-based guidelines. Part 7: Antibioticimpregnated shunt systems versus conventional shunts in children: a systematic review and meta-analysis. J Neurosurg Pediatr 14 Suppl 1, 53-59

Köksal V, Öktem S (2010): Ventriculosubgaleal shunt procedure and its long-term outcomes in premature infants with post-hemorrhagic hydrocephalus. Childs Nerv Syst $\underline{26}, 1505-1515$

Kulkarni AV, Sgouros S, Constantini S, Investigators I (2016): International Infant Hydrocephalus Study: initial results of a prospective, multicenter comparison of endoscopic third ventriculostomy (ETV) and shunt for infant hydrocephalus. Childs Nerv Syst $\underline{32}, 1039-1048$

Lawn JE, Blencowe H, Oza S, You D, Lee AC, Waiswa P, Lalli M, Bhutta Z, Barros AJ, Christian P, et al. (2014): Every Newborn: progress, priorities, and potential beyond 
survival. Lancet 384, 189-205

Leibrock L, Baker R, Uematsu S (1976): Simulated acute appendicitis secondary to ventriculoperitoneal shunt. Surg Neurol $\underline{5}, 105-107$

Lifshutz JI, Johnson WD (2001): History of hydrocephalus and its treatments. Neurosurg Focus $\underline{11}, 1-5$

Limbrick DD, Baird LC, Klimo P, Riva-Cambrin J, Flannery AM, Pediatric Hydrocephalus Systematic Review and Evidence-Based Guidelines Task Force (2014): Pediatric hydrocephalus: systematic literature review and evidence-based guidelines. Part 4: Cerebrospinal fluid shunt or endoscopic third ventriculostomy for the treatment of hydrocephalus in children. J Neurosurg Pediatr 14 Suppl 1, 30-34

Lippert H: Lehrbuch Anatomie. 7., erw. Aufl; Elsevier, Urban und Fischer, München 2006

Ludwig HC, Kruschat T, Knobloch T, Teichmann H-O, Rostasy K, Rohde V (2007): First experiences with a 2.0- $\mu \mathrm{m}$ near infrared laser system for neuroendoscopy. Neurosurg Rev 30, 195-201

Lumenta CB, Rocco CD, Haase J: Neurosurgery. Springer, Berlin 2009

Lutz BR, Venkataraman P, Browd SR (2013): New and improved ways to treat hydrocephalus: Pursuit of a smart shunt. Surg Neurol Int $\underline{4}$, S38-S50

Major O, Fedorcsák I, Sipos L, Hantos P, Kónya E, Dobronyi I, Paraicz E (1994): Slitventricle syndrome in shunt operated children. Acta Neurochir (Wien) 127, 69-72

Kofoed Månsson P, Johansson S, Ziebell M, Juhler M (2017): Forty years of shunt surgery at Rigshospitalet, Denmark: a retrospective study comparing past and present rates and causes of revision and infection. BMJ Open $\underline{7}$, e013389

Marton E, Feletti A, Basaldella L, Longatti P (2010): Endoscopic third ventriculostomy in previously shunted children: a retrospective study. Childs Nerv Syst $\underline{26}, 937-943$

Mazzola CA, Choudhri AF, Auguste KI, Limbrick DD, Rogido M, Mitchell L, Flannery AM, Pediatric Hydrocephalus Systematic Review and Evidence-Based Guidelines Task Force (2014): Pediatric hydrocephalus: systematic literature review and evidence-based guidelines. Part 2: Management of posthemorrhagic hydrocephalus in premature infants. J Neurosurg Pediatr 14 Suppl 1, 8-23

McCullough DC (1986): A History of the Treatment of Hydrocephalus. Fetal Diagn Ther $1,38-45$

Milhorat TH: Pediatric neurosurgery. F. A. Davis, Philadelphia 1978

Mobley LW, Doran SE, Hellbusch LC (2005): Abdominal pseudocyst: predisposing factors and treatment algorithm. Pediatr Neurosurg $\underline{41}, 77-83$

Monro A: Observations on the structure and functions of the nervous system: illustrated 
with tables. Creech, Edinburgh 1783

Morello G, Migliavacca F (1959): Third ventriculostomy (Stookey and Scarff operation) in the treatment of benign aqueductal stenosis. Acta Neurochir (Wien) $\underline{7}, 417-424$

Morishita A, Nagashima T, Kurata H, Eguchi T, Tamaki N (2002): Clinical analysis of pediatric shunt catheter fracture. No Shinkei Geka $\underline{30}$, 839-845

Morosanu CO, Filip GA, Nicolae L, Florian IS (2018): From the heart to the bladderparticularities of ventricular shunt topography and the current status of cerebrospinal fluid diversion sites. Neurosurg Rev 10.1007/s10143-018-1033-2

Murshid WR, Jarallah JS, Dad MI (2000): Epidemiology of infantile hydrocephalus in Saudi Arabia: birth prevalence and associated factors. Pediatr Neurosurg 32, 119-123

Muthukumar N, Venkatesh G, Thiruppathy S (2005): Arrested hydrocephalus and the presyrinx state. J Neurosurg Pediatr 103, 466-470

Narendra A, White M, Rolton H, Alloub Z, Wilkinson G, McColl J, Beattie J (2001): Nephrocalcinosis in preterm babies. Arch Dis Child Fetal Neonatal Ed 85, F207-F213

Neiter E, Guarneri C, Pretat P-H, Joud A, Marchal J-C, Klein O (2016): Semiology of ventriculoperitoneal shunting dysfunction in children - a review. Neurochirurgie $\underline{62}, 53-$ 59

Neu J (2014): Necrotizing Enterocolitis: The Mystery Goes On. Neonatology 106, 289_ 295

Neu J, Walker WA (2011): Necrotizing Enterocolitis. N Engl J Med 364, 255-264

Nigim F, Thomas AJ, Papavassiliou E, Schneider BE, Critchlow JF, Chen CC, Siracuse JJ, Zinn PO, Kasper EM (2014): Ventriculoperitoneal shunting: Laparoscopically assisted versus conventional open surgical approaches. Asian J Neurosurg $\underline{9}, 72$

Nulsen FE, Spitz EB (1951): Treatment of hydrocephalus by direct shunt from ventricle to jugular vain. Surg Forum 399-403

Ohmann C, Franke C, Kraemer M, Yang Q (2002): Neues zur Epidemiologie der akuten Appendizitis. Chirurg 73, 769-776

Oi S (2003): Diagnosis, outcome, and management of fetal abnormalities: fetal hydrocephalus. Childs Nerv Syst $\underline{19}, 508-516$

Oi S (2011): Classification of hydrocephalus: critical analysis of classification categories and advantages of „Multi-categorical Hydrocephalus Classification“ (Mc HC). Childs Nerv Syst 27, 1523-1533

Orešković D, Klarica M (2010): The formation of cerebrospinal fluid: Nearly a hundred years of interpretations and misinterpretations. Brain Res Rev 64, 241-262

Orman G, Benson JE, Kweldam CF, Bosemani T, Tekes A, de Jong MR, Seyfert D, Northington FJ, Poretti A, Huisman TAGM (2015): Neonatal head ultrasonography 
today: a powerful imaging tool! J Neuroimaging 25, 31-55

Pan P (2018): Outcome Analysis of Ventriculoperitoneal Shunt Surgery in Pediatric Hydrocephalus. J Pediatr Neurosci 13, 176

Papile L-A, Burstein J, Burstein R, Koffler H, Koops BL, Johnson JD (1980): Posthemorrhagic hydrocephalus in low-birth-weight infants: Treatment by serial lumbar punctures. J Pediatr 97, 273-277

Persson E-K, Anderson S, Wiklund L-M, Uvebrant P (2007): Hydrocephalus in children born in 1999-2002: epidemiology, outcome and ophthalmological findings. Childs Nerv Syst $\underline{23}, 1111-1118$

Phan S, Liao J, Jia F, Maharaj M, Reddy R, Mobbs RJ, Rao PJ, Phan K (2016): Laparotomy vs minimally invasive laparoscopic ventriculoperitoneal shunt placement for hydrocephalus: A systematic review and meta-analysis. Clin Neurol Neurosurg $\underline{140}, 26-$ 32

Pinto-Martin JA, Riolo S (1995): Cranial ultrasound prediction of disabling and nondisabling cerebral palsy at age two in a low birth weight population. Pediatrics 95 , 249-254

du Plessis AJ (2009): The Role of Systemic Hemodynamic Disturbances in PrematurityRelated Brain Injury. J Child Neurol 24, 1127-1140

Poca MA, Martínez-Ricarte FR, Portabella M, Torné R, Fuertes ML, González-Tartiere P, Sahuquillo J (2013): Head circumference: the forgotten tool for hydrocephalus management. A reference interval study in the Spanish population. Clin Neurol Neurosurg $\underline{115}, 2382-2387$

Pollack IF, Albright AL, Adelson PD (1999): A randomized, controlled study of a programmable shunt valve versus a conventional valve for patients with hydrocephalus. Hakim-Medos Investigator Group. Neurosurgery $\underline{45}$, 1399-1411

Popa F, Grigorean VT, Onose G, Popescu M, Strambu V, Sandu AM (2009): Laparoscopic treatment of abdominal complications following ventriculoperitoneal shunt. J Med Life 2, 426-436

Prusseit J, Simon M, von der Brelie C, Heep A, Molitor E, Völz S, Simon A (2009): Epidemiology, Prevention and Management of Ventriculoperitoneal Shunt Infections in Children. Pediatr Neurosurg $\underline{45}, 325-336$

Pumberger W, Löbl M, Geissler W (1998): Appendicitis in children with a ventriculoperitoneal shunt. Pediatr Neurosurg 28, 21-26

Raboei EH (2009): Necrotizing enterocolitis in full-term neonates: is it aganglionosis? Eur J Pediatr Surg $\underline{19}, 101-104$

Rachel RA (1999): Surgical Treatment of Hydrocephalus: A Historical Perspective. Pediatr Neurosurg $\underline{30}, 296-304$ 
Rangel-Castilla L, Barber S, Zhang YJ (2012): The Role of Endoscopic Third Ventriculostomy in the Treatment of Communicating Hydrocephalus. World Neurosurg 77, 555-560

Ransohoff J (1954): Ventriculo-Pleural Anastomosis in Treatment of Midline Obstructional Neoplasms. J Neurosurg $\underline{11}$, 295-298

Ratliff M, Unterberg A, Bächli H (2015): Ventriculo-bipleural shunt as last resort in a 4year-old child in whom a VP and VA shunt failed. J Neurosurg Pediatr 17, 285-288

Rekate HL: Slit Ventricle Syndrome or Syndromes: Diagnosis and Management.; in: Pediatric Hydrocephalus; hrsg. v. Cinalli G, Sainte-Rose C, Maixner WJ; Springer, Mailand 2005, 335-349

Rekate HL, Yonas H, White RJ, Nulsen FE (1979): The acute abdomen in patients with ventriculoperitoneal shunts. Surg Neurol 11, 442-445

De Ribaupierre S, Rilliet B, Vernet O, Regli L, Villemure J-G (2007): Third ventriculostomy vs ventriculoperitoneal shunt in pediatric obstructive hydrocephalus: results from a Swiss series and literature review. Childs Nerv Syst 23, 527-533

Rickham PP (1964): A ventriculostomy reservoir. Br Med J 2, 173

Riva-Cambrin J, Shannon CN, Holubkov R, Whitehead WE, Kulkarni AV, Drake J, Simon TD, Browd SR, Kestle JRW, Wellons JC (2012): Center effect and other factors influencing temporization and shunting of cerebrospinal fluid in preterm infants with intraventricular hemorrhage. J Neurosurg Pediatr $\underline{9}$, 473-481

Riva-Cambrin J, Kestle JRW, Holubkov R, Butler J, Kulkarni AV, Drake J, Whitehead WE, Wellons JC, Shannon CN, Tamber MS, et al. (2015): Risk factors for shunt malfunction in pediatric hydrocephalus: a multicenter prospective cohort study. $\mathrm{J}$ Neurosurg Pediatr 17, 382-390

Robinson JR, Rellinger EJ, Hatch LD, Weitkamp J-H, Speck KE, Danko M, Blakely ML (2017): Surgical necrotizing enterocolitis. Semin Perinatol 41, 70-79

Robinson S (2012): Neonatal posthemorrhagic hydrocephalus from prematurity: pathophysiology and current treatment concepts. J Neurosurg Pediatr $\underline{9}, 242-258$

Rohde V, Behm T, Ludwig H, Wachter D (2011): The role of neuronavigation in intracranial endoscopic procedures. Neurosurg Rev $\underline{35}, 351-358$

Rush DS, Walsh JW, Belin RP, Pulito AR (1985): Ventricular sepsis and abdominally related complications in children with cerebrospinal fluid shunts. Surgery 97, 420-427

Sagan LM, Kojder I, Poncyljusz W (2006): Endoscopic aqueductal stent placement for the treatment of a trapped fourth ventricle. J Neurosurg Pediatr 105, 275-280

Sakka L, Coll G, Chazal J (2011): Anatomy and physiology of cerebrospinal fluid. Eur Ann Otorhinolaryngol Head Neck Dis $\underline{128}$, 309-316 
Sarkiss CA, Sarkar R, Yong W, Lazareff JA (2014): Time dependent pattern of cellular characteristics causing ventriculoperitoneal shunt failure in children. Clin Neurol Neurosurg 127, 30-32

Schirmer M (Hrsg.): Neurochirurgie. 10., neu bearb. und erw. Auflage; Elsevier, Urban und Fischer, München 2005

Schroeder HW, Gaab MR (1999): Endoscopic aqueductoplasty: technique and results. Neurosurgery $\underline{45}, 508-518$

Schuhmann MU, Kural C, Lalla L, Ebner FH, Bock C, Ludwig H-C (2019): Two-Micron Continuous-Wave Laser-Assisted Neuroendoscopy: Clinical Experience of Two Institutions in 524 Procedures. World Neurosurg $\underline{122}$, e81-e88

Schulz M, Bührer C, Pohl-Schickinger A, Haberl H, Thomale U-W (2014): Neuroendoscopic lavage for the treatment of intraventricular hemorrhage and hydrocephalus in neonates. J Neurosurg Pediatr $\underline{13}$, 626-635

Segal MB (1993): Extracellular and cerebrospinal fluids. J Inherit Metab Dis $\underline{16}$, 617638

Serlo W, Fernell E, Heikkinen E, Anderson H, von Wendt L (1990): Functions and complications of shunts in different etiologies of childhood hydrocephalus. Childs Nerv Syst $\underline{6}, 92-94$

Sgouros S, Malluci C, Walsh AR, Hockley AD (1995): Long-term complications of hydrocephalus. Pediatr Neurosurg $\underline{23}, 127-132$

Shannon CN, Acakpo-Satchivi L, Kirby RS, Franklin FA, Wellons JC (2012): Ventriculoperitoneal shunt failure: an institutional review of 2-year survival rates. Childs Nerv Syst 28, 2093-2099

Shannon CN, Carr KR, Tomycz L, Wellons JC, Tulipan N (2013): Time to First Shunt Failure in Pediatric Patients over 1 Year Old: A 10-Year Retrospective Study. Pediatr Neurosurg $\underline{49}, 353-359$

Sharifian A, Abdollahi A, Maddah G, Anaraki F, Alvandipour M, Abbasi Sahebi M, Bigdeli N, Hasanzadeh AM (2013): Spontaneous transanal protrusion of ventriculoperitoneal catheter: a case report. Acta Med Iran 51, 135-138

Shitsama S, Wittayanakorn N, Okechi H, Albright AL (2014): Choroid plexus coagulation in infants with extreme hydrocephalus or hydranencephaly. J Neurosurg Pediatr $\underline{14}, 55-57$

Simon TD, Riva-Cambrin J, Srivastava R, Bratton SL, Dean JM, Kestle JRW (2008): Hospital care for children with hydrocephalus in the United States: utilization, charges, comorbidities, and deaths. J Neurosurg Pediatr $\underline{1}$, 131-137

Singh I, Haris M, Husain M, Husain N, Rastogi M, Gupta RK (2008): Role of endoscopic third ventriculostomy in patients with communicating hydrocephalus: an evaluation by 
MR ventriculography. Neurosurg Rev $\underline{31,} 319$

Sivaganesan A, Krishnamurthy R, Sahni D, Viswanathan C (2012): Neuroimaging of ventriculoperitoneal shunt complications in children. Pediatr Radiol 42, 1029-1046

Smith ER, Butler WE, Barker FG (2004): In-hospital mortality rates after ventriculoperitoneal shunt procedures in the United States, 1998 to 2000: relation to hospital and surgeon volume of care. J Neurosurg Pediatr 100, 90-97

Spader HS, Hertzler DA, Kestle JRW, Riva-Cambrin J (2015): Risk factors for infection and the effect of an institutional shunt protocol on the incidence of ventricular access device infections in preterm infants. J Neurosurg Pediatr 15, 156-160

Spennato P, Cinalli G, Carannante G, Ruggiero C, Del Basso de Caro ML: Multiloculated Hydrocephalus.; in: Pediatric Hydrocephalus; hrsg. v. Cinalli G, Sainte-Rose C, Maixner WJ; Springer, Mailand 2005, 219-244

Stauffer UG, Rickham PP, Soper RT (Hrsg.): Kinderchirurgie: ein kurzgefasstes Lehrbuch. 3., neubearb. und erw. Auflage; Thieme, Stuttgart 1992

Steinbok P, Milner R, Agrawal D, Farace E, Leung GKK, Ng I, Tomita T, Wang E, Wang N, Chu Wong GK, Zhou LF (2010): A Multicenter Multinational Registry for Assessing Ventriculoperitoneal Shunt Infections for Hydrocephalus. Neurosurgery $\underline{67}, 1303-1310$

Stephani U, Harms K, Herting E, Speer CP (1989): Conservative treatment of nonresorptive hydrocephalus in premature infants. Monatsschr Kinderheilkd 137, 218-224

Stoll C, Alembik Y, Dott B, Roth MP (1992): An epidemiologic study of environmental and genetic factors in congenital hydrocephalus. Eur J Epidemiol $\underline{8}, 797-803$

Tamber MS, Klimo P, Mazzola CA, Flannery AM, Pediatric Hydrocephalus Systematic Review and Evidence-Based Guidelines Task Force (2014): Pediatric hydrocephalus: systematic literature review and evidence-based guidelines. Part 8: Management of cerebrospinal fluid shunt infection. J Neurosurg Pediatr 14 Suppl 1, 60-71

Taylor AG, Peter JC (2001): Advantages of delayed VP shunting in post-haemorrhagic hydrocephalus seen in low-birth-weight infants. Childs Nerv Syst 17, 328-333

Trepel M: Neuroanatomie: Struktur und Funktion. 6. Auflage; Urban \& Fischer Verlag/Elsevier, München 2015

Tuli S, Drake J, Lawless J, Wigg M, Lamberti-Pasculli M (2000): Risk factors for repeated cerebrospinal shunt failures in pediatric patients with hydrocephalus. J Neurosurg 92, 31-38

Venes JL (1974): Pleural Fluid Effusion and Eosinophilia Following Ventriculo-pleural Shunting. Dev Med Child Neurol 16, 72-76

Vernet O, Campiche R, de Tribolet N (1995): Long-term results after ventriculo-atrial shunting in children. Childs Nerv Syst 11, 176-179 
Vinchon M, Dhellemmes P: Abdominal Complications of Peritoneal Shunts.; in: Pediatric Hydrocephalus; hrsg. v. Cinalli G, Sainte-Rose C, Maixner WJ; Springer, Mailand 2005, $315-327$

Vinchon M, Baroncini M, Delestret I (2012): Adult outcome of pediatric hydrocephalus. Childs Nerv Syst $\underline{28}, 847-854$

Volpe J (1995): Intracranial hemorrhage: germinal matrix-intraventricular hemorrhage of the premature infant. Neurol Newborn Phila WB Saunders 403-63

Warf BC (2013): Congenital idiopathic hydrocephalus of infancy: the results of treatment by endoscopic third ventriculostomy with or without choroid plexus cauterization and suggestions for how it works. Childs Nerv Syst 29, 935-940

Weinzierl MR, Collmann H, Korinth MC, Gilsbach JM, Rohde V (2007): Management of Hydrocephalus in Children with Plasminogen Deficiency. Eur J Pediatr Surg 17, 124128

West KW, Turner MK, Vane DW, Boaz J, Kalsbeck J, Grosfeld JL (1987): Ventricular gallbladder shunts: an alternative procedure in hydrocephalus. J Pediatr Surg 22, 609612

Whitelaw A, Aquilina K (2012): Management of posthaemorrhagic ventricular dilatation. Arch Dis Child - Fetal Neonatal Ed 97, F229-F233

Willis B, Javalkar V, Vannemreddy P, Caldito G, Matsuyama J, Guthikonda B, Bollam P, Nanda A (2009): Ventricular reservoirs and ventriculoperitoneal shunts for premature infants with posthemorrhagic hydrocephalus: an institutional experience. J Neurosurg Pediatr $\underline{3}, 94-100$

Wilson CB, Bertan V (1966): Perforation of the bowel complicating peritoneal shunt for hydrocephalus. Report of two cases. Am Surg $\underline{32}, 601-603$

Winston KR, Dolan SA (2011): Multidisciplinary approach to cerebrospinal fluid shunt infection with an appeal for attention to details in assessment and standardization in reporting. J Neurosurg Pediatr $\underline{7}, 452-461$

Wollack JB, Makori B, Ahlawat S, Koneru R, Picinich SC, Smith A, Goldman ID, Qiu A, Cole PD, Glod J, Kamen B (2007): Characterization of folate uptake by choroid plexus epithelial cells in a rat primary culture model. J Neurochem 104, 1494-1503 


\section{Danksagung}

Für die freundliche und konstruktive Unterstützung meiner Dissertation bedanke ich mich rechtherzlich bei Prof. Dr. Ludwig, Herrn PD Dr. Grade, Herrn Dr. Bock und Herrn Dr. Kahl. Ich widme diese Arbeit meinen Eltern und Großeltern, die mir stets zur Seite stehen und standen und mich jederzeit mit Herz und Tat auf meinem Weg begleiten. 